SÉRGIO LUIZ BALLIN

CONTROLADOR PREDITIVO MULTIVARIÁVEL COM RESTRIÇÃO DE EXCITAÇÃO PARA IDENTIFICAÇÃO DE PROCESSOS EM MALHA FECHADA 
SÉRGIO LUIZ BALLIN

\section{CONTROLADOR PREDITIVO MULTIVARIÁVEL COM RESTRIÇÃO DE EXCITAÇÃO PARA IDENTIFICAÇÃO DE PROCESSOS EM MALHA FECHADA}

Dissertação apresentada à Escola

Politécnica da Universidade de

São Paulo para obtenção do título de Mestre em Engenharia. 


\title{
CONTROLADOR PREDITIVO MULTIVARIÁVEL COM RESTRIÇÃO DE EXCITAÇÃO PARA IDENTIFICAÇÃO DE PROCESSOS EM MALHA FECHADA
}

\author{
Dissertação apresentada à Escola \\ Politécnica da Universidade de \\ São Paulo para obtenção do título de \\ Mestre em Engenharia. \\ Área de Concentração: \\ Engenharia Química \\ Orientador: \\ Prof. Dr. Darci Odloak
}




\section{FICHA CATALOGRÁFICA}

\section{Ballin, Sérgio Luiz}

Controlador preditivo multivariável com restrição de excitação para identificação de processos em malha fechada $/$ S.L. Ballin. -- São Paulo, 2008.

$128 \mathrm{p}$.

Dissertação (Mestrado) - Escola Politécnica da Universidade de São Paulo. Departamento de Engenharia Química.

1. Identificação (Teoria de sistemas e controle) 2. Controle de processos 3. Controle preditivo I. Universidade de São Paulo. Escola Politécnica. Departamento de Engenharia Química II. t. 


\section{AGRADECIMENTOS}

Ao Prof. Dr. Darci Odloak, por quem já nutria profunda admiração pela inquestionável capacidade e competência e que ao longo destes anos aprendi a respeitar ainda mais como verdadeiro mestre e exemplo a ser seguido. Pela sua dedicação, persistência e paciência o meu mais sincero muito obrigado.

Ao Dr. Oscar Alberto Zanabria Sotomayor, pela cessão dos dados e programas usados em testes comparativos do capítulo 4 deste trabalho e pela franca disponibilidade para discussões e esclarecimentos.

Ao colega de trabalho e amigo Jurg Peter Kurt, primeiro incentivador desta caminhada e fiel defensor nos momentos em que a dedicação a este trabalho conflitava com as demandas profissionais.

Ao Engo. Hélio Toshio Sakurai, gerente de engenharia da PETROBRAS/SIX durante a quase totalidade do tempo em que este trabalho se desenvolveu, pelo necessário estímulo e apoio proporcionados.

À PETROBRAS, pelas incontáveis oportunidades de crescimento e desenvolvimento.

Aos meus pais, pela formação e educação proporcionadas, pelo exemplo e pelo amor incondicional sempre demonstrado.

Às minhas filhas Luiza e Laura, mais que um agradecimento pelo olhar inocente, pelo sorriso sincero ou por simplesmente existirem, um pedido de desculpas pela eventual ausência nos últimos anos.

À minha esposa leda, pelo amor, dedicação, compreensão e paciência, não só no transcurso desta jornada, mas ao longo de todos estes anos de convivência. 


\section{RESUMO}

$\mathrm{Na}$ implementação de controladores MPC, o desenvolvimento e a definição dos modelos do processo é a etapa mais crítica e a que mais consome tempo. Normalmente, os modelos são obtidos através de testes de identificação realizados na planta, onde se observam as respostas em malha aberta das variáveis controladas a perturbações introduzidas individualmente nas variáveis manipuladas. Por este motivo, a aplicação das técnicas de identificação em malha fechada a controladores MPC com restrições nas entradas e/ou saídas é, reconhecidamente, uma área de aplicação de interesse crescente.

Neste trabalho é estudada a modificação do controlador MPC convencional através da inclusão de uma nova restrição de excitação em adição às restrições normais do controlador, com a finalidade de perturbar o processo de forma controlada, propiciando a identificação em malha fechada de modelos mais precisos do processo, a partir de modelos aproximados.

São desenvolvidas quatro abordagens para implementação desta filosofia e apresentadas simulações para vários casos teóricos, utilizando modelos de dois processos industriais obtidos de artigos recentes relacionados a controle multivariável com incertezas nos modelos. Os resultados das simulações indicam que os dados produzidos permitiram a correta identificação dos modelos tanto no caso nominal (modelo igual à planta) quanto para casos onde a planta era diferente do modelo empregado para as predições do MPC.

Palavras-chave: Identificação de sistemas. Identificação em Malha fechada. Controle de processos. Controle preditivo. 


\begin{abstract}
In MPC implementation, the process models development and definition is the most critical and time consuming task. Normally, the models are obtained through plant identification tests where perturbations are individually introduced in the manipulated variable while the controlled variable open-loop behavior is observed. For this reason, the application of closed-loop identification techniques to MPC controllers with input or output constraints is a growing interest area.
\end{abstract}

This work studies the traditional MPC controller modification with the inclusion of a new excitation constraint, in addition to input or output constraints, whose function is to perturb the process in a controlled way, permitting the closed-loop identification of more precise models, based on known approximated models.

Four implementation methodologies are developed and some simulated theoretical cases are presented using models of two industrial processes extracted from recent papers related to multivariable control with models uncertainty. The simulation results show that the obtained datasets allow the identification of the correct model, both in the nominal case (when the model used by MPC is the true model of the plant) and in the uncertain case, where the model used by MPC is different from the true model.

Keywords: System identification. Closed-loop identification. Process control. Predictive control. 


\section{LISTA DE FIGURAS}

Figura 2.1 - O problema de identificação de sistemas.....................................24

Figura 2.2 - Sistema em malha fechada típico .......................................... 30

Figura 2.3 - Métodos tradicionais para excitação externa de um sistema em

malha fechada

Figura 3.1 - Comportamento das variáveis controladas do controlador com

restrição de excitação - Formulação $(P 1)$.

Figura 3.2 - Comportamento das variáveis manipuladas do controlador com

restrição de excitação - Formulação $(P 1)$.

Figura 3.3 - Comportamento das variáveis controladas do controlador com

restrição de excitação - Formulação (P2)

Figura 3.4 - Comportamento das variáveis manipuladas do controlador com

restrição de excitação - Formulação $(P 2)$.

Figura 3.5 - Comportamento das variáveis controladas do controlador com

restrição de excitação - Formulação (P3).

Figura 3.6 - Comportamento das variáveis manipuladas do controlador com

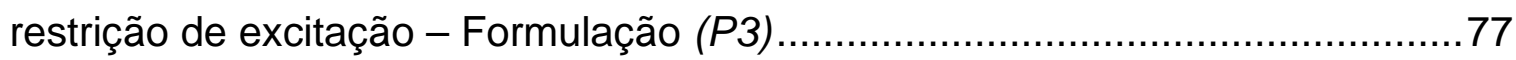

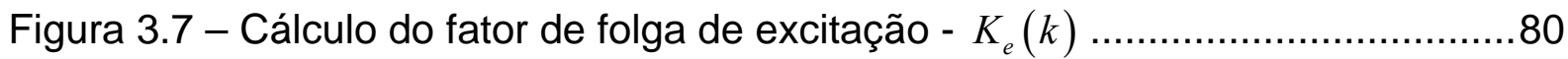

Figura 3.8 - Comportamento das variáveis controladas do controlador com

restrição de excitação - Formulação $(P 4)$.

Figura 3.9 - Comportamento das variáveis manipuladas do controlador com

restrição de excitação - Formulação (P4).

Figura 4.1 - Fluxograma de processo da coluna debutanizadora ........................86

Figura 4.2 - Influência do erro do modelo no desempenho do MPC ....................88

Figura 4.3 - Controlador MPC convencional e com restrição de excitação ..............89

Figura 4.4 - Variáveis controladas da coluna debutanizadora - caso nominal .........91

Figura 4.5 - Variáveis manipuladas da coluna debutanizadora - caso nominal .......91

Figura 4.6 - Validação cruzada do modelo identificado - caso 1 ..........................95

Figura 4.7 - Respostas ao degrau da planta e do modelo identificado - caso 1 ......95

Figura 4.8 - Variáveis controladas da coluna debutanizadora - caso 2 .................96

Figura 4.9 - Variáveis manipuladas da coluna debutanizadora - caso 2 .................97 
Figura 4.10 - Validação cruzada do modelo identificado - caso 2.

Figura 4.11 - Respostas ao degrau da planta, do modelo identificado e do

modelo nominal - caso 2 98

Figura 4.12 - Resposta do controlador MPC sem excitação, com ruído 99

Figura 4.13 - Variáveis controladas da coluna debutanizadora - caso 3 100

Figura 4.14 - Variáveis manipuladas da coluna debutanizadora - caso 3. 100

Figura 4.15 - Respostas ao degrau da planta e do modelo identificado - caso 3

(modelo diferente da planta, com ruído - dados sem tratamento) 101

Figura 4.16 - Validação cruzada do modelo identificado - caso 3 (modelo

diferente da planta, com ruído - dados filtrados) 102

Figura 4.17 - Respostas ao degrau da planta e do modelo identificado - caso 3

(modelo diferente da planta, com ruído - dados filtrados) 102

Figura 4.18 - Variáveis controladas da coluna debutanizadora - caso 4 104

Figura 4.19 - Variáveis manipuladas da coluna debutanizadora - caso 4. 104

Figura 4.20 - Validação cruzada do modelo identificado - caso 4. 105

Figura 4.21 - Respostas ao degrau da planta, do modelo identificado e do

modelo nominal - caso 4 105

Figura 4.22 - Fluxograma de processo simplificado da coluna C3/C4 107

Figura 4.23 - Variáveis controladas da coluna C3/C4 - Caso 5

Figura 4.24 - Variáveis manipuladas da coluna C3/C4 - Caso 5

Figura 4.25 - Validação cruzada dos modelos identificados - Caso 5

Figura 4.26 - Respostas ao degrau dos modelos da coluna C3/C4 - Caso 5.

Figura 4.27 - Respostas ao degrau dos modelos da coluna C3/C4 obtidas nos

trabalhos de Sotomayor; Odloak e Moro (2008). 115

Figura 4.28 - Variáveis controladas da coluna C3/C4 - Caso 6 116

Figura 4.29 - Variáveis manipuladas da coluna C3/C4 - Caso 6 117

Figura 4.30 - Validação cruzada dos modelos identificados - Caso 6 118

Figura 4.31 - Respostas ao degrau dos modelos da coluna C3/C4 - Caso 6 119 


\section{LISTA DE TABELAS}

Tabela 3.1 - Sintonia empregada nos testes do controlador do Problema $(P 1)$.......68

Tabela 3.2 - Sintonia empregada nos testes do controlador do Problema (P2) ......71

Tabela 3.3 - Sintonia empregada nos testes do controlador do Problema (P3) .......76

Tabela 3.4 - Variância dos sinais de entrada e saída para os exemplos das

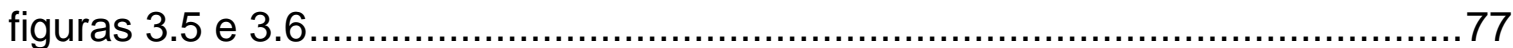

Tabela 3.5 - Sintonia empregada nos testes do controlador do Problema (P4) .......82

Tabela 3.6 - Variância dos sinais de entrada e saída para o controlador (P4) ........84

Tabela 4.1 - Modelos da coluna debutanizadora usados nas simulações...............87

Tabela 4.2 - Sintonia empregada nos testes da figura $4.2 \ldots \ldots \ldots \ldots \ldots \ldots \ldots \ldots \ldots \ldots . \ldots . \ldots . \ldots \ldots$

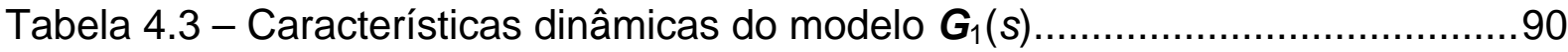

Tabela 4.4 - Busca da ordem do modelo ARX ...............................................92

Tabela 4.5 - Sintonia empregada no caso 5 - controlador $(P 3)$ aplicado à coluna $\mathrm{C} 3 / \mathrm{C} 4$

Tabela 4.6 - Comparação dos índices de ajuste obtidos no teste de validação cruzada para a Coluna C3/C4

Tabela 4.7 - Comparação dos índices de ajuste obtidos no teste de validação cruzada para a Coluna C3/C4 


\section{LISTA DE ABREVIATURAS E SIGLAS}

AIC

ARX

ARMA

ARMAX

CONTSID

DARX

FCC

FIR

GLP

LMI

LTI

LP

MIMO

MISO

MPC

$\mathrm{MPCl}$

OE

OPOM

PE

PEM

Petrobras

PRBS

PVR

QP

RPBC

ROSSMPC

SISO

SQP
Akaike Information Criterion

Auto-Regressive with eXogenous inputs

Auto-Regressive Moving Average

Auto-Regressive Moving Average with eXogenous inputs

Continuous-Time System IDentification

Deterministic ARX

Fluid Catalytic Cracking

Finite Impulse Response

Gás Liquefeito de Petróleo

Linear Matrix Inequalities

Linear, Time Invariant

Linear Programming

Multi-Input, Multi-Output

Multi-Input, Single-Output

Model Predictive Control

Model Predictive Control and Identification

Output Error

Output Prediction Oriented Model

Persistently Exciting

Prediction Error Method

Petróleo Brasileiro S.A.

Pseudo-Random Binary Signal

Pressão de Vapor Reid

Quadratic Programming

Refinaria Presidente Bernardes Cubatão

Reduced Order State-Space MPC

Single-Input, Single-Output

Sequential Quadratic Programming 


\section{LISTA DE SÍMBOLOS}

A matriz característica do sistema

$\boldsymbol{A}_{x} \quad$ matriz característica do sistema no MPC com modelo OPOM

B matriz de distribuição das entradas

$\boldsymbol{B}_{u} \quad$ matriz de distribuição das entradas no MPC com modelo OPOM

C função de transferência do controlador

C matriz das saídas do modelo do sistema

$C_{d} \quad$ matriz dos resíduos dos coeficientes da resposta ao degrau no OPOM

$C_{0} \quad$ matriz dos ganhos em regime permanente no OPOM

c termo independente da função-custo do MPC com modelo OPOM

$\tilde{c}$ termo independente da função-custo do MPC por faixas com modelo OPOM

$c_{f} \quad$ gradiente da função-custo do MPC com modelo OPOM

$\tilde{c}_{f} \quad$ gradiente da função-custo do MPC por faixas com modelo OPOM

D matriz do modelo do OPOM

e vetor de erros

$\overline{\boldsymbol{e}} \quad$ vetor de erros estendido

$\boldsymbol{G}, \boldsymbol{G}_{0}$ função de transferência do processo

$\boldsymbol{H}$ matriz Hessiana da função-custo do MPC com modelo OPOM

$\tilde{\boldsymbol{H}}$ matriz Hessiana da função-custo do MPC por faixas com modelo OPOM

$\boldsymbol{H}_{0} \quad$ função de transferência do modelo do ruído

$\overline{\mathbf{I}} \quad$ matriz auxiliar no MPC com modelo OPOM

$\boldsymbol{J}_{i} \quad$ matriz auxiliar na representação do OPOM

$J_{K} \quad$ função-objetivo ou função-custo

$J_{K 0}$ função-objetivo ou função-custo do MPC sem excitação

$\boldsymbol{K}_{F} \quad$ matriz de ganho do observador de estados

$K_{e} \quad$ fator de folga de excitação no MPC com excitação

$K_{e 1} \quad$ parâmetro de folga para a função-custo do MPC com excitação

$K_{e 2}$ parâmetro de folga para a função-custo do MPC com excitação 
$k \quad$ instante qualquer de tempo discreto

$m \quad$ horizonte de controle

$N \quad$ matriz auxiliar da representação do OPOM

$N \quad$ número de pontos do conjunto de dados

$N \quad$ número de termos do pseudo-vetor de regressores

na ordem da matriz dos coeficientes das saídas da estrutura ARX

$n b \quad$ ordem da matriz dos coeficientes das entradas da estrutura ARX

nd número de estados relacionados aos modos dinâmicos do OPOM

$n k \quad$ ordem da matriz dos coeficientes dos tempos mortos da estrutura ARX

$n p \quad$ horizonte de predição

$n r \quad$ horizonte de predição mínimo

nu número de entradas do sistema

$n x \quad$ número de estados do sistema

ny número de saídas do sistema

$p \quad$ número de parâmetros do modelo

$\mathbf{Q} \quad$ matriz de ponderação (pesos) das saídas

$\boldsymbol{Q}_{n p} \quad$ matriz estendida de ponderação (pesos) das saídas

$q_{1} \quad$ peso da excitação no MPC com excitação

$q_{2} \quad$ peso da variável de folga no MPC com excitação

$\boldsymbol{R}$ matriz de ponderação das entradas (fatores de supressão)

$\boldsymbol{R}_{m} \quad$ matriz estendida de ponderação das entradas (fatores de supressão)

$\boldsymbol{S}_{i} \quad$ matriz dos coeficientes da resposta ao degrau

$S_{0} \quad$ função sensibilidade

$s \quad$ coeficiente da resposta ao degrau

$T$ período de amostragem

u variáveis manipuladas (entradas) do sistema

$\Delta \boldsymbol{u}(k)$ vetor das ações de controle no instante $k$

$\Delta \boldsymbol{u}_{k} \quad$ vetor das ações de controle ao longo do horizonte de controle $m$, calculadas no instante $k$

$\Delta \tilde{\boldsymbol{u}} \quad$ vetor aumentado das ações de controle no MPC por faixas

V função-perda 
$\boldsymbol{v} \quad$ vetor de correção dos estados com as medidas da planta

$\boldsymbol{x} \quad$ vetor de estados do sistema

$\boldsymbol{x}^{s} \quad$ vetor de estados do OPOM relacionados à saída no regime permanente

$\boldsymbol{x}^{d} \quad$ vetor de estados do OPOM relacionados aos modos dinâmicos do modelo

y variáveis controladas (saídas) do sistema

$\bar{y} \quad$ vetor estendido das saídas do sistema

$\bar{y} \quad$ valor médio das saídas medidas

$\hat{y} \quad$ saídas estimadas do modelo

$\boldsymbol{y}^{s p} \quad$ sinal de referência ("set-points") das variáveis controladas do sistema

\section{Símbolos Gregos}

$\varepsilon \quad$ erro de predição do modelo

$\theta \quad$ vetor de parâmetros do modelo

$\bar{\theta} \quad$ estimativa do vetor de parâmetros do modelo

$\theta_{i, j} \quad$ tempo morto entre a j-ésima entrada e a i-ésima saída do sistema

$\lambda$ fator de esquecimento no $\mathrm{MPCl}$

$\mu \quad$ variável de folga no MPC com excitação

$\rho$ fator de excitação no MPC com excitação

$\rho_{0} \quad$ nível mínimo de excitação no MPC com excitação

$\Phi$ matriz de informação do sistema

$\Phi_{i} \quad$ matriz auxiliar com os pólos do modelo no OPOM

$\bar{\Phi}_{i} \quad$ matriz auxiliar com os pólos do modelo no OPOM com tempo morto

$\varphi \quad$ vetor de regressores do modelo

$\Psi \quad$ pseudo-matriz de informação do sistema

$\Psi \quad$ matriz com os pólos do modelo no OPOM

$\bar{\Psi} \quad$ matriz com os pólos do modelo no OPOM com tempo morto

$\psi \quad$ pseudo-vetor de regressores do modelo 
Sobrescritos

$T$ indica a operação de transposição de uma matriz 


\section{SUMÁRIO}

1 INTRODUÇÃO

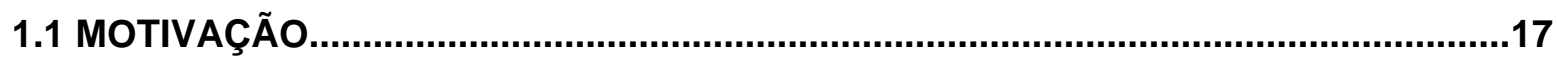

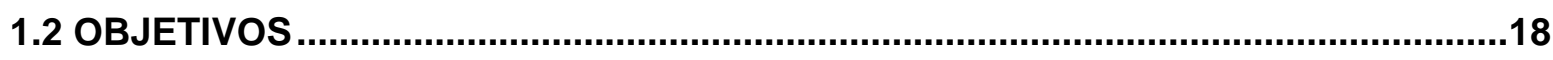

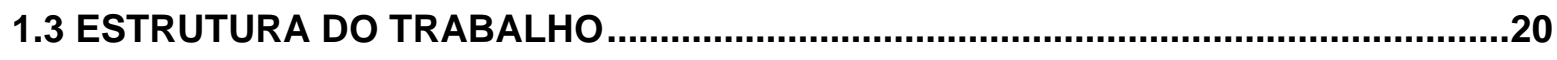

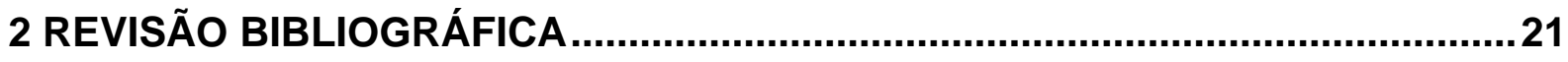

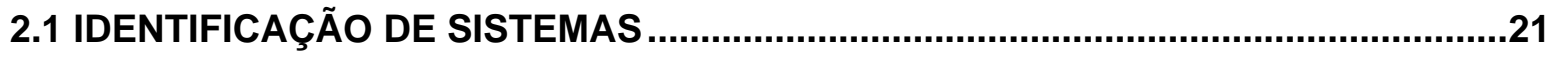

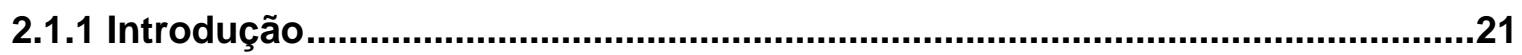

2.1.2 O problema de identificação de sistemas .........................................................22

2.1.3 Projeto do experimento de identificação..........................................................24

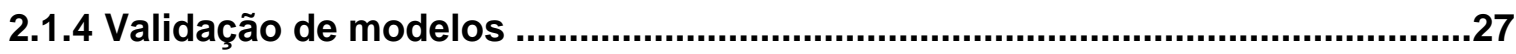

2.1.5 Identificação em malha fechada ..................................................................30

2.2 CONTROLE PREDITIVO BASEADO EM MODELO - MPC ........................................34

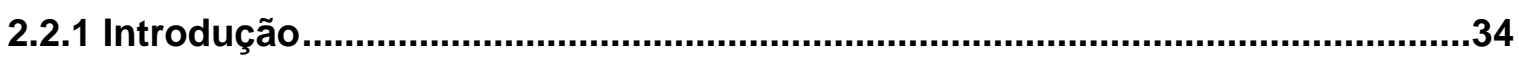

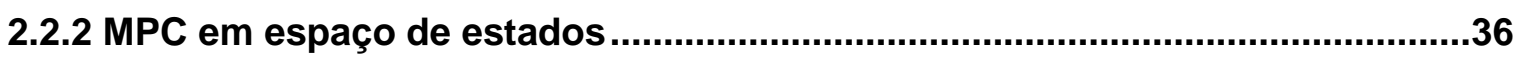

2.2.3 Modelo Orientado à Predição de Saída - OPOM ................................................37

2.2.4 MPC com modelo no formato OPOM ............................................................49

2.2.5 Incluindo um observador de estados no OPOM ..............................................53

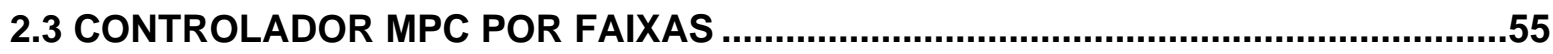

3 CONTROLADOR MPC COM RESTRIÇÃO DE EXCITAÇÃO ...............................57

$3.1 \mathrm{MPCl}$

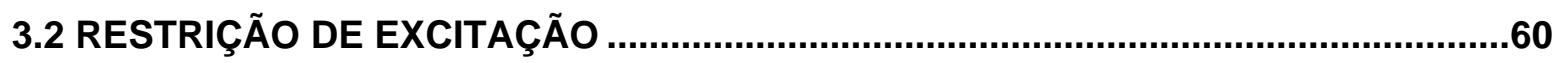

3.3 CONTROLADOR COM MAXIMIZAÇÃO DA EXCITAÇÃO.......................................65

3.4 CONTROLADOR COM EXCITAÇÃO MÍNIMA E VARIÁVEIS DE FOLGA .................70

3.5 CONTROLADOR COM RESTRIÇÃO NO VALOR DA FUNÇÃO-OBJETIVO..............73

3.6 CONTROLADOR POR FAIXAS COM RESTRIÇÃO NO VALOR DA FUNÇÃO-

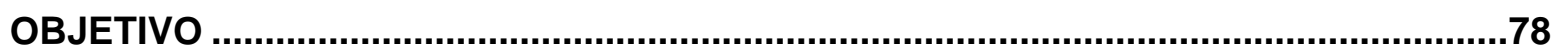

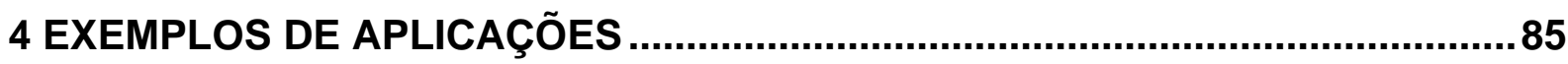

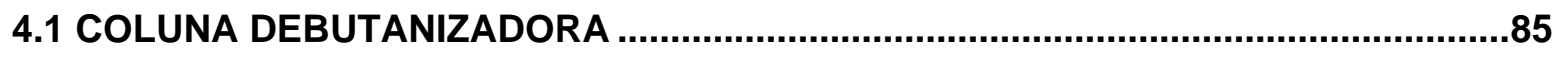

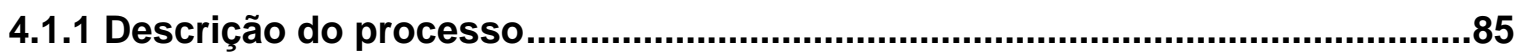

4.1.2 Influência do erro do modelo no desempenho do MPC ....................................87

4.1.3 Controlador com restrição de excitação - caso nominal....................................89

4.1.4 Teste de identificação - caso 1 - caso nominal ..............................................90

4.1.5 Teste de identificação - caso 2 - modelo diferente da planta..........................96 
4.1.6 Teste de identificação - caso 3 - planta com ruído .........................................99

4.1.7 Teste de identificação - caso 4 - controlador por faixas..............................103

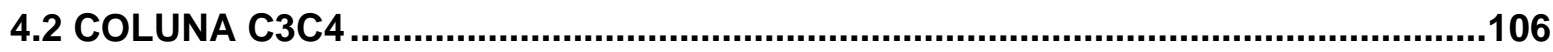

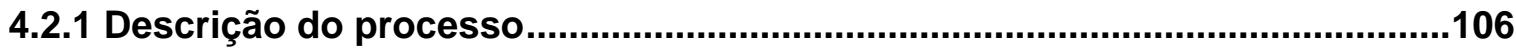

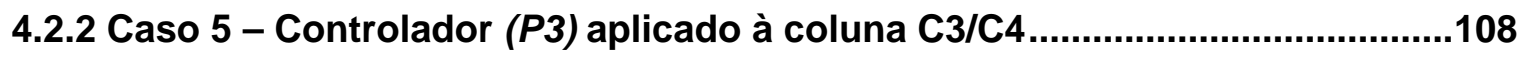

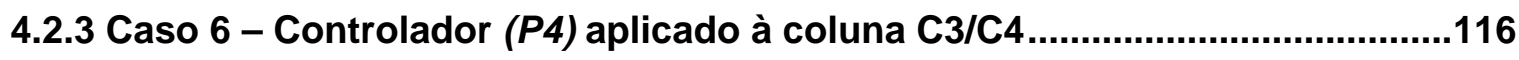

5 RESULTADOS, CONCLUSÕES E SUGESTÕES DE CONTINUIDADE .............122

5.1 CONCLUSÕES .................................................................................................122

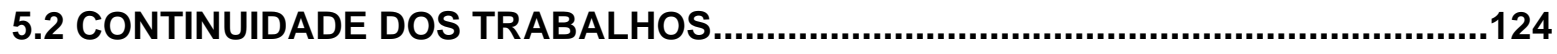

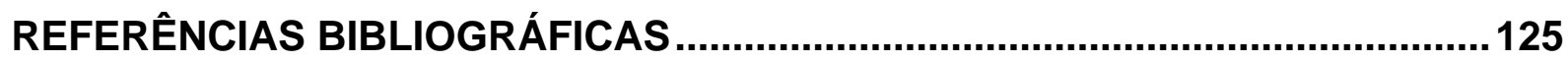




\section{INTRODUÇÃo}

\subsection{MOTIVAÇÃO}

Os controladores preditivos baseados em modelos (MPC, do inglês "Model Predictive Control") pertencem a uma categoria de controladores de comprovada eficiência e aplicação na indústria. Embora tenham sido desenvolvidos para atender às necessidades específicas de plantas de refino de petróleo, têm sido amplamente empregados nos mais diversos processos industriais, principalmente pelas suas características de lidar de forma eficiente com particularidades sempre presentes nestes processos, tais como existência de tempos mortos e restrições nas variáveis manipuladas e controladas, e pela facilidade de se incluir objetivos econômicos diretamente no problema de controle.

Para a implementação dos controladores MPC, o desenvolvimento e a definição dos modelos do processo é seguramente a etapa mais crítica, e a que mais consome tempo. Segundo Rivera; Braun e Mittelmann (2003), estima-se que os esforços gastos na modelagem podem consumir até $75 \%$ do custo de um projeto típico.

A forma mais comum de obtenção dos modelos é através de testes de identificação realizados na planta, onde os engenheiros de controle observam as respostas em malha aberta das variáveis controladas a perturbações introduzidas individualmente nas variáveis manipuladas. Este é um trabalho demorado, de alto custo e que apresenta riscos inerentes de perda de produção ou mesmo de segurança da planta em função da possibilidade das variáveis se afastarem significativamente dos seus parâmetros operacionais.

Ocasionalmente, após algum tempo de operação, os controladores podem apresentar perda de desempenho. Segundo Treiber; Sttelmaier e Starling (2003) após 2 a 3 anos de operação, os controladores raramente apresentam o mesmo desempenho obtido no comissionamento e para o qual foram especificados. Esta 
diminuição na performance do MPC está relacionada principalmente à imprecisão do modelo empregado nas predições em função de mudanças nas características dinâmicas da planta ou da presença de perturbações persistentes que forçam a planta a trabalhar num ponto de operação diferente. Mudanças no comportamento dinâmico da planta podem resultar do desgaste de equipamentos e instrumentos, do decaimento da atividade de catalisadores, ou mesmo de mudanças nas condições de operação ou na especificação dos produtos. Como exemplo de perturbações pode-se citar variações na temperatura ambiente ou na composição da carga. Os ruídos sempre presentes nas medições, nos instrumentos e nos sistemas de transmissão de sinais também podem ser considerados perturbações menores.

Todos estes problemas tendem a se acentuar com o tempo, a ponto de exigir a repetição dos procedimentos de identificação ou mesmo a rotina de identificação periódica dos modelos e ajustes na sintonia do controlador para resgatar os padrões de desempenho aceitáveis originalmente definidos.

Por tudo isto, segundo Forssell e Ljung (1999), a identificação de sistemas em malha fechada é uma área do conhecimento que tem apresentado interesse crescente, tendo como principal incentivo a garantia dos aspectos de segurança e produtividade das plantas (LANDAU - 2001).

A aplicação das técnicas de identificação em malha fechada a controladores MPC com restrições nas entradas e/ou saídas é, portanto, uma área de aplicação de justificado interesse.

\subsection{OBJETIVOS}

Neste trabalho é estudada a modificação do controlador MPC clássico através da inclusão de uma nova restrição, denominada restrição de excitação, em adição às restrições normais do MPC. A finalidade desta nova restrição é de perturbar o processo de forma controlada, propiciando a identificação dos modelos do processo em malha fechada. Esta restrição deve provocar no processo a variabilidade 
suficiente para permitir a identificação dos modelos e ao mesmo tempo garantir os aspectos de segurança da planta e especificação das saídas do controlador.

Utilizam-se como base os estudos apresentados por Genceli e Nikolaou (1996) e Shouche et al. (1998) relativos ao MPC com identificação simultânea (MPCl, do inglês "Model Predictive Control and Identification"), com a diferença de que não se promove nos estudos deste trabalho a adaptação dinâmica do modelo utilizado pelo controlador, ou seja, emprega-se uma filosofia similar para a geração da restrição de excitação, porém, ao contrário do adotado no $\mathrm{MPCl}$, nenhuma atualização é introduzida de forma automática nos modelos empregados pelo MPC. Os dados das variáveis manipuladas e controladas são armazenados e utilizados para identificação "off-line" do processo e os modelos daí obtidos poderão, se desejado, ser reintroduzidos no MPC. Também são estudadas diferentes formas de implementação do novo controlador e da função-objetivo utilizada no mesmo.

Podemos citar ainda os seguintes objetivos complementares deste trabalho:

- Avaliar, através de simulações, o desempenho do novo controlador proposto tanto sob o ponto de vista de controle (manutenção das variáveis controladas dentro de faixas aceitáveis de operação) quanto sob o prisma de identificação em malha fechada (qualidade dos modelos obtidos);

- Comparar os resultados obtidos com outras formas de identificação em malha fechada, preferencialmente com outros métodos de excitação interna;

- Estudar a aplicabilidade prática da metodologia proposta, avaliando aspectos como facilidade de modificação de algoritmos existentes, esforço computacional adicional em comparação ao MPC convencional, estabilidade e funcionalidade do controlador em presença da restrição de excitação proposta, entre outros. 


\subsection{ESTRUTURA DO TRABALHO}

Este trabalho está estruturado em cinco capítulos, incluindo esta introdução.

No capítulo 2 é apresentada a revisão bibliográfica relativa aos assuntos identificação de sistemas e controle preditivo (MPC). No primeiro assunto se procurou enfatizar aspectos importantes relacionados à identificação de sistemas em geral e à identificação em malha fechada em particular; no segundo, se descreve o problema básico do MPC de horizonte finito em espaço de estados, aprofundandose no estudo de Modelos Orientados à Predição da Saída (OPOM), modelagem empregada em todas as formulações de controladores estudados neste trabalho. $\mathrm{O}$ capítulo é concluído com a apresentação de uma nova abordagem para o controle MPC por faixas, base para uma das formulações propostas.

No capítulo 3 é apresentado o MPCl de Genceli e Nikolaou (1996), a restrição de excitação e quatro formulações diferentes testadas neste trabalho para o controlador com restrição de excitação. São apresentados ainda exemplos do comportamento do controlador em cada uma das formulações, simulados com o uso de modelos da indústria de refino de petróleo.

O capítulo 4 descreve os testes de identificação simulados com o uso de modelos de dois processos industriais. São comentados os principais resultados obtidos e os problemas observados.

Finalmente, no capítulo 5 são apresentadas as conclusões deste trabalho e as sugestões para sua futura continuidade. 


\section{REVISÃO BIBLIOGRÁFICA}

Neste capítulo são apresentados e discutidos os principais tópicos relacionados aos temas identificação de sistemas e controle preditivo baseado em modelo (MPC). $\mathrm{Na}$ seção 2.1 são apresentadas definições e discutidas as principais etapas do problema de identificação de sistemas, concluindo com aspectos de identificação em malha fechada. Na seção 2.2 são revistos os conceitos fundamentais do MPC em espaço de estados, passando-se ao detalhamento da modelagem OPOM e ao desenvolvimento do controlador MPC com modelos OPOM. O capítulo é concluído com a apresentação do controlador MPC por faixas na seção 2.3.

\subsection{IDENTIFICAÇÃO DE SISTEMAS}

\subsubsection{Introdução}

Aguirre (2004) define identificação de sistemas como a área do conhecimento que estuda maneiras de modelar e analisar sistemas a partir de observações, ou seja, de dados.

Ljung (1999) explica que a identificação de sistemas trata do problema da construção de modelos matemáticos de sistemas dinâmicos a partir de dados observados.

Em qualquer das definições, entende-se como modelo de um sistema a relação entre os sinais observados. Também é importante ressaltar que um modelo, por mais elaborado que seja, sempre será uma representação imperfeita do sistema real e, como enfatiza Garcia (2005), a aplicação a que se destina determinado modelo é que determinará o seu grau de sofisticação. 
Conforme exposto por Gevers (2006), o desenvolvimento da teoria de identificação foi estimulado pelo desenvolvimento das estratégias de controle baseadas em modelos na década de 60. Os trabalhos de Ho e Kalman (1965, apud GEVERS, 2006) e de Aström e Bohlin (1965, apud GEVERS, 2006) estabeleceram as bases para o desenvolvimento das duas principais técnicas de identificação atualmente empregadas: os métodos de identificação por subespaço de estados e o método do erro de predição (PEM, do inglês "Prediction Error Method"), respectivamente.

Ainda segundo Gevers (2006), um campo que desde os anos 90 tem demonstrado grande desenvolvimento é o de "identificação para controle", um novo paradigma onde a identificação de sistemas é explicitamente vista como uma aproximação, ou seja, a idéia de um modelo exato passa a ser abandonada e se procura controlar o erro do modelo de modo que ele não prejudique o objetivo para o qual está sendo construído ou levantado.

\subsubsection{O problema de identificação de sistemas}

Aguirre (2004) enumera, em linhas gerais, as seguintes etapas envolvidas em um problema de identificação de sistemas:

a) Testes dinâmicos e coleta de dados

b) Escolha da representação matemática a ser usada.

c) Determinação da estrutura do modelo.

d) Estimação de parâmetros.

e) Validação do modelo.

A primeira etapa contempla os procedimentos e critérios para a geração do conjunto de dados a ser utilizado. Nesta etapa estão incluídas a escolha das variáveis, a definição dos sinais de excitação e do período de amostragem e a execução dos testes para coleta dos dados propriamente ditos. A fase de projeto do experimento, inclusa nesta etapa, é considerada por muitos autores o principal fator para determinar o sucesso ou fracasso de um problema de identificação. Para ressaltar 
sua importância, estes autores definem o projeto do experimento como uma etapa a parte, preliminar aos testes e coleta de dados.

Observa-se também que alguns autores condensam as etapas b e c numa única etapa de seleção da estrutura do modelo. Adotando a seqüência proposta por Aguirre anteriormente listada, pode-se exemplificar na segunda etapa a escolha entre modelos contínuos e discretos e na etapa c a definição da ordem e do tempo morto dos modelos. Para o caso de modelos discretos freqüentemente empregados, pode-se incluir ainda a definição da estrutura a ser empregada (FIR, ARX, ARMAX, $\mathrm{OE}$, etc.).

Para a etapa d (estimação de parâmetros) existem diversos algoritmos na literatura, a maioria dos quais são variantes do método clássico de mínimos quadrados.

A última etapa, validação do modelo, consiste em se verificar se os modelos obtidos incorporam ou não as características de interesse do sistema original. Também nesta etapa está a comparação entre possíveis modelos obtidos e a decisão sobre o melhor modelo para a aplicação pretendida. É, portanto, como ressalta Aguirre (2004), uma etapa com elevado grau de subjetividade e que depende muito da quantidade de informação disponível sobre o sistema original.

A figura 2.1, extraída de Garcia (2005), resume as principais fases do problema de identificação de sistemas. 


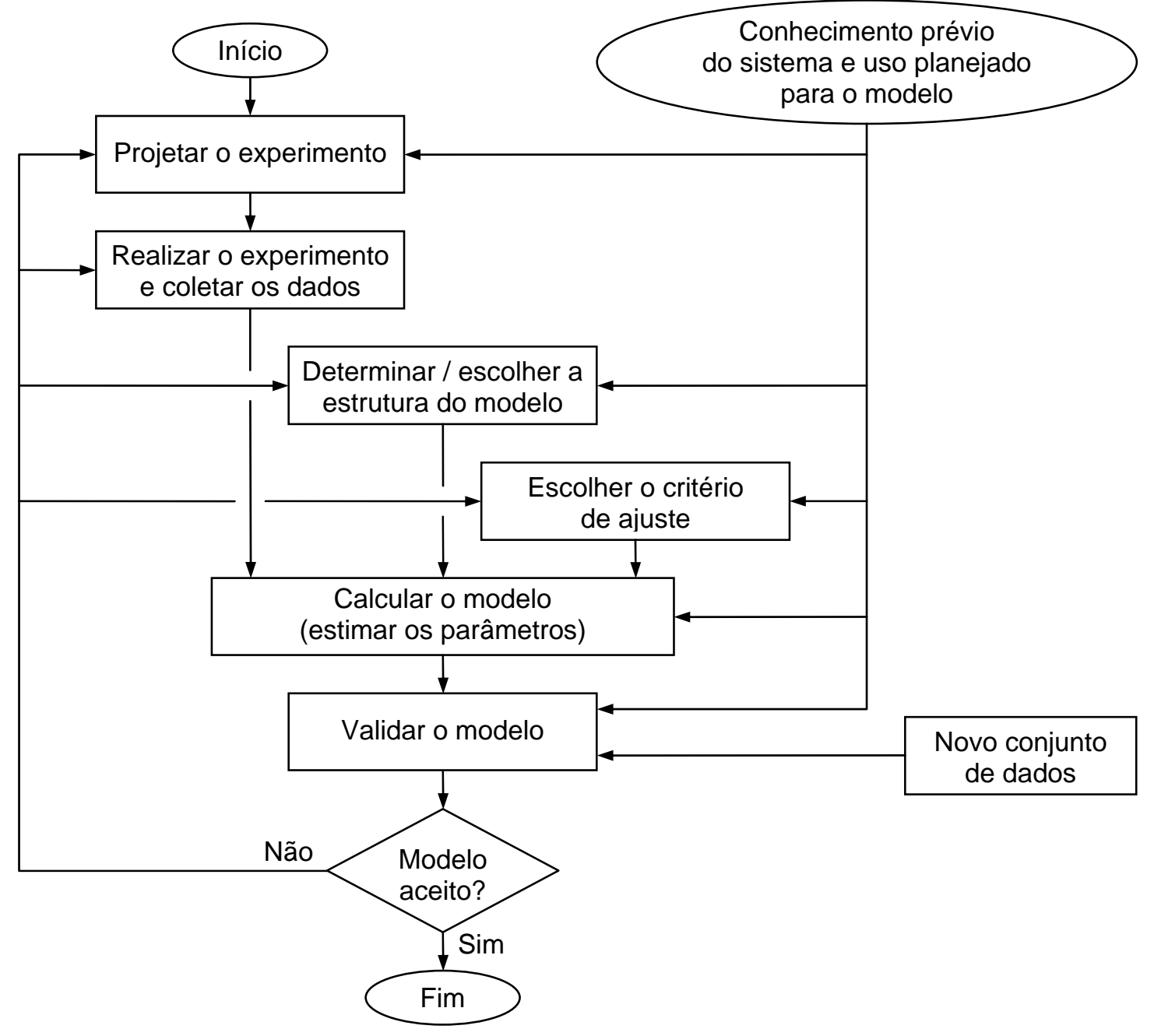

Figura 2.1 - O problema de identificação de sistemas

\subsubsection{Projeto do experimento de identificação}

Nesta seção são apresentados alguns dos mais importantes itens no projeto de um experimento de identificação de sistemas, destacando as escolhas adotadas para os testes que foram executados no capítulo 4 deste trabalho.

a) Período de amostragem

Como se sabe, é sempre pior usar um período de amostragem muito grande do que muito pequeno. Porém, nos testes efetuados neste trabalho, o período de 
amostragem mínimo coincide com o do controlador usado, que já está definido. Resta, portanto, verificar se este período atende às recomendações constantes na literatura.

Duas regras práticas freqüentemente citadas para definir o período de amostragem são: usar um intervalo de amostragem de aproximadamente 1/10 da maior constante de tempo (GUSTAVSSON, 1975); ou 10\% do tempo de acomodação de uma resposta ao degrau.

Uma forma alternativa para determinação do período de amostragem consiste na aquisição de dados na maior freqüência possível ou seja, com períodos de amostragem mais curtos, e utilizar o método heurístico proposto por Aguirre (2004) para definir o período de amostragem final a ser usado na identificação. Este método assume que os sinais foram amostrados com um intervalo muito pequeno e utiliza a análise das autocovariâncias linear e não-linear para definir um fator de decimação a ser empregado na identificação.

b) Tipo de sinal de entrada

Segundo Garcia (2005), a escolha dos sinais de entrada tem influência substancial nos dados observados, uma vez que eles determinarão o ponto de operação do sistema e quais as características dinâmicas e estáticas do sistema que serão excitadas durante o experimento.

Os sinais de entrada mais usados na prática são (SÖDERSTRÖM e STOICA, 1989):

- Degrau;

- PRBS ("Pseudo-Random Binary Signal");

- ARMA ("Auto-Regressive Moving Average”);

- Soma de senóides.

Nas simulações realizadas neste trabalho, como será visto adiante, o sinal de excitação é gerado internamente no controlador MPC, cabendo definir os parâmetros que influenciarão na intensidade da excitação produzida e na ordem (rank) da matriz 
de informação utilizada na restrição de excitação do controlador que, por sua vez, determina a ordem de persistência da excitação.

\section{c) Duração do experimento}

Conforme salienta Garcia (2005), a variância das estimativas é proporcional ao inverso da duração do experimento. Sabendo disso sob a ótica exclusiva da qualidade do modelo identificado, a duração do experimento deveria ser a maior possível. Já em aplicações práticas, sob o ponto de vista operacional, sabendo-se que durante todo o experimento o processo estará sujeito a perturbações extras que podem interferir na operação normal da planta, na qualidade dos produtos e até na segurança do processo, a duração do experimento deveria ser a mínima necessária para a obtenção de um modelo aceitável.

d) Identificação SISO ou MIMO

Na identificação de modelos com apenas uma variável de entrada e de saída (SISO, do inglês "Single-Input, Single-Output") cada par entrada-saída é observado de forma isolada. Quando mais variáveis estão envolvidas, temos modelos multivariáveis, mais adequados na identificação de sistemas onde existem correlações entre variáveis. Estes últimos podem ser divididos em modelos com várias entradas e uma saída (MISO, do inglês "Multi-Input, Single-Output") e modelos com várias entradas e várias saídas (MIMO, do inglês "Multi-Input, MultiOutput"). Nos testes realizados neste trabalho se optou por utilizar a identificação multivariável de modelos ARX ("Auto-Regressive with eXogenous inputs") utilizando o "toolbox" de identificação do programa Matlabß. A identificação MIMO considerada neste aplicativo na verdade consiste de várias identificações MISO simultâneas.

\section{e) Estrutura do modelo}

A escolha da representação e da estrutura do modelo a ser empregada pode envolver decisões como: modelo no domínio do tempo ou da freqüência, em tempo contínuo ou discreto, paramétrico ou não-paramétrico, etc. 
Nas simulações realizadas e descritas no capítulo 4, se optou na maioria das vezes pela utilização de modelos paramétricos multivariáveis com estrutura ARX, por se tratar, segundo Miranda (2005), de uma representação compacta, cujo cálculo é de baixa complexidade e que gera identificação consistente em malha fechada.

\subsubsection{Validação de modelos}

Nesta seção são apresentados os procedimentos de validação e índices/critérios de desempenho de modelos utilizados neste trabalho nos testes do capítulo 4.

\subsubsection{Validação cruzada}

Conforme anteriormente citado, a etapa de validação consiste em se verificar se o modelo obtido incorpora ou não as características de interesse do sistema original. Segundo Garcia (2005), o procedimento conhecido como validação cruzada é um dos mais importantes testes empregado na identificação de sistemas e consiste em se avaliar o desempenho de modelos submetidos a um conjunto de dados para o qual os modelos não foram ajustados, ou seja, um conjunto de dados diferente do utilizado na estimação dos parâmetros, que pode ser um subconjunto de dados do mesmo teste, reservados exclusivamente para validação ou um novo conjunto de dados, oriundo de outro teste.

O desempenho dos modelos a este novo conjunto de dados pode ser medido comparando-se as diferenças entre as saídas reais coletadas da planta e as saídas produzidas pelo modelo. Alguns índices e critérios comumente usados são definidos na próxima seção. 


\subsubsection{Critérios de desempenho}

Um dos mais conhecidos e utilizados critérios para avaliação de modelos é o Critério de Informação de Akaike (AIC, do inglês "Akaike Information Criterion"), definido como (LJUNG, 2007):

$$
A I C=\ln (V)+\frac{2 p}{N}
$$

Onde:

$V \quad$ é a função-perda, definida adiante na eq.(2.2)

$p \quad$ é o número de parâmetros do modelo

$N \quad$ é o número de pontos do conjunto de dados considerado

Como explica Aguirre (2004), o AIC é uma das maneiras usada para estimar a ordem ou o número de parâmetros de um modelo linear. Isto porque, como se sabe, à medida que termos são incluídos no modelo, melhor o ajuste aos dados, com conseqüente redução na variância do erro do modelo. Na eq.(2.1), a primeira parcela quantifica a redução na variância dos resíduos resultante da inclusão de um termo no modelo, ao passo que a segunda parcela penaliza a inclusão de cada termo. Assim, se a redução na primeira parcela da eq.(2.1) devida à inclusão de um novo termo for menor que o custo de incluí-lo, medido pela segunda parcela, o acréscimo deste novo termo não se justifica.

A função-perda $V$ ("loss function") é a função a ser minimizada no método dos mínimos quadrados, definida como (GARCIA, 2005):

$$
V(\boldsymbol{\theta})=\frac{1}{2 N} \sum_{t=1}^{N} \varepsilon^{2}(t, \theta)=\frac{1}{2 N} \sum_{t=1}^{N}[y(t)-\hat{y}(t)]^{2}
$$

\section{Onde}

$\varepsilon \quad$ é o erro de predição do modelo

$\theta \quad$ é o vetor (conjunto) de parâmetros do modelo

$y(t)$ são as saídas observadas/medidas 
$\hat{y}(t) \quad$ são as saídas estimadas do modelo

Outro critério muito utilizado é o erro final de predição (FPE, do inglês "Final Prediction Error"), proposto por Akaike em 1969 e que indica como modificar a função-perda para obter um critério razoável de estimação e validação de modelos com base apenas no conjunto de dados usado na identificação. É definido como (LJUNG, 2007):

$$
F P E=V\left(\frac{1+\frac{p}{N}}{1-\frac{p}{N}}\right)
$$

Onde $V, p$ e $N$ foram anteriormente definidos para a eq.(2.1)

Outros índices eventualmente usados nos gráficos e resultados dos testes de identificação do capítulo 4 são definidos nas eq.(2.4) e (2.5):

$$
\begin{aligned}
& F I T=\left(1-\frac{|y(t)-\hat{y}(t)|}{|y(t)-\bar{y}|}\right) \times 100 \\
& R_{T}^{2}=1-\frac{\operatorname{var}[y(t)-\hat{y}(t)]}{\operatorname{var}[y(t)]}
\end{aligned}
$$

Onde $y(t)$ e $\hat{y}(t)$ foram definidos para a eq.(2.2), enquanto $\bar{y}$ é o valor médio das saídas medidas/observadas. O índice FIT indica o percentual de ajuste dos dados de saída do modelo às saídas medidas. O coeficiente de determinação $R_{T}^{2}$ indica o quão bem a saída do modelo explica o comportamento da saída do sistema e seu valor deve ficar próximo de 1 em condições de baixo ruído (SOTOMAYOR; ODLOAK; MORO, 2008). 


\subsubsection{Identificação em malha fechada}

Diversos fatores podem exigir que os procedimentos de identificação de sistemas sejam conduzidos com o sistema sob controle, ou seja, em malha fechada. Entre estes, Ljung (1999) cita o caso de plantas instáveis ou que devam permanecer sob controle por motivos econômicos, de produção ou de segurança, ou ainda sistemas que possuam mecanismos inerentes de realimentação.

Segundo Ljung (1999), o principal problema da identificação em malha fechada é que tipicamente os dados coletados nesta condição contêm menos informação sobre o sistema que dados coletados em malha aberta. Isto se deve ao fato de que um importante propósito da realimentação é tornar o sistema em malha fechada menos sensível a variações ou perturbações comparado à malha aberta.

Os problemas de identificação em malha fechada consideram processos onde a planta cujo modelo se deseja identificar esteja total ou parcialmente em controle com realimentação, como representado na figura 2.2 .

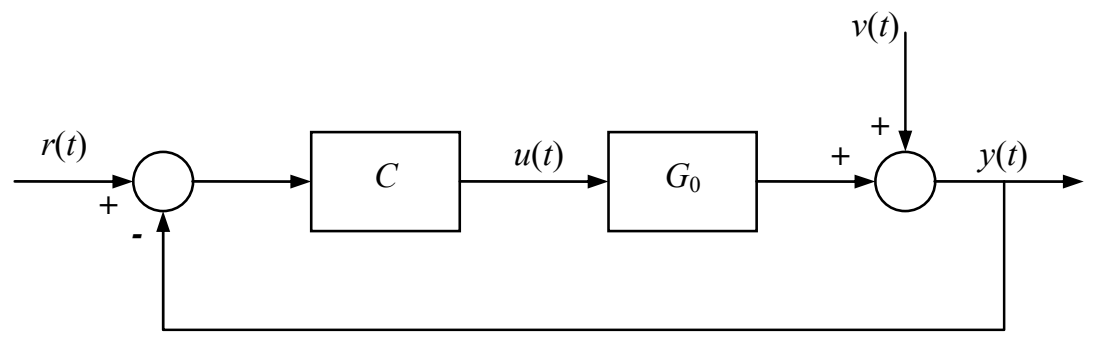

Figura 2.2 - Sistema em malha fechada típico

Nesta figura, $r(t)$ é o set-point, $y(t)$ é a saída do processo, $u(t)$ a entrada, e $v(t)$ é uma perturbação não correlacionada com $r(t)$. $G_{0}$ e $C$ são respectivamente as funções de transferência da planta e do controlador.

A perturbação $v(t)$ pode ser considerada como ruído branco filtrado, ou seja, $v(t)=H_{0} e(t)$, sendo $H_{0}$ o modelo real do erro e $e(t)$ ruído branco com média zero e variância $\lambda_{0}$. 
O sistema real pode ser descrito por:

$$
\begin{aligned}
& y(t)=G_{0} u(t)+v(t)=G_{0} u(t)+H_{0} e(t) \\
& u(t)=C[r(t)-y(t)]
\end{aligned}
$$

As equações em malha fechada são:

$$
\begin{aligned}
& y(t)=G_{0} C S_{0} r(t)+S_{0} v(t) \\
& u(t)=S_{0} C r(t)-C S_{0} v(t)
\end{aligned}
$$

Onde $S_{0}$ é a função sensibilidade, assim definida:

$$
S_{0}=\left(1+C G_{0}\right)^{-1}
$$

Das equações acima, facilmente se observa que a realimentação reduz a influência da perturbação $v(t)$ em malha aberta por um fator correspondente a $S_{0}$.

Entretanto, o propósito da realimentação é tornar a função sensibilidade $S_{0}$ baixa, especialmente nas freqüências onde o sinal de perturbação $v(t)$ tenha energia. Com isto, os dados coletados em malha fechada tornam-se menos informativos sobre o sistema nestas freqüências.

Se por um lado esta característica pode ser considerada um problema, algumas vezes o uso da realimentação pode representar uma vantagem, à medida que o controlador diminui os efeitos das perturbações e mantém o processo dentro dos limites operacionais, resultando em modelos mais confiáveis e com menores incertezas.

Um sistema pode ser identificado se os dados do experimento forem suficientemente informativos e se o conjunto de modelos contiver o sistema real, independente de o sistema estar em malha aberta ou fechada (LJUNG, 1999). 
Segundo Van den Hof (1998), um experimento em malha fechada será informativo em qualquer uma das seguintes situações:

- O sinal injetado é persistentemente excitante (PE - "Persistently Exciting"),

- O controlador é de ordem suficientemente alta, não-linear ou variante no tempo,

- O controlador é "chaveado" entre diferentes sintonias durante o experimento.

As abordagens empregadas para identificação em malha fechada podem ser divididas nas seguintes três categorias (LJUNG, 1999):

a) Método direto ("direct approach"): nesta abordagem, o método do erro de predição (PEM) pode ser empregado diretamente, usando a saída $y(t)$ e a entrada $u(t)$ do processo da mesma forma que em malha aberta, ignorando qualquer possível realimentação e não utilizando o sinal de referência $r(t)$.

b) Método indireto ("indirect approach"): neste caso, o sistema em malha fechada é identificado a partir dos sinais de referência $r(t)$ e de saída $y(t)$. A partir deste sistema em malha fechada e conhecendo-se o controlador, pode-se determinar o modelo da planta.

c) Método conjunto de entrada e saída (“joint input-outut approach”): neste método, a saída $y(t)$ e a entrada $u(t)$ são tratadas como se fossem saídas de um sistema excitado pela referência $r(t)$ e pela perturbação $v(t)$. O modelo da planta pode ser então determinado a partir deste sistema conjunto.

Como se pode ver tanto o método indireto quanto o método conjunto de entrada e saída requerem o conhecimento prévio do controlador e que se assuma que este seja linear e invariante no tempo (LTI, do inglês "Linear, Time Invariant"). Desta forma, nenhum destes dois métodos é adequado para aplicações com controladores MPC, uma vez que o MPC com restrições apresenta características não-lineares e variantes no tempo. 
Assim, o método recomendado para uso no presente trabalho é o método direto, cuja única desvantagem, segundo Forssell e Ljung (1999), é que um modelo adequado do ruído é requerido para prevenir "bias" no modelo estimado da planta.

Segundo Gustavsson; Ljung e Söderström (1977), estudos teóricos mostram que nos problemas de identificação em malha fechada um sinal de excitação externo é necessário para garantir as condições de identificabilidade. A excitação externa deve ser persistentemente excitante (PE) em toda a faixa de freqüências de interesse e deve ser independente de qualquer perturbação do processo.

O sinal externo de excitação pode ser injetado nas variáveis manipuladas $u(t)$, nos "set-points" para as controladas $r(t)$, ou em ambos, como indicado na figura 2.3.

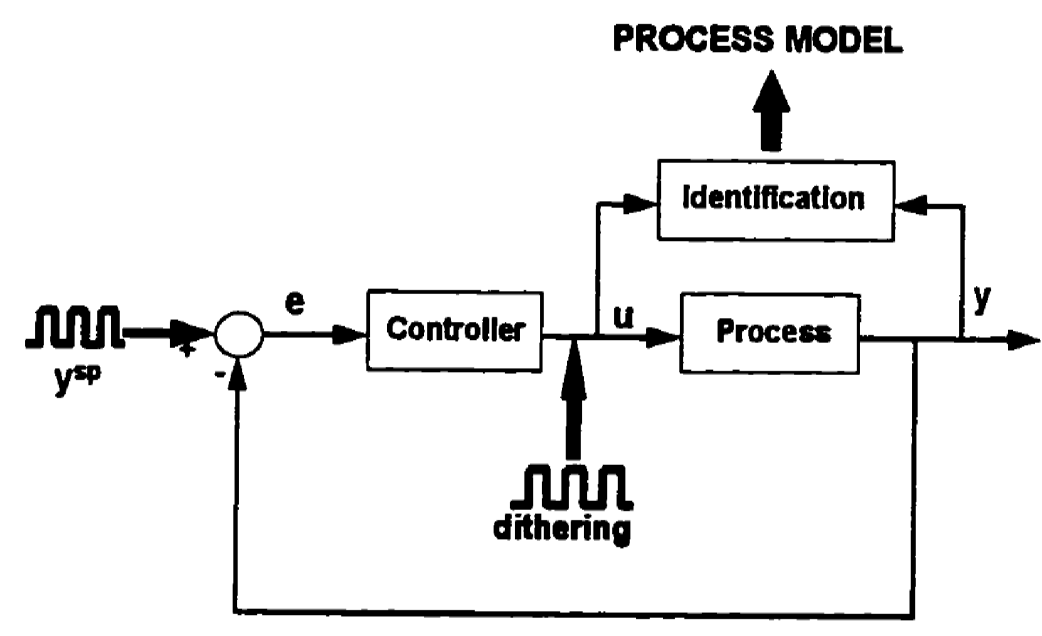

Figura 2.3 - Métodos tradicionais para excitação externa de um sistema em malha fechada

A principal desvantagem desta estratégia é que não há garantia de que as restrições do processo e de especificação dos produtos serão respeitadas durante os testes de identificação. Por outro lado, a utilização de sinais de forma muito conservadora pode resultar em excitação insuficiente, o que pode comprometer os requisitos de identificação. 
Em sistemas de controle com MPC, o sinal de excitação pode ainda ser inserido nas restrições ativas, procedimento adotado no caso industrial apresentado por Miranda (2005).

Em função dos problemas acima descritos, alguns autores propuseram uma nova classe de métodos de excitação para sistemas com MPC que podem ser denominados "métodos com excitação interna". Estes métodos podem ser baseados na modificação da função-custo do controlador de forma a incluir tanto objetivos de controle quanto de identificação, como proposto por Sagias e Nikolaou (2001), ou ainda na inclusão de restrições de excitação, opção usada por Genceli e Nikolaou (1996), Shouche; Genceli e Nikolaou (1998) e Aggeloggianaki e Sarimveis (2006).

A seção 3.1, adiante, descreve esta segunda abordagem com maior profundidade, ao tratar do $\mathrm{MPCl}$ ("Model Predictive Control and Identification").

As formas de excitação adotadas neste trabalho e descritas no capítulo 3 pertencem a esta nova classe de métodos de excitação interna.

\subsection{CONTROLE PREDITIVO BASEADO EM MODELO - MPC}

\subsubsection{Introdução}

O Controle Preditivo baseado em Modelo (MPC, do inglês "Model Predictive Control") pertence a uma classe de algoritmos de controle que utiliza um modelo explícito do processo para estimar a resposta futura da planta. A cada intervalo de tempo, o MPC procura otimizar o comportamento futuro da planta através do cálculo de uma seqüência de ações de controle futuras. A primeira ação de controle é então enviada à planta e a seqüência de cálculos inteira é repetida nos intervalos de tempo subseqüentes. 
Trata-se de uma estratégia de controle na qual as ações de controle são obtidas através da minimização, a cada instante de tempo, de uma função-objetivo (ou função-custo) sujeita a restrições nas amplitudes das entradas e/ou saídas e nas variações das entradas. Na abordagem clássica, o problema de otimização visa minimizar a distância entre a predição da saída e a referência ("set-point") desejada ao longo de um período denominado horizonte de predição. A função-custo normalmente inclui também um termo que penaliza as variações das entradas ao longo de outro período denominado horizonte de controle. As equações abaixo definem o problema de otimização do MPC.

$\min _{\Delta \mathbf{u}_{k}} J_{K}$

Sujeito a

$$
\begin{aligned}
& J_{K}=\sum_{j=1}^{n p}\left[\boldsymbol{y}(k+j)-\boldsymbol{y}^{s p}\right]^{T} \boldsymbol{Q}\left[\boldsymbol{y}(k+j)-\boldsymbol{y}^{s p}\right]+\sum_{j=0}^{m-1} \Delta \boldsymbol{u}(k+j)^{T} \boldsymbol{R} \Delta \boldsymbol{u}(k+j) \\
& -\Delta \boldsymbol{u}^{\max } \leq \Delta \boldsymbol{u}(k+j) \leq \Delta \boldsymbol{u}^{\max } \quad, \quad j=0,1, \ldots, m-1 \\
& \Delta \boldsymbol{u}(k+j)=0, j \geq m \\
& \boldsymbol{u}^{\min } \leq \boldsymbol{u}(k+j) \leq \boldsymbol{u}^{\max } \quad, \quad j=0,1, \ldots, m-1
\end{aligned}
$$

Onde:

$\Delta \boldsymbol{u}_{k}=\left[\begin{array}{llll}\Delta \boldsymbol{u}(k)^{T} & \Delta \boldsymbol{u}(k+1)^{T} & \cdots & \Delta \boldsymbol{u}(k+m-1)^{T}\end{array}\right]^{T}$, calculado no instante $k$

u vetor das entradas manipuladas do sistema

y vetor de saídas controladas do sistema

$\boldsymbol{y}^{s p} \quad$ vetor do sinal de referência ("set-points") das saídas do sistema

$n p \quad$ horizonte de predição

$m \quad$ horizonte de controle

$\mathbf{Q} \quad$ matriz de ponderação (pesos) das saídas

$\boldsymbol{R} \quad$ matriz de ponderação das entradas (fatores de supressão)

$J_{K} \quad$ função-objetivo ou função-custo 


\subsubsection{MPC em espaço de estados}

A grande maioria dos controladores preditivos aplicados em processos industriais utiliza modelos de resposta ao degrau ou de resposta ao impulso finito (FIR, do inglês "Finite Impulse Response") para realizar a predição dos valores das saídas. Já do ponto de vista acadêmico, a maioria dos artigos relacionados ao MPC adotam a representação dos modelos em espaço de estados. Esta representação é bastante conveniente para estudo de sistemas de controle uma vez que as ferramentas matemáticas existentes para análise e síntese de sistemas em malha fechada são normalmente baseadas neste tipo de representação. Em Lee; Morari e Garcia (1994) é mostrada uma interpretação em espaço de estados para o MPC baseado em modelo de resposta ao degrau.

Pode-se utilizar um modelo em espaço de estados resultante da linearização de um modelo fenomenológico ou um modelo correspondente à função de transferência discreta. Neste estudo, considerou-se que o modelo utilizado pelo MPC é descrito pelas seguintes equações:

$$
\begin{aligned}
& \boldsymbol{x}(k+1)=\boldsymbol{A} \boldsymbol{x}(k)+\boldsymbol{B} \Delta \boldsymbol{u}(k) \\
& \boldsymbol{y}(k)=\boldsymbol{C} \boldsymbol{x}(k)
\end{aligned}
$$

Onde $\boldsymbol{x} \in \mathbb{C}^{n x}$ é o vetor de estados, $\boldsymbol{u} \in \mathbb{R}^{n u}$ o vetor de entradas, $\boldsymbol{y} \in \mathbb{R}^{n y}$ o vetor de saídas, $\Delta \boldsymbol{u}(k)=\boldsymbol{u}(k)-\boldsymbol{u}(k-1)$ e $k$ é o instante de tempo atual. $\boldsymbol{A}, \boldsymbol{B}$ e $\boldsymbol{C}$ são matrizes com as dimensões apropriadas. $n x, n u$ e $n y$ são o número de estados, de entradas e de saídas do sistema, respectivamente.

Neste trabalho, conforme se pode observar na eq.(2.16), é empregada a representação incremental nas entradas, o que é adequado para sistemas com perturbações não medidas ou incerteza nos ganhos.

A representação em espaço de estados adotada nos casos estudados neste trabalho empregou a formulação apresentada por Rodrigues e Odloak (2000), 
denominada Modelo Orientado à Predição da Saída (OPOM, do inglês "Output Prediction Oriented Model"), descrita nas seções seguintes.

\subsubsection{Modelo Orientado à Predição de Saída - OPOM}

Nesta seção é apresentado o desenvolvimento do modelo OPOM, conforme proposto por Rodrigues e Odloak (2000). Trata-se de um modelo cujos estados são parâmetros da trajetória de predição da saída, desenvolvido a partir da estratégia originalmente proposta por Tvrzká de Gouvêa e Odloak (1997) sob a designação de ROSSMPC (“Reduced Order State-Space MPC").

\subsubsection{Sistemas SISO}

Considere-se o sistema SISO (uma entrada e uma saída, do inglês "Single-Input, Single-Output") descrito pela seguinte função de transferência:

$$
G(s)=\frac{y(s)}{u(s)}=\frac{b_{0}+b_{1} s+b_{2} s^{2}+\cdots+b_{n b} s^{n b}}{a_{0}+a_{1} s+a_{2} s^{2}+\cdots+s^{n a}}
$$

Onde $\{n a, n b \in \mathbb{N} \mid n b<n a\}$

Admitindo que se trate de um sistema sem tempo morto, não integrador e que possua apenas pólos distintos (não repetidos), pode-se reescrever $G(s)$ fatorando o denominador:

$$
G(s)=\frac{y(s)}{u(s)}=\frac{b_{0}+b_{1} s+b_{2} s^{2}+\cdots+b_{n b} s^{n b}}{\left(s-r_{1}\right)\left(s-r_{2}\right) \cdots\left(s-r_{n a}\right)}
$$

Onde $r_{1}, r_{2}, \ldots, r_{n a}$ são os pólos do sistema, com $r_{i} \neq 0, i=1,2, \ldots, n a$ 
Se a este sistema for aplicado um degrau unitário $u(s)=\frac{1}{s}$, tem-se a seguinte resposta:

$$
y(s)=s(s)=\frac{b_{0}+b_{1} s+b_{2} s^{2}+\cdots+b_{n b} s^{n b}}{s\left(s-r_{1}\right)\left(s-r_{2}\right) \cdots\left(s-r_{n a}\right)}
$$

Expandindo-se em frações parciais e obtendo a transformada inversa de Laplace, chega-se à resposta do sistema no domínio do tempo:

$$
\begin{aligned}
& s(t)=L^{-1}\{s(s)\}=L^{-1}\left\{\frac{c_{0}}{s}+\frac{c_{1}}{s-r_{1}}+\cdots+\frac{c_{n a}}{s-r_{n a}}\right\} \\
& s(t)=c_{0}+c_{1} e^{r_{1} t}+c_{2} e^{r_{2} t}+\cdots+c_{n a} e^{r_{n a} t} \\
& s(t)=c_{0}+\sum_{l=1}^{n a} c_{l} e^{r_{l} t} \quad, \quad t \geq 0
\end{aligned}
$$

Para se entender o conceito do OPOM, suponhamos que no instante atual $(k T)$ a predição para a saída no instante $(k T+t)$, ainda não corrigida com as ações de controle futuras, seja dada pela seguinte trajetória de referência:

$$
y(k+t / k)=p_{0}(k)+p_{1}(k) e^{r_{1} t}+\cdots+p_{n a}(k) e^{r_{n a} t}
$$

Onde $p_{i}(k), i=0,1, \ldots, n a$, são parâmetros da trajetória de referência no instante $(k T)$.

Usando a eq.(2.21) para corrigir esta predição com o efeito da ação de controle $\Delta u(k)$ aplicada ainda no instante $(k T)$, temos:

$$
\begin{aligned}
y^{c}(k+t / k)= & p_{0}(k)+p_{1}(k) e^{r_{1} t}+\cdots+p_{n a}(k) e^{r_{n a} t}+ \\
& +\left[c_{0}+c_{1} e^{r_{1} t}+\cdots+c_{n a} e^{r_{n a} t}\right] \Delta u(k) \\
y^{c}(k+t / k)= & {\left[p_{0}(k)+c_{0} \Delta u(k)\right]+\left[p_{1}(k)+c_{1} \Delta u(k)\right] e^{r_{1} t}+\cdots+} \\
& +\left[p_{n a}(k)+c_{n a} \Delta u(k)\right] e^{r_{n a} t}
\end{aligned}
$$


Aplicando-se a translação para o instante seguinte $(k+1) T$ :

$$
\begin{aligned}
y(k+t / k+1) & =y^{c}(k+t+T / k) \\
y(k+t / k+1) & =\left[p_{0}(k)+c_{0} \Delta u(k)\right]+\left[p_{1}(k)+c_{1} \Delta u(k)\right] e^{r_{1}(t+T)}+\cdots+ \\
& +\left[p_{n a}(k)+c_{n a} \Delta u(k)\right] e^{r_{n a}(t+T)}
\end{aligned}
$$

Pode-se escrever a eq.(2.24) na seguinte forma:

$$
y(k+t / k+1)=p_{0}(k+1)+p_{1}(k+1) e^{r_{1} t}+p_{2}(k+1) e^{r_{2} t}+\cdots+p_{n a}(k+1) e^{r_{n a} t}
$$

Onde:

$$
\begin{aligned}
& p_{0}(k+1)=p_{0}(k)+c_{0} \Delta u(k) \\
& p_{1}(k+1)=p_{1}(k) e^{r_{1} T}+c_{1} e^{r_{1} T} \Delta u(k) \\
& p_{2}(k+1)=p_{2}(k) e^{r_{2} T}+c_{2} e^{r_{2} T} \Delta u(k) \\
& p_{n a}(k+1)=p_{n a}(k) e^{r_{n a} T}+c_{n a} e^{r_{n a} T} \Delta u(k)
\end{aligned}
$$

Ou na equivalente forma matricial;

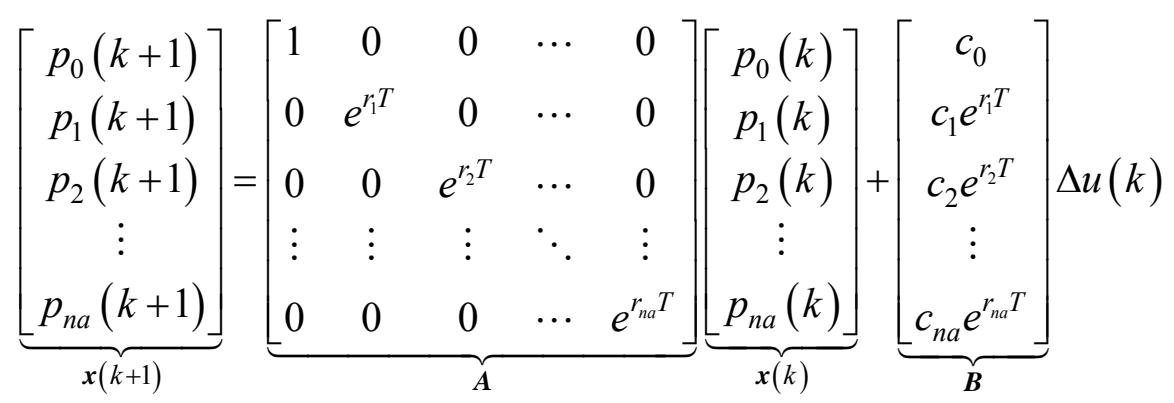

$$
\begin{aligned}
& y(k+t)=\underbrace{\left[\begin{array}{lllll}
1 & e^{r_{1} t} & e^{r_{2} t} & \cdots & e^{r_{n a} t}
\end{array}\right]}_{\boldsymbol{C}} \boldsymbol{x}(k)=\boldsymbol{C} \boldsymbol{x}(k)
\end{aligned}
$$

As duas equações acima já estão no formato apresentado em (2.16) e (2.17). Notese que a saída no instante $k T$ é:

$$
y(k)=p_{0}(k)+p_{1}(k)+p_{2}(k)+\cdots+p_{n a}(k)=\underbrace{\left[\begin{array}{llll}
1 & 1 & \cdots & 1
\end{array}\right]}_{C} x(k)
$$


Assim, de (2.26) e (2.28):

$$
\begin{aligned}
& \boldsymbol{x}(k+1)=\boldsymbol{A} \boldsymbol{x}(k)+\boldsymbol{B} \Delta \boldsymbol{u}(k) \\
& y(k)=\boldsymbol{C} \boldsymbol{x}(k)
\end{aligned}
$$

Onde $A, B$ e $C$ estão indicados nas eq.(2.26) e (2.28).

Pode-se observar na eq.(2.26) que o vetor de estados $\boldsymbol{x}(k)$ é formado pelos parâmetros da trajetória de referência, que é justamente o conceito da modelagem OPOM.

\subsubsection{Sistemas MIMO}

Um desenvolvimento semelhante pode ser feito para um sistema MIMO (múltiplas entradas e múltiplas saídas). Considere-se o sistema com $n u$ entradas e $n y$ saídas:

$$
\boldsymbol{y}(s)=\boldsymbol{G}(s) \boldsymbol{u}(s) \quad ; \quad \boldsymbol{y} \in \mathbb{R}^{n y} \quad \boldsymbol{u} \in \mathbb{R}^{n u}
$$

Nesta equação, a matriz de funções de transferência é dada por:

$$
\boldsymbol{G}(s)=\left[\begin{array}{cccc}
G_{1,1}(s) & G_{1,2}(s) & \cdots & G_{1, n u}(s) \\
G_{2,1}(s) & G_{2,2}(s) & \cdots & G_{2, n u}(s) \\
\vdots & \vdots & \ddots & \vdots \\
G_{n y, 1}(s) & G_{n y, 2}(s) & \cdots & G_{n y, n u}(s)
\end{array}\right]
$$

Onde:

$$
G_{i, j}(s)=\frac{y(s)}{u(s)}=\frac{b_{i, j, 0}+b_{i, j, 1} s+b_{i, j, 2} s^{2}+\cdots+b_{i, j, n b_{i, j}} s^{n b_{i, j}}}{a_{i, j, 0}+a_{i, j, 1} s+a_{i, j, 2} s^{2}+\cdots+s^{n a_{i, j}}}
$$


$G_{i, j}(s)$ relaciona a $i$-ésima saída à $j$-ésima entrada. Para simplificar a notação, assumiremos que para todo par $(i, j)$ temos $n a_{i, j}=n a$, ou seja, todas as funções de transferências individuais $G_{i, j}(s)$ são consideradas de mesma ordem $n a$.

A resposta ao degrau para cada par $(i, j)$ é dada por:

$$
\begin{aligned}
& s_{i, j}(t)=c_{i, j, 0}+\sum_{l=1}^{n a} c_{i, j, l} l^{r_{i, j,} t} \\
& i=1, \ldots, n y ; \quad j=1, \ldots, n u ; \quad l=1, \ldots, n a
\end{aligned}
$$

Onde:

$c_{i, j, 0} \quad$ é o ganho estático da função de transferência $G_{i, j}(s)$

$c_{i, j, l}$ é o resíduo resultante da expansão em frações parciais da resposta ao degrau de $G_{i, j}(s)$, correspondente à $l$-ésima raiz distinta $\left(r_{i, j, l}\right)$

Para este sistema, a predição da saída $i$ no instante de tempo $t$ será dada por:

$$
y_{i}(k+t)=x_{i}(k)+\sum_{j=1}^{n u} \sum_{l=1}^{n a}\left[x_{(i-1) \cdot n u . n a+(j-1) \cdot n a+n y+l}(k)\right] e^{r_{i, j,} t}, \quad i=1,2, \ldots, n y
$$

A atualização do vetor de estados é dada pelas seguintes equações:

$$
\begin{aligned}
& x_{i}(k+1)=x_{i}(k)+\sum_{j=1}^{n u} c_{i, j, 0} \Delta u_{j}(k) \\
& x_{(i-1) . n u . n a+(j-1) \cdot n a+n y+l}(k+1)=e^{r_{i, j, l} T} x_{(i-1) . n u . n a+(j-1) . n a+n y+l}(k)+c_{i, j, l} l^{r_{i, j,} T} \Delta u_{j}(k) \\
& i=1, \ldots, n y ; \quad j=1, \ldots, n u ; \quad l=1, \ldots, n a
\end{aligned}
$$

Para sistemas estáveis, $\lim _{t \rightarrow \infty} y_{i}(k+t)=x_{i}(k) ; i=1, \ldots, n y$. Assim, é conveniente dividir o vetor de estados em duas partes:

$$
\boldsymbol{x}=\left[\begin{array}{l}
\boldsymbol{x}^{s} \\
\boldsymbol{x}^{d}
\end{array}\right]
$$


Onde:

$$
\begin{aligned}
\boldsymbol{x}^{s} & =\left[\begin{array}{llll}
x_{1} & x_{2} & \cdots & x_{n y}
\end{array}\right]^{T}, \quad \boldsymbol{x}^{s} \in \mathbb{R}^{n y} \\
\boldsymbol{x}^{d} & =\left[\begin{array}{llll}
x_{n y+1} & x_{n y+2} & \cdots & x_{n y+n y . n u . n a}
\end{array}\right]^{T}, \quad \boldsymbol{x}^{d} \in \mathbb{C}^{n d}, \quad n d=n y . n u . n a
\end{aligned}
$$

$\boldsymbol{x}^{s}$ está relacionado ao valor da predição das saídas em regime permanente (parte estática), enquanto $x^{d}$ está associado aos modos dinâmicos do sistema.

Com as definições acima, pode-se reescrever as equações (2.36) e (2.37) na seguinte forma matricial:

$$
\begin{aligned}
& \boldsymbol{x}^{s}(k+1)=\boldsymbol{x}^{s}(k)+\boldsymbol{C}_{0} \Delta \boldsymbol{u}(k) \\
& \boldsymbol{x}^{d}(k+1)=\boldsymbol{D} \boldsymbol{x}^{d}(k)+\boldsymbol{C}_{d} \boldsymbol{D} \boldsymbol{N} \Delta \boldsymbol{u}(k)
\end{aligned}
$$

Onde:

$$
\begin{aligned}
& \boldsymbol{C}_{0}=\left[\begin{array}{ccc}
c_{1,1,0} & \cdots & c_{1, n u, 0} \\
\vdots & \ddots & \vdots \\
c_{n y, 1,0} & \cdots & c_{n y, n u, 0}
\end{array}\right], \boldsymbol{C}_{0} \in \mathbb{R}^{n y \times n u} \\
& \boldsymbol{C}_{d}=\operatorname{diag}\left(c_{1,1,1} \cdots c_{1,1, n a} \cdots c_{1, n u, 1} \cdots c_{1, n u, n a} \cdots c_{n y, 1,1} \cdots c_{n y, 1, n a} \cdots c_{n y, n u, 1} \cdots c_{n y, n u, n a}\right) \quad, \quad \boldsymbol{C}_{d} \in \mathbb{C}^{n d \times n d}
\end{aligned}
$$

$\boldsymbol{D}=\operatorname{diag}\left(e^{r_{1,1,1} T} \cdots e^{r_{1,1, n a} T} \cdots e^{r_{1, n u, 1} T} \cdots e^{r_{1, n u, n a} T} \cdots e^{r_{n y, 1,1} T} \cdots e^{r_{n y, 1, n a} T} \cdots e^{r_{y, n u, 1} T} \cdots e^{r_{n y, n u, n a} T}\right) \quad, \quad \boldsymbol{D} \in \mathbb{C}^{n d \times n d}$

$$
\boldsymbol{N}=\left[\begin{array}{c}
\boldsymbol{J}_{1} \\
\boldsymbol{J}_{2} \\
\vdots \\
\boldsymbol{J}_{n y}
\end{array}\right] \quad, \quad \boldsymbol{N} \in \mathbb{R}^{n d \times n u}
$$




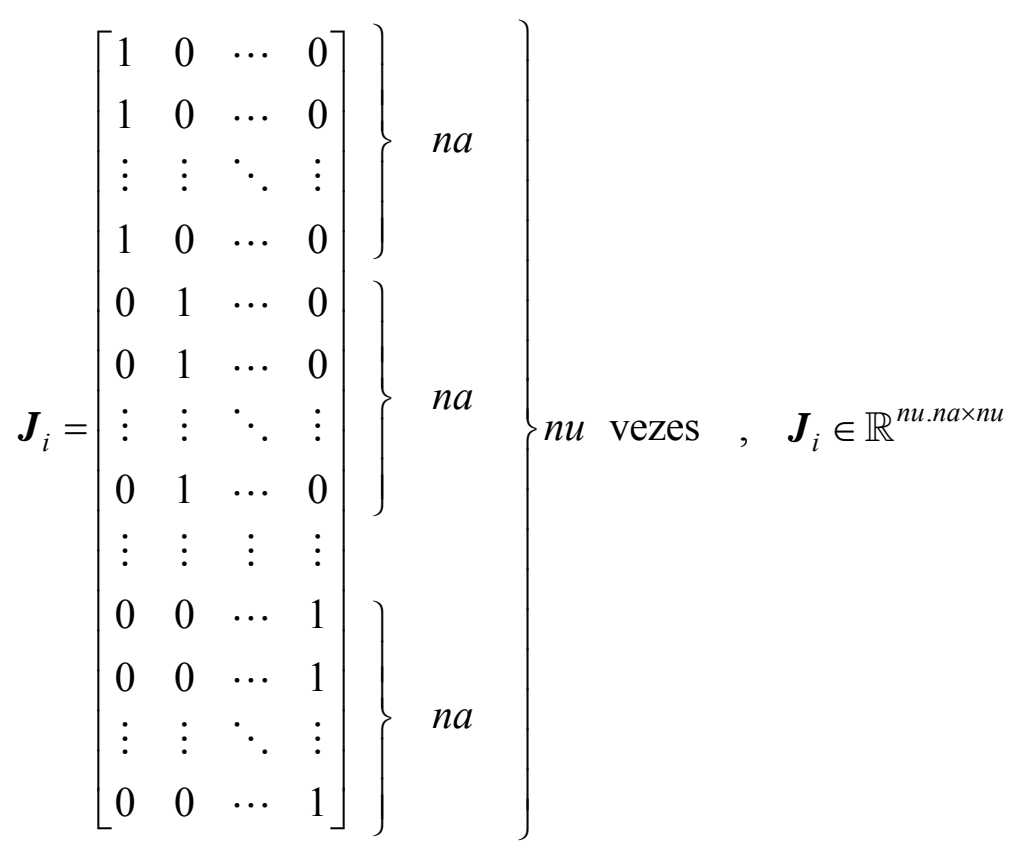

Também a eq.(2.35) pode ser escrita na forma matricial abaixo:

$$
\boldsymbol{y}(k+t)=\boldsymbol{x}^{s}(k)+\Psi(t) \boldsymbol{x}^{d}(k)
$$

Onde:

$\Psi(t)=\left[\begin{array}{cccc}\Phi_{1}(t) & 0 & \cdots & 0 \\ 0 & \Phi_{2}(t) & \cdots & 0 \\ \vdots & \vdots & \ddots & \vdots \\ 0 & 0 & \cdots & \Phi_{n y}(t)\end{array}\right], \quad \Psi(t) \in \mathbb{C}^{n y \times n d}$

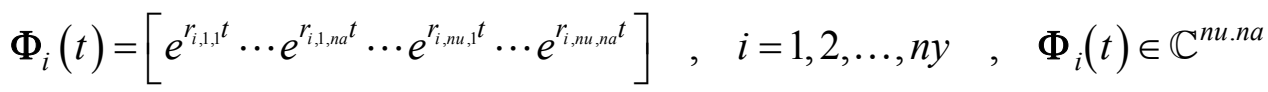

$n d=n y . n u . n a$

Finalmente, as eq.(2.39), (2.40) e (2.46) podem ser colocadas na forma canônica de representação de sistemas em espaço de estados, como apresentado nas eq.(2.16) e (2.17):

$$
\begin{aligned}
& \boldsymbol{x}(k+1)=\boldsymbol{A} \boldsymbol{x}(k)+\boldsymbol{B} \Delta \boldsymbol{u}(k) \\
& \boldsymbol{y}(k+t)=\boldsymbol{C}(t) \boldsymbol{x}(k)
\end{aligned}
$$


Onde:

$\boldsymbol{x}=\left[\begin{array}{l}\boldsymbol{x}^{s} \\ \boldsymbol{x}^{d}\end{array}\right], \boldsymbol{x} \in \mathbb{C}^{n x} ; \quad \boldsymbol{A}=\left[\begin{array}{cc}\boldsymbol{I}_{n y} & \mathbf{0} \\ \mathbf{0} & \boldsymbol{D}\end{array}\right], \boldsymbol{A} \in \mathbb{C}^{n x \times n x} ; \quad \boldsymbol{B}=\left[\begin{array}{c}\boldsymbol{C}_{0} \\ \boldsymbol{C}_{d} \boldsymbol{D N}\end{array}\right], \boldsymbol{B} \in \mathbb{C}^{n x \times n u}$

$\boldsymbol{C}(t)=\left[\begin{array}{ll}\boldsymbol{I}_{n y} & \Psi(t)\end{array}\right], \boldsymbol{C}(t) \in \mathbb{C}^{n y \times n x}$

$n x=n y+n y . n u . n a$

Uma característica importante da modelagem OPOM, que será utilizada adiante no desenvolvimento do controlador MPC, pode ser observada na eq.(2.50), que nos permite calcular a predição da saída em qualquer instante futuro. Caso se tenha interesse no cálculo das saídas apenas no instante atual $(k T)$, para fins de representação na forma usual em espaço de estados, tem-se:

$$
\boldsymbol{y}(k)=\boldsymbol{x}^{s}(k)+\Psi \boldsymbol{x}^{d}(k)
$$

Onde:

$$
\begin{aligned}
& \Psi=\left[\begin{array}{ccc}
\Phi & \cdots & 0 \\
\vdots & \ddots & \vdots \\
0 & \cdots & \Phi
\end{array}\right] \quad, \quad \Psi \in \mathbb{R}^{n y \times n d} \\
& \Phi=\left[\begin{array}{lll}
1 & \cdots & 1
\end{array}\right], \quad \Phi \in \mathbb{R}^{n u . n a}
\end{aligned}
$$

\subsubsection{Sistemas com tempo morto}

Nas duas seções anteriores foi mostrado o desenvolvimento do OPOM para sistemas sem tempo morto. A seguir é apresentada a extensão da abordagem utilizada para o caso com tempo morto.

Considere-se novamente o sistema MIMO apresentado na seção anterior definido pela eq.(2.31), onde a relação entre a $i$-ésima saída e a j-ésima entrada seja dada pela seguinte equação: 


$$
G_{i, j}(s)=\frac{y(s)}{u(s)}=\frac{B_{i, j}(s) e^{-s \theta_{i, j}}}{A_{i, j}(s)}
$$

Onde $\theta_{i, j}$ é o tempo morto entre a $j$-ésima entrada e a $i$-ésima saída e os polinômios $A_{i, j}(s)$ e $B_{i, j}(s)$ são dados por:

$$
\begin{aligned}
& B_{i, j}(s)=b_{i, j, 0}+b_{i, j, 1} s+b_{i, j, 2} s^{2}+\cdots+b_{i, j, n b_{i, j}} s^{n b_{i, j}} \\
& A_{i, j}(s)=a_{i, j, 0}+a_{i, j, 1} s+a i, j,,_{2} s^{2}+\cdots+s^{n a_{i, j}}
\end{aligned}
$$

A resposta ao degrau para cada par $(i, j)$, válida para qualquer instante $n T$, com $n T>$ $\theta_{i, j}$ é dada por:

$$
S_{i, j, n}=\sum_{g=0}^{n a_{i, j}} c_{i, j, g} f_{i, j, g}(n T)
$$

Na eq.(2.53), os termos $c_{i, j, g}$ são os resíduos de $G_{i, j}(s) / s$, enquanto os termos $f_{i, j, g}$, considerando-se apenas sistemas estáveis (não integradores), são dados por:

$$
\begin{aligned}
& \mathrm{p} / g=0: \quad f_{i, j, g}(t)=1 \\
& \mathrm{p} / g \neq 0: \quad f_{i, j, g}(t)=e^{r_{i, j, g}\left(t-\theta_{i, j}\right)}
\end{aligned}
$$

Adotando um desenvolvimento similar ao apresentado para o caso sem tempo morto da seção anterior, podemos assumir que a predição da saída $i$ no instante de tempo $n T$ será dada por:

$$
y_{i}(k+n)=x_{i}(k)+\sum_{j=1}^{n u} \sum_{g=1}^{n a_{i, j}} p_{i, j, g}(k) f_{i, j, g}(n T), \quad i=1,2, \ldots, n y
$$

Onde $x_{i}(k)$ e $p_{i, j, g}(k)$ são, respectivamente, os componentes dos vetores de estado $\boldsymbol{x}^{s}$ e $\boldsymbol{x}^{d}$ anteriormente definidos:

$$
\begin{aligned}
& \boldsymbol{x}^{s}=\left[\begin{array}{llll}
x_{1} & x_{2} & \cdots & x_{n y}
\end{array}\right]^{T}, \quad \boldsymbol{x}^{s} \in \mathbb{R}^{n y} \\
& \boldsymbol{x}^{d}=\left[p_{1,1,1} \cdots p_{1,1, n a_{1,1}} \cdots p_{1, n u, 1} \cdots p_{1, n u, n a_{1, n u}} \cdots p_{n y, 1,1} \cdots p_{n y, 1, n a_{n y, 1}} \cdots p_{n y, n u, 1} \cdots p_{n y, n u, n a_{n y, n u}}\right]^{T}
\end{aligned}
$$


Onde novamente, para simplificar a notação, admitir-se-á que todas as funções de transferências individuais $G_{i, j}(s)$ são de mesma ordem $n a$, ou seja, que $n a_{i, j}=n a$, com $i=1, \ldots, n y$ e $j=1, \ldots, n u$, resultando:

$\boldsymbol{x}^{d}=\left[p_{1,1,1} \cdots p_{1,1, n a} \cdots p_{1, n u, 1} \cdots p_{1, n u, n a} \cdots p_{n y, 1,1} \cdots p_{n y, 1, n a} \cdots p_{n y, n u, 1} \cdots p_{n y, n u, n a}\right]^{T} \quad, \quad \boldsymbol{x}^{d} \in \mathbb{C}^{n d}$

A atualização dos estados é feita da seguinte forma:

$$
\begin{aligned}
& x_{i}(k+1)=x_{i}(k)+\sum_{j=1}^{n u} c_{i, j, 0} \Delta u_{j}(k) \\
& p_{i, j, g}(k+1)=d_{i, j, g} p_{i, j, g}(k)+c_{i, j, l} d_{i, j, g} \Delta u_{j}(k) \\
& i=1, \ldots, n y ; \quad j=1, \ldots, n u ; \quad l=1, \ldots, n a
\end{aligned}
$$

Onde:

$d_{i, j, g}=e^{r_{i, j, l} T} \quad, \quad p / \quad r_{i, j, g} \neq 0$

Pode-se então escrever o modelo em espaço de estados para este sistema:

$$
\begin{aligned}
& \boldsymbol{x}(k+1)=\boldsymbol{A} \boldsymbol{x}(k)+\boldsymbol{B} \Delta \boldsymbol{u}(k) \\
& \boldsymbol{y}(k)=\boldsymbol{C} \boldsymbol{x}(k)
\end{aligned}
$$

Onde:

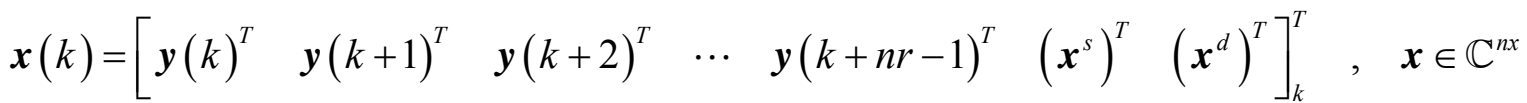

$$
\begin{aligned}
& \boldsymbol{A}=\left[\begin{array}{ccccccc}
0 & \boldsymbol{I}_{n y} & 0 & \cdots & 0 & 0 & 0 \\
0 & 0 & \boldsymbol{I}_{n y} & \cdots & 0 & 0 & 0 \\
\vdots & \vdots & \vdots & \ddots & \vdots & \vdots & \vdots \\
0 & 0 & 0 & \cdots & \boldsymbol{I}_{n y} & 0 & 0 \\
0 & 0 & 0 & \cdots & 0 & \boldsymbol{I}_{n y} & \bar{\Psi}(n r T) \\
0 & 0 & 0 & \cdots & 0 & \boldsymbol{I}_{n y} & 0 \\
0 & 0 & 0 & \cdots & 0 & 0 & \mathbf{D}
\end{array}\right], \quad \boldsymbol{A} \in \mathbb{C}^{n x \times n x}
\end{aligned}
$$




$$
\begin{aligned}
& \boldsymbol{B}=\left[\begin{array}{c}
\boldsymbol{S}_{1} \\
\boldsymbol{S}_{2} \\
\vdots \\
\boldsymbol{S}_{n r-1} \\
\boldsymbol{S}_{n r} \\
\boldsymbol{C}_{0} \\
\boldsymbol{C}_{d} \boldsymbol{D} \boldsymbol{N}
\end{array}\right] \quad, \quad \boldsymbol{B} \in \mathbb{C}^{n \times \times n u} \quad, \quad \boldsymbol{S}_{i}=\left[\begin{array}{cccc}
s_{1,1, i} & s_{1,2, i} & \cdots & s_{1, n u, i} \\
s_{2,1, i} & s_{2,2, i} & \cdots & s_{2, n u, i} \\
\vdots & \vdots & \ddots & \vdots \\
s_{n y, 1, i} & S_{n y, 2, i} & \cdots & s_{n y, n u, i}
\end{array}\right] \\
& \boldsymbol{C}=\left[\begin{array}{lllllll}
\boldsymbol{I}_{n y} & 0 & 0 & \cdots & 0 & 0 & 0
\end{array}\right], \quad \boldsymbol{C} \in \mathbb{R}^{n y \times n x} \\
& n x=(n r+1) n y+n d
\end{aligned}
$$

Com $\mathbf{C}_{0}, \boldsymbol{C}_{d}, \boldsymbol{D}, \boldsymbol{N}$ conforme definido na seção anterior, eq.(2.41), (2.42), (2.43) e (2.44), respectivamente. $\bar{\Psi}(n r T)$ é calculado a partir da eq.(2.47), porém agora considerando os tempos-mortos, conforme abaixo:

$$
\begin{aligned}
& \bar{\Psi}(t)=\left[\begin{array}{cccc}
\bar{\Phi}_{1}(t) & 0 & \cdots & 0 \\
0 & \bar{\Phi}_{2}(t) & \cdots & 0 \\
\vdots & \vdots & \ddots & \vdots \\
0 & 0 & \cdots & \bar{\Phi}_{n y}(t)
\end{array}\right]
\end{aligned}
$$

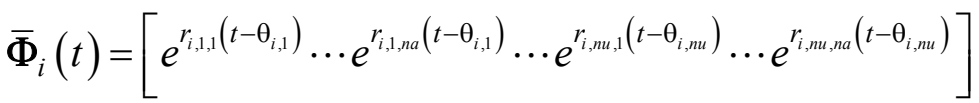

$$
\begin{aligned}
& \bar{\Psi}(n T) \in \mathbb{C}^{n y \times n d} \quad, \quad \bar{\Phi}_{i}(n T) \in \mathbb{C}^{n u . n a}
\end{aligned}
$$

Nas equações anteriores, $n r$ é definido como o horizonte de predição mínimo e deve ser escolhido de tal forma que seja maior que o horizonte de controle $(\mathrm{m})$ adicionado ao maior tempo morto existente no sistema, ou seja:

$$
n r>m+\max _{i, j}\left(\frac{\theta_{i, j}}{T}\right)
$$

Da mesma forma que para o caso sem tempo morto, o uso da modelagem OPOM permite realizar a predição da saída do sistema em qualquer instante futuro $n T$, $n T>n r$, através da eq.(2.62), abaixo:

$$
\boldsymbol{y}(k+n)=\boldsymbol{x}^{s}(k)+\bar{\Psi}(n T) \boldsymbol{x}^{d}(k)
$$


Onde:

$$
\begin{aligned}
& \bar{\Psi}(n T)=\left[\begin{array}{cccc}
\overline{\boldsymbol{\Phi}}_{1}(n T) & 0 & \cdots & 0 \\
0 & \overline{\boldsymbol{\Phi}}_{2}(n T) & \cdots & 0 \\
\vdots & \vdots & \ddots & \vdots \\
0 & 0 & \cdots & \overline{\boldsymbol{\Phi}}_{n y}(n T)
\end{array}\right], \bar{\Psi}(n T) \in \mathbb{C}^{n y \times n d} \\
& \overline{\boldsymbol{\Phi}}_{i}(n T)=\left[e^{r_{i, 1,1}\left(n T-\theta_{i, 1}\right)} \cdots e^{r_{i, 1, n a}\left(n T-\theta_{i, 1}\right)} \cdots e^{r_{i, n u, 1}\left(n T-\theta_{i, n u}\right)} \cdots e^{r_{i, n u, n a}\left(n T-\theta_{i, n u}\right)}\right], \quad \overline{\boldsymbol{\Phi}}_{i}(n T) \in \mathbb{C}^{n u . n a} \\
& i=1,2, \ldots, n y \\
& n d=n y . n u . n a \\
& n x=(n r+1) n y+n d
\end{aligned}
$$

Mais detalhes sobre a formulação OPOM podem ser obtidos nos trabalhos de Odloak (1996), Tvrzká de Gouvêa e Odloak (1997), Rodrigues (2001) e Rodrigues e Odloak (2003). A abordagem para sistemas integradores, não tratada neste trabalho, pode ser encontrada no trabalho de Carrapiço (2004).

\subsubsection{Exemplo de modelagem OPOM}

Nesta seção é apresentado um exemplo da representação OPOM para um sistema simples. Considere-se o seguinte sistema de primeira ordem sem tempo morto, cuja função de transferência é dada por:

$$
G(s)=\frac{K}{\tau s+1}
$$

A resposta deste sistema a uma entrada tipo degrau unitário é dada por:

$$
s(t)=K-K e^{-\frac{t}{\tau}}=c_{0}+c_{1} e^{-\frac{t}{\tau}}
$$

Que pode ser colocada no seguinte modelo de predição orientado à saída (OPOM): 


$$
\begin{aligned}
& \boldsymbol{x}(k+1)=\boldsymbol{A} \boldsymbol{x}(k)+\boldsymbol{B} \Delta \boldsymbol{u}(k) \\
& y(k+t)=\boldsymbol{C}(t) \boldsymbol{x}(k)
\end{aligned}
$$

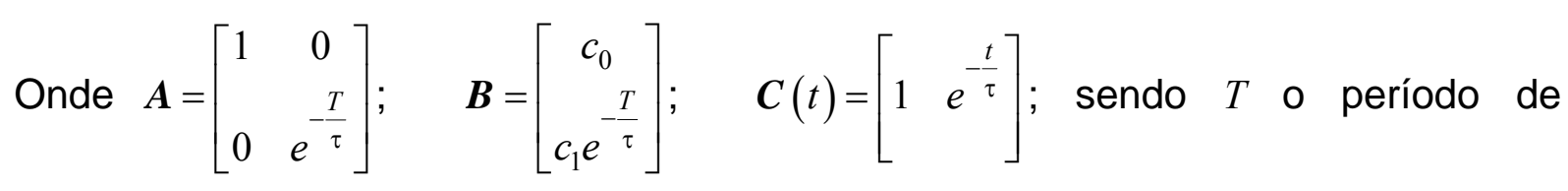
amostragem.

\section{Observações:}

1. Na eq.(2.66) a matriz $C(t)$ não é constante, mas depende do instante de predição $t$ considerado.

2. Se os pólos da função de transferência forem complexos, os coeficientes $c_{i}$ também o serão.

3. No caso de pólos complexos, alguns elementos do vetor de estados também serão complexos.

4. Neste exemplo, um sistema de primeira ordem gera um modelo com dois estados. Pode-se demonstrar facilmente que o número de estados do OPOM para um sistema com ny saídas é igual ao número de componentes do vetor de estados de ordem mínima mais ny.

\subsubsection{MPC com modelo no formato OPOM}

Nesta seção é desenvolvido o problema de otimização para o MPC utilizando a formulação OPOM estudada nas seções anteriores. O problema é tratado com uma formulação geral que engloba tanto o caso particular de sistemas sem tempo morto quanto o caso utilizando a modelagem com tempo morto vista em 2.2.3.3.

Neste desenvolvimento se considerou a formulação de controladores com horizonte de predição $(n p)$ finito. 
Conforme visto na seção 2.2, a função-objetivo (ou função-custo) a ser minimizada pelo MPC tem normalmente a seguinte forma:

$$
J_{K}=\sum_{j=1}^{n p}\left[\boldsymbol{y}(k+j)-\boldsymbol{y}^{s p}\right]^{T} \boldsymbol{Q}\left[\boldsymbol{y}(k+j)-\boldsymbol{y}^{s p}\right]+\sum_{j=0}^{m-1} \Delta \boldsymbol{u}(k+j)^{T} \boldsymbol{R} \Delta \boldsymbol{u}(k+j)
$$

Visando obter uma expressão mais simples do ponto de vista computacional para a função-objetivo definida na eq.(2.67), pode-se usar as eq.(2.57) e (2.58) para avaliar respectivamente os estados e a saída nos instantes de amostragem futuros, conforme detalhado a seguir.

Assim, tem-se:

$$
\begin{aligned}
& \boldsymbol{y}(k+1)=\boldsymbol{C} \boldsymbol{x}(k+1)=\boldsymbol{C A} \boldsymbol{x}(k)+\boldsymbol{C B} \Delta \boldsymbol{u}(k) \\
& \boldsymbol{y}(k+2)=\boldsymbol{C} \boldsymbol{x}(k+2)=\boldsymbol{C A} \boldsymbol{x}(k+1)+\boldsymbol{C} \boldsymbol{B} \Delta \boldsymbol{u}(k+1) \\
& =\boldsymbol{C} \boldsymbol{A}^{2} \boldsymbol{x}(k)+\boldsymbol{C A} \boldsymbol{B} \Delta \boldsymbol{u}(k)+\boldsymbol{C B} \Delta \boldsymbol{u}(k+1) \\
& \quad \vdots \\
& \boldsymbol{y}(k+m)=\boldsymbol{C A}^{m} \boldsymbol{x}(k)+\boldsymbol{C A}^{m-1} \boldsymbol{B} \Delta \boldsymbol{u}(k)+\cdots+\boldsymbol{C B} \Delta \boldsymbol{u}(k+m-1) \\
& \vdots \\
& \quad \vdots \\
& \boldsymbol{y}(k+n p)=\boldsymbol{C A}^{n p} \boldsymbol{x}(k)+\boldsymbol{C A}^{n p-1} \boldsymbol{B} \Delta \boldsymbol{u}(k)+\cdots+\boldsymbol{C A}^{n p-m} \boldsymbol{B} \Delta \boldsymbol{u}(k+m-1)
\end{aligned}
$$

Ou:

$$
\left[\begin{array}{c}
\boldsymbol{y}(k+1) \\
\boldsymbol{y}(k+2) \\
\vdots \\
\boldsymbol{y}(k+m) \\
\vdots \\
\boldsymbol{y}(k+n p)
\end{array}\right]=\left[\begin{array}{c}
\boldsymbol{C A} \\
\boldsymbol{C A}^{2} \\
\vdots \\
\boldsymbol{C A}^{m} \\
\vdots \\
\boldsymbol{C A}^{n p}
\end{array}\right] \boldsymbol{x}(k)+\left[\begin{array}{cccc}
\boldsymbol{C B} & 0 & \cdots & 0 \\
\boldsymbol{C A B} & \boldsymbol{C B} & \cdots & 0 \\
\vdots & \vdots & \ddots & \vdots \\
\boldsymbol{C A}^{m-1} \boldsymbol{B} & \boldsymbol{C A}^{m-2} \boldsymbol{B} & \cdots & \boldsymbol{C B} \\
\vdots & \vdots & \ddots & \vdots \\
\boldsymbol{C A}^{n p-1} \boldsymbol{B} & \boldsymbol{C A}^{n p-2} \boldsymbol{B} & \cdots & \boldsymbol{C A}^{n p-m} \boldsymbol{B}
\end{array}\right]\left[\begin{array}{c}
\Delta \boldsymbol{u}(k) \\
\Delta \boldsymbol{u}(k+1) \\
\vdots \\
\Delta \boldsymbol{u}(k+m-1)
\end{array}\right]
$$

Que em notação vetorial fica:

$$
\overline{\boldsymbol{y}}=\boldsymbol{A}_{x} \boldsymbol{x}(k)+\boldsymbol{B}_{u} \Delta \boldsymbol{u}_{k}
$$

Onde: 
$\overline{\boldsymbol{y}}=\left[\begin{array}{c}\boldsymbol{y}(k+1) \\ \boldsymbol{y}(k+2) \\ \vdots \\ \boldsymbol{y}(k+m) \\ \vdots \\ \boldsymbol{y}(k+n p)\end{array}\right], \quad \overline{\boldsymbol{y}} \in \mathbb{R}^{n y \cdot n p} ; \quad \Delta \boldsymbol{u}_{k}=\left[\begin{array}{c}\Delta \boldsymbol{u}(k) \\ \Delta \boldsymbol{u}(k+1) \\ \vdots \\ \Delta \boldsymbol{u}(k+m-1)\end{array}\right], \quad \Delta \boldsymbol{u}_{k} \in \mathbb{R}^{m \cdot n u}$

$\boldsymbol{A}_{x}=\left[\begin{array}{c}\boldsymbol{C A} \\ \boldsymbol{C A}^{2} \\ \vdots \\ \boldsymbol{C A}^{m} \\ \vdots \\ \boldsymbol{C A}^{n p}\end{array}\right], \quad \boldsymbol{A}_{x} \in \mathbb{C}^{n y \cdot n p \times n x} ; \quad \boldsymbol{B}_{u}=\left[\begin{array}{cccc}\boldsymbol{C B} & 0 & \cdots & 0 \\ \boldsymbol{C A B} & \boldsymbol{C B} & \cdots & 0 \\ \vdots & \vdots & \ddots & \vdots \\ \boldsymbol{C A}^{m-1} \boldsymbol{B} & \boldsymbol{C A}^{m-2} \boldsymbol{B} & \cdots & \boldsymbol{C B} \\ \vdots & \vdots & \ddots & \vdots \\ \boldsymbol{C A}^{n p-1} \boldsymbol{B} & \boldsymbol{C A}^{n p-2} \boldsymbol{B} & \cdots & \boldsymbol{C A}^{n p-m} \boldsymbol{B}\end{array}\right], \quad \boldsymbol{B}_{u} \in \mathbb{C}^{n y \cdot n p \times m \cdot n u}$

Pode-se então definir o seguinte vetor de erros ao longo do horizonte de predição, para utilização na função-custo:

$$
\overline{\boldsymbol{e}}=\overline{\boldsymbol{y}}-\overline{\boldsymbol{y}}^{s p}
$$

Onde:

$$
\begin{aligned}
& \overline{\boldsymbol{y}}^{s p}=\underbrace{\left[\begin{array}{lll}
\left(\boldsymbol{y}^{s p}\right)^{T} & \cdots & \left(\boldsymbol{y}^{s p}\right)^{T}
\end{array}\right]^{T}}_{n p \text { vezes }}=\overline{\boldsymbol{I}} \boldsymbol{y}^{s p} \\
& \left.\overline{\boldsymbol{I}}=\left[\begin{array}{c}
\boldsymbol{I}_{n y} \\
\boldsymbol{I}_{n y} \\
\vdots \\
\boldsymbol{I}_{n y}
\end{array}\right]\right\} n p \quad, \quad \overline{\boldsymbol{I}} \in \mathbb{R}^{n y \cdot n p \times n y}
\end{aligned}
$$

Substituindo a eq.(2.68) na eq.(2.69), tem-se:

$$
\overline{\boldsymbol{e}}=\boldsymbol{A}_{x} \boldsymbol{x}(k)+\boldsymbol{B}_{u} \Delta \boldsymbol{u}_{k}-\overline{\boldsymbol{I}} \boldsymbol{y}^{s p}
$$

Com este resultado, a função-custo definida anteriormente na eq.(2.67) pode ser reescrita na seguinte forma matricial:

$$
J_{K}=\overline{\boldsymbol{e}}^{T} \boldsymbol{Q}_{n p} \overline{\boldsymbol{e}}+\Delta \boldsymbol{u}_{k}^{T} \boldsymbol{R}_{m} \Delta \boldsymbol{u}_{k}
$$




$$
J_{K}=\left[\boldsymbol{A}_{x} \boldsymbol{x}(k)+\boldsymbol{B}_{u} \Delta \boldsymbol{u}_{k}-\overline{\boldsymbol{I}} \boldsymbol{y}^{s p}\right]_{k}^{T} \boldsymbol{Q}_{n p}\left[\boldsymbol{A}_{x} \boldsymbol{x}(k)+\boldsymbol{B}_{u} \Delta \boldsymbol{u}_{k}-\overline{\boldsymbol{I}} \boldsymbol{y}^{s p}\right]_{k}+\Delta \boldsymbol{u}_{k}^{T} \boldsymbol{R}_{m} \Delta \boldsymbol{u}_{k}
$$

Onde:

$\boldsymbol{Q}_{n p}=\operatorname{diag} \underbrace{\left(\begin{array}{lll}\boldsymbol{Q} & \cdots & \boldsymbol{Q}\end{array}\right)}_{n p}$ e $\quad \boldsymbol{R}_{m}=\operatorname{diag} \underbrace{\left(\begin{array}{lll}\boldsymbol{R} & \cdots & \boldsymbol{R}\end{array}\right)}_{m}$

Finalmente, a função-custo $J_{K}$ pode ser colocada na forma abaixo, característica de problemas de programação quadrática:

$$
J_{K}=\Delta \boldsymbol{u}_{k}^{T} \boldsymbol{H} \Delta \boldsymbol{u}_{k}+2 \boldsymbol{c}_{f}^{T} \Delta \boldsymbol{u}_{k}+c
$$

Onde:

$$
\begin{aligned}
& \boldsymbol{H}=\boldsymbol{B}_{u}{ }^{T} \boldsymbol{Q}_{n p} \boldsymbol{B}_{u}+\boldsymbol{R}_{m} \\
& \boldsymbol{c}_{\boldsymbol{f}}{ }^{T}=\left[\boldsymbol{A}_{x} \boldsymbol{x}(k)-\overline{\boldsymbol{I}} \boldsymbol{y}^{s p}\right]^{T} \boldsymbol{Q}_{n p} \boldsymbol{B}_{u} \\
& c=\left[\boldsymbol{A}_{x} \boldsymbol{x}(k)-\overline{\boldsymbol{I}} \boldsymbol{y}^{s p}\right]^{T} \boldsymbol{Q}_{n p}\left[\boldsymbol{A}_{x} \boldsymbol{x}(k)-\overline{\boldsymbol{I}} \boldsymbol{y}^{s p}\right]
\end{aligned}
$$

Pode-se então definir o seguinte problema de otimização para o MPC com modelo OPOM:

Problema (POa)

$$
\min _{\Delta \boldsymbol{u}_{k}} J_{K}=\Delta \boldsymbol{u}_{k}^{T} \boldsymbol{H} \Delta \boldsymbol{u}_{k}+2 \boldsymbol{c}_{f}^{T} \Delta \boldsymbol{u}_{k}+c
$$

Sujeito a

$$
\begin{aligned}
& -\Delta \boldsymbol{u}^{\max } \leq \Delta \boldsymbol{u}(k+j) \leq \Delta \boldsymbol{u}^{\max } \quad, \quad j=0,1, \ldots, m-1 \\
& \Delta \boldsymbol{u}(k+j)=0 \quad, \quad j \geq m \\
& \boldsymbol{u}^{\min } \leq \boldsymbol{u}(k+j) \leq \boldsymbol{u}^{\max } \quad, \quad j=0,1, \ldots, m-1
\end{aligned}
$$

Onde $\boldsymbol{H}, \boldsymbol{c}_{f}$ e $c$ foram definidos nas eq.(2.73) a (2.75) e, 


$$
\boldsymbol{u}(k+j)=\boldsymbol{u}(k-1)+\sum_{i=0}^{j} \Delta \boldsymbol{u}(k+i)
$$

Em algumas formulações do MPC são incluídas também restrições nas variáveis controladas, na seguinte forma:

$$
\boldsymbol{y}^{\min } \leq \boldsymbol{y}(k+j) \leq \boldsymbol{y}^{\max } \quad, \quad j=0,1, \ldots, n p
$$

Deve-se, entretanto, estar atento que este tipo de restrição, embora possível de ser usado na formulação do MPC pode tornar o problema de otimização inviável, motivo pelo qual estas restrições, se presentes, são geralmente não-rígidas.

\subsubsection{Incluindo um observador de estados no OPOM}

No controlador preditivo que emprega a formulação OPOM representada nas eq.(2.49) e (2.50), como em qualquer outra formulação em espaço de estados onde se tenha incerteza no modelo ou a presença de perturbações não medidas, a cada instante de tempo o estado $\boldsymbol{x}(k)$ tem que ser atualizado com a última ação de controle e corrigido com a leitura da saída da planta, o que é feito com o uso de um observador de estados, conforme desenvolvido a seguir.

$$
\begin{aligned}
& \boldsymbol{x}(k+1)=\boldsymbol{A} \boldsymbol{x}(k)+\boldsymbol{B} \Delta \boldsymbol{u}(k) \\
& \boldsymbol{y}(k)=\boldsymbol{C} \boldsymbol{x}(k)
\end{aligned}
$$

Seja a saída real da planta medida no instante de amostragem $(k+1) T$ representada por $\boldsymbol{y}_{p}(k+1)$. Então, o estado do modelo deve ser corrigido com a diferença entre esta medida da planta e a predição dada pelo modelo no instante $(k+1) T$, calculada em $k T, \boldsymbol{y}(k+1 / k)$. Esta diferença pode ser expressa como:

$$
\boldsymbol{v}(k+1)=\boldsymbol{K}_{F}\left[\boldsymbol{y}_{p}(k+1)-\boldsymbol{y}(k+1 / k)\right]
$$


Substituindo as eq. (2.81) e (2.82) em (2.83), tem-se:

$$
\boldsymbol{v}(k+1)=\boldsymbol{K}_{F}\left\{\boldsymbol{y}_{p}(k+1)-\boldsymbol{C}[\boldsymbol{A} \boldsymbol{x}(k / k)+\boldsymbol{B} \Delta \boldsymbol{u}(k)]\right\}
$$

Assim, o estado atualizado com a última ação de controle e corrigido com as medidas da planta fica:

$$
\boldsymbol{x}(k+1 / k+1)=\boldsymbol{A} \boldsymbol{x}(k / k)+\boldsymbol{B} \Delta \boldsymbol{u}(k)+\boldsymbol{v}(k+1)
$$

Substituindo (2.84) em (2.85):

$$
\begin{aligned}
& \boldsymbol{x}(k+1 / k+1)=\boldsymbol{A} \boldsymbol{x}(k / k)+\boldsymbol{B} \Delta \boldsymbol{u}(k)+\boldsymbol{K}_{F} \boldsymbol{y}_{p}(k+1)-\boldsymbol{K}_{F} \boldsymbol{C A} \boldsymbol{x}(k / k)-\boldsymbol{K}_{F} \boldsymbol{C B} \Delta \boldsymbol{u}(k) \\
& \boldsymbol{x}(k+1 / k+1)=\left(\boldsymbol{I}-\boldsymbol{K}_{F} \boldsymbol{C}\right) \boldsymbol{A} \boldsymbol{x}(k / k)+\boldsymbol{K}_{F} \boldsymbol{y}_{p}(k+1)+\left(\boldsymbol{I}-\boldsymbol{K}_{F} \boldsymbol{C}\right) \boldsymbol{B} \Delta \boldsymbol{u}(k)
\end{aligned}
$$

$\boldsymbol{K}_{F}$ é um ganho estático que depende do observador de estados considerado e deve ser selecionado de tal maneira que a matriz $\left(\boldsymbol{I}-\boldsymbol{K}_{F} \boldsymbol{C}\right) \boldsymbol{A}$ seja estável.

Para a formulação do controlador utilizando modelo OPOM sem tempo morto, considerando-se a abordagem usual do DMC, $\boldsymbol{K}_{F}$ tem a seguinte forma:

$$
\boldsymbol{K}_{F}=\left[\begin{array}{ll}
\boldsymbol{I}_{n y} & \boldsymbol{0}_{n y \times n d}
\end{array}\right]^{T}
$$

Já para o modelo OPOM com tempo morto, o observador com a abordagem usual do DMC a ser utilizado é:

$$
\left.\boldsymbol{K}_{F}=\left[\begin{array}{c}
\boldsymbol{I}_{n y} \\
\vdots \\
\boldsymbol{I}_{n y} \\
\boldsymbol{0}_{n d \times n y}
\end{array}\right] n r+1\right]
$$

Deve-se enfatizar que o observador acima só estabiliza $\left(\boldsymbol{I}-\boldsymbol{K}_{F} \boldsymbol{C}\right) \boldsymbol{A}$ quando $\boldsymbol{A}$ tem os autovalores estritamente dentro do círculo unitário. 


\subsection{CONTROLADOR MPC POR FAIXAS}

Algumas implementações de controle MPC empregam uma estratégia na qual as saídas do sistema são controladas de forma a respeitar determinadas faixas ou zonas no lugar de valores de referência ("set-points") fixos (MACIEJOWSKI, 2002). Isto significa que as saídas somente serão tratadas como variáveis controladas quando os valores das predições das mesmas estiverem fora dos limites definidos para as faixas correspondentes.

Usualmente, nesta forma de controle os pesos correspondentes às saídas que estão dentro das respectivas faixas são zerados, de tal forma que estas saídas não são consideradas pelo controlador. Quando a predição da saída ultrapassa os limites definidos para a faixa, o peso correspondente assume o valor de projeto e o "setpoint" é definido igual ao limite da faixa ultrapassado, visando trazer a variável em questão novamente para dentro da faixa.

Nesta seção é desenvolvida uma abordagem alternativa para o MPC por faixas, baseada nos trabalhos de González e Odloak (2008), porém adaptada para os propósitos deste trabalho, ou seja, considerando controlador de horizonte finito, sem a camada de otimização e empregando modelos com a formulação apresentada na seção 2.2.3.3 (OPOM para sistemas com tempo morto).

Nesta abordagem, os "set-points" para as saídas são incluídos no problema de otimização como novas variáveis de decisão. Para minimizar a função-objetivo, os "set-points" podem assumir quaisquer valores dentro das faixas definidas para as saídas. Caso se faça com que os limites superiores e inferiores das faixas sejam idênticos, tem-se o controlador MPC convencional, com "set-points" fixos.

Este novo problema de otimização pode ser então definido como segue:

Problema (POb)

$$
\min _{\Delta \boldsymbol{u}_{k}, \boldsymbol{y}^{s p}} J_{K}
$$


Sujeito a

$$
\begin{aligned}
& J_{K}=\sum_{j=1}^{n p} \boldsymbol{e}(k+j)^{T} \boldsymbol{Q} \boldsymbol{e}(k+j)+\sum_{j=0}^{m-1} \Delta \boldsymbol{u}(k+j)^{T} \boldsymbol{R} \Delta \boldsymbol{u}(k+j) \\
& -\Delta \boldsymbol{u}^{\max } \leq \Delta \boldsymbol{u}(k+j) \leq \Delta \boldsymbol{u}^{\max }, j=0,1, \ldots, m-1 \\
& \Delta \boldsymbol{u}(k+j)=0, j \geq m \\
& \boldsymbol{u}^{\min } \leq \boldsymbol{u}(k+j) \leq \boldsymbol{u}^{\max }, j=0,1, \ldots, m-1 \\
& \boldsymbol{y}^{\min } \leq \boldsymbol{y}^{s p}(k) \leq \boldsymbol{y}^{\max }
\end{aligned}
$$

O desenvolvimento da função-objetivo da eq.(2.90) deve agora ser feito considerando o vetor estendido de variáveis de decisão abaixo indicado.

$$
\Delta \tilde{\boldsymbol{u}}=\left[\begin{array}{ll}
\Delta \boldsymbol{u}_{k}^{T} & \left(\boldsymbol{y}^{s p}\right)^{T}
\end{array}\right]^{T}, \Delta \tilde{\boldsymbol{u}} \in \mathbb{R}^{(m \cdot n u+n y)}
$$

Com esta consideração, pode-se demonstrar que a função-objetivo definida na eq.(2.90) pode ser colocada na seguinte forma:

$$
J_{K}=\Delta \tilde{\boldsymbol{u}}^{T} \tilde{\boldsymbol{H}} \Delta \tilde{\boldsymbol{u}}+2 \tilde{\boldsymbol{c}}_{f}^{T} \Delta \tilde{\boldsymbol{u}}+\tilde{c}
$$

Onde:

$$
\begin{aligned}
& \tilde{\boldsymbol{H}}=\left[\begin{array}{cc}
\boldsymbol{B}_{u}{ }^{T} \boldsymbol{Q}_{n p} \boldsymbol{B}_{u}+\boldsymbol{R}_{m} & -\boldsymbol{B}_{u}{ }^{T} \boldsymbol{Q}_{n p} \overline{\boldsymbol{I}} \\
-\overline{\boldsymbol{I}}^{T} \boldsymbol{Q}_{n p} \boldsymbol{B}_{u} & \overline{\boldsymbol{I}}^{T} \boldsymbol{Q}_{n p} \overline{\boldsymbol{I}}
\end{array}\right] \\
& \tilde{\boldsymbol{c}}_{\boldsymbol{f}}{ }^{T}=\boldsymbol{x}(k)^{T} \boldsymbol{A}_{x}{ }^{T} Q_{n p}\left[\begin{array}{ll}
\boldsymbol{B}_{u} & -\overline{\boldsymbol{I}}
\end{array}\right] \\
& \tilde{\boldsymbol{c}}=\boldsymbol{x}(k)^{T} \boldsymbol{A}_{x}^{T} Q_{n p} \boldsymbol{A}_{x} \boldsymbol{x}(k)
\end{aligned}
$$

Esta formulação foi a base para o controlador que foi empregado nos estudos e simulações da seção 3.6 deste trabalho. 


\section{CONTROLADOR MPC COM RESTRIÇÃO DE EXCITAÇÃO}

Neste capítulo é apresentado o desenvolvimento do controlador MPC com restrição de excitação objeto deste trabalho. Inicialmente, na seção 3.1 é feita a revisão do controle preditivo com identificação simultânea ( $\mathrm{MPCl}$, do inglês "simultaneous constrained Model Predictive Control and Identification"). Em seguida, na seção 3.2 é descrita a formulação da restrição de excitação propriamente dita e, nas seções seguintes, são apresentadas as abordagens utilizadas na formulação do controlador com restrição de excitação. Em todas as abordagens apresentadas para a formulação do controlador se considerou o emprego de modelos de predição orientado à saída (OPOM) anteriormente descritos na seção 2.2.

\section{$3.1 \mathrm{MPCl}$}

Genceli e Nikolaou (1996) propuseram uma nova forma de identificação em malha fechada para sistemas SISO operando com controlador MPC com restrições. Esta técnica, a que se denominou $\mathrm{MPCl}$, visava principalmente aplicação em processos variantes no tempo.

$\mathrm{Na}$ formulação do $\mathrm{MPCl}$, uma entrada para o processo é determinada a cada instante de tempo através da solução de um problema de otimização "on-line" com horizonte móvel ("moving horizon") finito. As entradas devem satisfazer todas as restrições do MPC tradicional, bem como uma restrição adicional que garanta excitação persistente do processo através destas entradas. Através de um algoritmo de identificação baseado na minimização dos erros quadráticos, os parâmetros do modelo são calculados e atualizados a cada instante de tempo, tratando-se, portanto, de um controlador adaptativo.

O MPCI proposto por Genceli e Nikolaou (1996) é formulado a partir de um processo descrito pelo modelo de resposta ao impulso finito (FIR) abaixo. 


$$
y(l / k)=\sum_{i=1}^{n} g_{i}(l / k) u(l-i / k)+d(l / k)
$$

Onde:

$y(l / k)$ saída medida corrente ou passada $(l \leq k)$ ou predição da saída futura $(l>k)$

$u(l / k)$ entrada passada implementada $(l<k)$ ou entrada futura $(l \geq k)$

$g_{i}(l / k)$ estimativa do coeficiente do modelo no instante $l$, feita no instante $k$

$d(l / k)$ estimativa do distúrbio no instante $l$, feita no instante $k$

$n \quad$ ordem do modelo FIR considerado

Que em notação vetorial fica:

$$
y(l / k)=\varphi(l / k)^{T} \theta(l / k)
$$

Onde:

$$
\begin{aligned}
& \varphi(l / k)=[u(l-1 / k), u(l-2 / k), \ldots, u(l-n / k), 1]^{T} \\
& \theta(l / k)=\left[g_{1}(l / k), g_{2}(l / k), \ldots, g_{n}(l / k), d(l / k)\right]^{T}
\end{aligned}
$$

O problema de otimização resolvido pelo MPCl é o seguinte:

$$
\min _{u(k / k), \ldots, u(k+m+n-1 / k), \mu} \sum_{i=1}^{n p} w_{i}\left[y(k+i / k)-y^{S P}\right]^{2}+r_{i} \Delta u(k+i-1 / k)^{2}+q \mu^{2}
$$

Sujeito a:

$$
\begin{aligned}
& u^{\min } \leq u(k+i-1 / k) \leq u^{\max } \quad, \quad i=1,2, \ldots, n p \\
& -\Delta u^{\max } \leq \Delta u(k+i-1 / k) \leq \Delta u^{\max } \quad, \quad i=1,2, \ldots, n p \\
& u(k+m+i / k)=u(k+i / k) \quad, \quad i=0,1, \ldots, n-1 \\
& \sum_{j=0}^{m-1} \lambda^{j} \varphi(k-j+i / k) \varphi(k-j+i / k)^{T} \succeq\left(\rho_{0}-\mu\right) \boldsymbol{I} \succ \mathbf{0} \quad, \quad i=1,2, \ldots, n p-1 \\
& y(k+i / k)=\varphi(k+i / k)^{T} \overline{\boldsymbol{\theta}}(k), \quad i=1,2, \ldots, n p
\end{aligned}
$$




$$
\begin{aligned}
& \overline{\boldsymbol{\theta}}(k)=\left[\sum_{j=0}^{m-1} \lambda^{j} \varphi(k-j+i / k) \varphi(k-j+i / k)^{T}\right]^{-1} \times \\
& \times\left[\begin{array}{llll}
\varphi(k / k) & \lambda \varphi(k-1 / k) & \cdots & \lambda^{m-1} \varphi(k-m+1 / k)
\end{array}\right] \boldsymbol{y}(k) \quad, \quad i=1,2, \ldots, n p
\end{aligned}
$$

Onde:

$$
\begin{aligned}
& \Delta u(k+i-1 / k)=u(k+i-1 / k)-u(k+i-2 / k) \\
& y(k)=[y(k), y(k-1), \ldots, y(k-m+1)]^{T} \\
& n p=m+n
\end{aligned}
$$

Nesta formulação pode-se fazer as seguintes observações:

- A função-objetivo definida na eq.(3.3) é a mesma do MPC convencional acrescida do último termo, que penaliza a variável $\mu$. Esta é uma variável de relaxação incluída para que a restrição da eq.(3.7) não torne o problema inviável. $\mathrm{Na}$ condição ideal para fins de identificação, com máxima excitação, $\mu$ deve ser igual a zero. Também pode ser observado que neste controlador o horizonte de predição $(n p)$ é definido como igual à soma do horizonte de controle $(m)$ com a ordem do modelo FIR considerado $(n)$.

- A restrição da eq.(3.7) é a restrição de excitação. Nesta, o termo $\rho_{0}$ é um parâmetro de sintonia do MPCl que define o limite inferior de excitação.

- A eq.(3.8) é a equação do modelo do processo, utilizada nas predições do controlador. Os coeficientes do modelo $(\bar{\theta})$ são assumidos constantes durante o horizonte de controle e são calculados "on-line" através da eq.(3.9).

- O fator $\lambda,(0<\lambda \leq 1)$ presente nas eq.(3.7) e (3.9) é um fator de esquecimento, cujo propósito é de gradualmente reduzir a importância dos dados mais antigos na restrição de excitação e no cálculo dos coeficientes do modelo. 
Ainda com relação à restrição de excitação dada pela eq.(3.7), cabe observar que a mesma é uma restrição não-convexa, devido à direção da inequação e ao fato de que os termos das matrizes são funções quadráticas das entradas (SHOUCHE; GENCELI; NIKOLAOU, 2002). Além disso, por ser quadrática nas variáveis de entrada do processo, faz com que o MPCI não seja um problema de otimização passível de resolução com algoritmos de programação linear (LP) ou quadrática (QP) como o MPC normal. A solução adotada por Genceli e Nikolaou (1996) foi substituir esta restrição por uma aproximação linear da mesma na forma de desigualdades matriciais lineares (LMI, do inglês "Linear Matrix Inequalities").

Como este problema envolve o cálculo do modelo a cada instante de tempo, é definido um procedimento de inicialização que garante a persistência da excitação definida pela linearização da eq.(3.7) ao longo de todos os instantes do horizonte de predição, inclusive em relação a entradas já implementadas (passadas).

Outras abordagens para o $\mathrm{MPCl}$ podem ser encontradas na literatura, como a extensão da formulação acima para processos descritos por modelos DARX ("Deterministic ARX") apresentada por Shouche et al. (1998) e as variantes propostas por Eker e Nikolaou (1999), todas para sistemas SISO.

Uma abordagem para sistemas MIMO, utilizando otimização multi-objetivo, pode ser encontrada no trabalho de Aggelogiannaki e Sarimveis (2006).

\subsection{RESTRIÇÃO DE EXCITAÇÃO}

Para apresentar a restrição de excitação empregada neste trabalho é conveniente iniciar com o detalhamento da restrição de excitação empregada no $\mathrm{MPCl}$ de Genceli e Nikolaou (1996) visto na seção anterior. Nesta formulação é abordado o caso SISO e o modelo do processo considerado está na forma de resposta ao impulso finito, conforme a eq.(3.10), a seguir. 


$$
y(k)=\sum_{i=1}^{n} g_{i} u(k-i)+d
$$

Onde $y(k)$ é a saída no instante $k T, u(k-i)$ é o valor da entrada no instante $(k-i) T, g_{i}$ são os coeficientes do modelo, $d$ é uma perturbação constante e $n$ é o número de parâmetros ou ordem do modelo FIR considerado.

Esta mesma equação pode ser reescrita na seguinte forma vetorial:

$$
y(k)=\varphi(k)^{T} \theta
$$

Onde $\theta$ é o vetor de parâmetros a ser identificado e $\varphi(k)$ é vetor de regressores.

$$
\begin{aligned}
& \varphi(k)=[u(k-1), u(k-2), \ldots, u(k-n), 1]^{T} \\
& \boldsymbol{\theta}=\left[g_{1}, g_{2}, \ldots, g_{n}, d\right]^{T}
\end{aligned}
$$

A estimativa $\bar{\theta}$ de $\theta$ pode ser obtida pelo método de minimização do erro quadrático ("least squares error minimization"). Uma solução única é garantida se a matriz de informação $\Phi$ indicada abaixo na eq.(3.12) for bem condicionada ou, mais especificamente, se for inversível e limitada.

$$
\Phi=\sum_{i=0}^{s-1} \varphi(k-i) \varphi(k-i)^{T}
$$

$\mathrm{Na}$ eq.(3.12), s é a quantidade de dados considerada, também conhecida como janela de identificação.

A condição para que $\Phi$ seja bem condicionada é justamente o critério de excitação persistente forte ("strong persistent excitation - PE - condition") definido em Goodwin e Sin (1984), que exige a existência de dois números positivos $\rho_{0}$ e $\rho_{1}$, tais que:

$$
\rho_{0} \boldsymbol{I} \preceq \sum_{i=0}^{s-1} \varphi(k-i) \varphi(k-i)^{T} \preceq \rho_{1} \boldsymbol{I}
$$


Na equação (3.13), a notação matricial $\boldsymbol{A} \succeq \boldsymbol{B}$ significa que a matriz $\boldsymbol{A}-\boldsymbol{B}$ é positiva semidefinida.

O limite superior da eq.(3.13) é a condição para que a matriz de informação $\Phi$ seja limitada. No caso do MPC onde existem restrições nas entradas, ou seja, onde $\boldsymbol{u}^{\min } \leq \boldsymbol{u}(k+j) \leq \boldsymbol{u}^{\max } \quad(j=0,1, \ldots, m-1)$, a existência de $\rho_{1}$ é garantida devido à própria limitação dos componentes individuais da matriz $\Phi$.

No MPCl de Genceli e Nikolaou (1996), onde os parâmetros do modelo são calculados e atualizados a cada instante de amostragem, a condição de excitação persistente descrita na eq.(3.13) é colocada como uma restrição do problema de otimização com $s=m$, ou seja, com a largura da janela de identificação igual ao horizonte de controle. Neste caso, é direto demonstrar que para que a matriz de informação $\Phi$ não seja singular é necessário que o horizonte de controle seja maior que o número de parâmetros a serem calculados a cada instante de tempo $(s=m \geq n+1)$.

Resumindo o exposto nos dois últimos parágrafos, a restrição de excitação utilizada por Genceli e Nikolaou (1996) é a seguinte:

$$
\sum_{j=0}^{m-1} \boldsymbol{\varphi}(k-j+i) \boldsymbol{\varphi}(k-j+i)^{T} \succeq\left(\rho_{0}-\mu\right) \boldsymbol{I} \succ 0 \quad, \quad i=1,2, \ldots,(m+n-1)
$$

Onde $\mu$ é uma variável de relaxação incluída para garantir que a restrição da eq.(3.14) não seja inviável. O valor ideal desta variável é zero e, portanto, a funçãoobjetivo também é modificada incluindo um termo que força a minimização da variável $\mu$. A necessidade para que a restrição da eq.(3.14) seja definida em todos os instantes de tempo $i=1,2, \ldots,(m+n-1)$ incluindo, portanto, requisitos de excitação persistente (PE) não só das entradas futuras, mas também na relação destas com as entradas passadas, advém também do fato de que os parâmetros do modelo devem ser calculados e atualizados em cada instante de amostragem. 
A variável de relaxação $\mu$ pode ser uma variável escalar, como indicado na eq.(3.14), ou pode ser tratada como um vetor de variáveis $\mu_{i}$ (uma para cada instante $i$ ), como adotado em Eker e Nikolaou (1999).

Shouche et al. (1998) estendem a condição de excitação persistente da eq.(3.14) para o caso SISO onde o modelo considerado está no formato ARX (do inglês "AutoRegressive with eXogenous input") abaixo:

$$
y(k)=\sum_{i=1}^{n a} a_{i} y(k-i)+\sum_{i=1}^{n b} b_{i} u(k-i)+d+\varepsilon(k)
$$

Onde:

$a_{i} \quad$ são os parâmetros do polinômio do denominador da função de transferência discreta do modelo $-A(z)=1+a_{1} z^{-1}+a_{2} z^{-2}+\cdots+a_{n a} z^{-n a}$

$b_{i} \quad$ são os parâmetros do polinômio do numerador da função de transferência discreta do modelo $-B(z)=b_{1} z^{-1}+b_{2} z^{-2}+\cdots+b_{n b} z^{-n b}$

na ordem do denominador da função de transferência discreta do modelo

$n b$ ordem do numerador da função de transferência discreta do modelo

$d$ perturbação constante

$\varepsilon(k)$ erro de predição (ou resíduo) do modelo, no instante $k$

Neste caso, o vetor de regressores $\varphi(k)$ abaixo indicado inclui tanto termos das entradas quanto das saídas.

$$
\varphi(k)=[y(k-1), \ldots, y(k-n a), u(k-1), \ldots, u(k-n b), 1]^{T}
$$

Em conseqüência, a matriz de informação $\Phi$ definida na eq.(3.12) também é função das entradas e saídas simultaneamente.

Para a formulação da restrição de excitação a ser inserida no MPC, eq.(3.14), é conveniente que esta contenha apenas termos nas entradas. No trabalho de Shouche (1996) é demonstrado que se a matriz $\Psi$ na eq.(3.17) for inversível é 
garantido que também a matriz de informação $\Phi$, definida na eq.(3.18) para o caso ARX em consideração, será inversível.

$$
\begin{aligned}
& \Psi=\sum_{i=0}^{p-1} \boldsymbol{\psi}(k-i) \boldsymbol{\psi}(k-i)^{T} \\
& \boldsymbol{\Phi}=\sum_{i=0}^{s-1} \boldsymbol{\varphi}(k-i) \varphi(k-i)^{T}
\end{aligned}
$$

Onde

$$
\begin{aligned}
& \Psi(k)=[u(k-i), \ldots, u(k-i-n a-n b+1), 1]^{T} \\
& \varphi(k)=[y(k-i-1), \ldots, y(k-i-n a), u(k-i-1), \ldots, u(k-i-n b), 1]^{T} \\
& p \leq s-n a .
\end{aligned}
$$

Portanto, também neste caso (ARX), a restrição de excitação definida na eq.(3.14) pode continuar a ser usada, só que agora o horizonte de controle deve ser tal que $m \geq n b+n a+1$ e o vetor $\varphi(k)$ deve ter seu número de termos redimensionado para $(n a+n b+1)$, passando a ser denominado $\psi(k)$. Assim, para não ferir as definições usualmente empregadas em identificação de sistemas, convencionou-se neste trabalho chamar a nova matriz $\Psi$ definida na eq.(3.17) de pseudo-matriz de informação e o vetor $\psi(k)$ de pseudo-vetor de regressores.

A condição básica a ser respeitada para o caso SISO é que o rank da matriz considerada na restrição de excitação deve ser igual ou maior que o número de parâmetros do modelo a ser identificado.

A comprovação desta condição e a extensão para o caso MIMO podem ser vistas em Shouche (1996).

Para o controlador empregado neste trabalho, como o modelo OPOM é incremental nas entradas, também a formulação da restrição de excitação emprega variações nas entradas $\Delta \boldsymbol{u}(k+j), j=0,1, \ldots, m-1$, no lugar das entradas $\boldsymbol{u}(k+j)$ em si. 
Assim, a restrição de excitação efetivamente empregada nas formulações deste capítulo no geral e nos casos simulados no capítulo 4 em particular utiliza a seguinte pseudo-matriz de informação:

$$
\Psi_{i}=\sum_{j=0}^{m-1} \psi(k-j+i / k) \psi(k-j+i / k)^{T} \quad, \quad i=1,2, \ldots, m
$$

Onde

$$
\psi(k-j+i / k)=\left[\Delta \boldsymbol{u}(k-j+i-1 / k)^{T}, \Delta \mathbf{u}(k-j+i-2 / k)^{T}, \ldots, \Delta \boldsymbol{u}(k-j+i-N / k)^{T}\right]^{T}
$$

$N \quad$ é o número de termos do pseudo-vetor de regressores considerado.

Observe-se que o vetor $\psi(k-j+i / k)$ acima difere um pouco do apresentado na eq.(3.17) não só por considerar $\Delta \boldsymbol{u}$ no lugar de $\boldsymbol{u}$, mas também por não conter o termo constante "1". Isto porque no caso do controlador agora considerado, o vetor correspondente à perturbação $\boldsymbol{d}$ é calculado a cada instante de amostragem, conforme a formulação tradicional do MPC, pela diferença entre o valor medido das entradas no instante $k T$ e a predição das entradas no instante $k T$ efetuada no instante anterior $(k-1) T$, ou seja:

$$
\boldsymbol{d}(k)=\boldsymbol{y}_{p}(k)-\boldsymbol{y}(k / k-1)
$$

A utilização da pseudo-matriz de informação definida na eq.(3.19) variará de acordo com a abordagem usada para a formulação da restrição de excitação, conforme descrito nas seções seguintes.

\subsection{CONTROLADOR COM MAXIMIZAÇÃO DA EXCITAÇÃO}

Nesta abordagem, que se convencionou chamar de "Problema (P1)", simplesmente se inseriu a restrição de excitação no MPC convencional apresentado no "Problema (POa)" da seção 2.2.4 com o objetivo de observar os efeitos produzidos no comportamento do controlador. Neste caso, adotando abordagem similar ao $\mathrm{MPCl}$ 
de Shouche et al. (1998), a função-objetivo teve que ser modificada para inserir um termo que procura maximizar a excitação das variáveis de entrada.

Problema (P1)

$$
\begin{aligned}
\min _{\Delta \boldsymbol{u}_{k}, \rho} J_{K}= & \sum_{j=0}^{n p}\left[\boldsymbol{y}(k+j)-\boldsymbol{y}^{s p}\right]^{T} \boldsymbol{Q}\left[\boldsymbol{y}(k+j)-\boldsymbol{y}^{s p}\right]+ \\
& +\sum_{j=0}^{m-1} \Delta \boldsymbol{u}(k+j)^{T} \boldsymbol{R} \Delta \boldsymbol{u}(k+j)-q_{1} \rho^{2}
\end{aligned}
$$

Sujeito a

$$
\begin{aligned}
& -\Delta \boldsymbol{u}^{\max } \leq \Delta \boldsymbol{u}(k+j) \leq \Delta \boldsymbol{u}^{\max } \quad, \quad j=0,1, \ldots, m-1 \\
& \Delta \boldsymbol{u}(k+j)=0, j \geq m \\
& \boldsymbol{u}^{\min } \leq \boldsymbol{u}(k+j) \leq \boldsymbol{u}^{\max }, j=0,1, \ldots, m-1 \\
& \Psi_{i}=\sum_{j=0}^{m-1} \Psi(k-j+i / k) \psi(k-j+i / k)^{T}, i=m \\
& \Psi_{i} \succeq \rho \boldsymbol{I} \quad, \quad i=m \\
& \rho \geq \rho_{0}
\end{aligned}
$$

Onde

$\psi(k-j+i / k)=\left[\Delta \boldsymbol{u}(k-j+i-1 / k)^{T}, \Delta \boldsymbol{u}(k-j+i-2 / k)^{T}, \ldots, \Delta \boldsymbol{u}(k-j+i-N / k)^{T}\right]^{T}$

Neste caso, $\rho$ é uma nova variável introduzida no problema de otimização relacionada à excitação introduzida nas variáveis manipuladas, enquanto $\rho_{0}$ é um parâmetro que define o nível mínimo de excitação desejado. Já $q_{1}$ é o peso da excitação na função-objetivo, um novo parâmetro de sintonia do controlador que penaliza (neste caso, valoriza) a excitação.

Pela eq.(3.21) nota-se que a função-objetivo tentará maximizar a excitação $\rho$, uma vez que o termo correspondente a esta variável aparece com sinal negativo. Observe-se que a maximização desta variável de excitação, desejável para os fins 
de identificação a que se destina, é conflitante com as demais parcelas da funçãoobjetivo correspondentes aos erros entre as saídas medidas e os set-points e às variações nas manipuladas (entradas), pois a maximização da variável de excitação implica diretamente na maior variação das manipuladas e, conseqüentemente, em maior erro nas controladas. Desta forma, a quantidade de excitação produzida será função da sintonia adotada, ou seja, dos valores atribuídos aos parâmetros $\boldsymbol{Q}, \boldsymbol{R}$ e $q_{1}$.

Nas eq.(3.25) e (3.26) a restrição de excitação só foi estabelecida para um único instante de tempo $i=m$, diferente do adotado nos trabalhos de Genceli e Nikolaou (1996) e Shouche (1996). Isto pode ser justificado da seguinte forma: nos estudos citados se emprega a identificação e atualização do modelo "on-line" e, portanto, busca-se garantir que em cada instante de tempo dentro do horizonte de controle, a entrada computada seja persistentemente excitante em relação às entradas passadas (ou futuras anteriores ao instante considerado). Para garantir a inversibilidade da matriz de informação e o cálculo "on-line" dos parâmetros do modelo a ser atualizado, se adota uma janela de identificação móvel e pequena, da mesma ordem de grandeza do horizonte de controle, sendo fundamental se manter uma seqüência de entradas onde a condição de persistência de excitação (PE) seja também garantida em todos os instantes de tempo. Para que tal problema de otimização seja viável, é empregada uma rotina de inicialização que faz com que as entradas passadas tenham sido computadas respeitando a mesma condição PE imposta para as entradas futuras.

Já no presente "Problema (P1)", embora a intenção também seja fazer com que o sistema seja excitado de forma consistente, optou-se por não complicar desnecessariamente o problema de otimização e se introduziu uma restrição de excitação num único instante de tempo que contemple apenas as variações futuras das entradas dentro do horizonte de controle $(\Delta \boldsymbol{u}(k), k=0,1, \ldots, m)$, de forma que estas respeitem a condição de excitação persistente (PE). Isto porque neste trabalho se pretende realizar a re-identificação do modelo de forma "off-line", com uma grande quantidade de dados (não se empregou o conceito de janela de identificação móvel e identificação "on-line" do MPCl anteriormente descrito). Ou seja, no atual 
problema de otimização procura-se não se preocupar com as entradas passadas, já implementadas, uma vez que não se tem ação sobre as mesmas.

A eq.(3.21) pode ser reescrita para a formulação OPOM apresentada em 2.2.4 e usada nos nossos testes, conforme abaixo:

$$
\min _{\Delta \boldsymbol{u}_{k}, \rho} J_{K}=\Delta \boldsymbol{u}_{k}{ }^{T} \boldsymbol{H} \Delta \boldsymbol{u}_{k}+2 \boldsymbol{c}_{\boldsymbol{f}}{ }^{T} \Delta \boldsymbol{u}_{k}+c-q_{1} \rho^{2}
$$

Esta formulação do "Problema (P1)" foi utilizada apenas para se verificar se era possível a observação prática da excitação das variáveis de entrada. As figuras $3.1 \mathrm{e}$ 3.2 ilustram as oscilações provocadas na malha de controle de uma coluna debutanizadora, descrita adiante com maiores detalhes na seção 4.1, para três diferentes ajustes dos parâmetros de sintonia apresentados na tabela 3.1.

Tabela 3.1 - Sintonia empregada nos testes do controlador do Problema (P1)

\begin{tabular}{|c|c|c|c|c|}
\hline \multicolumn{5}{|c|}{ Parâmetros fixos } \\
\hline Horizonte de predição $(n p)$ & 40 & \multirow{3}{*}{$\begin{array}{l}\text { Limites nas } \\
\text { manipuladas }\end{array}$} & $u^{\max }$ & {$\left[\begin{array}{ll}5 & 5\end{array}\right]$} \\
\hline Horizonte de controle $(m)$ & 7 & & $u^{\min }$ & {$\left[\begin{array}{ll}-10 & -10\end{array}\right]$} \\
\hline Set-point controladas $\left(y^{s p}\right)$ & {$\left[\begin{array}{ll}0 & 0\end{array}\right]$} & & $\Delta \boldsymbol{u}^{\max }$ & {$\left[\begin{array}{ll}1 & 1\end{array}\right]$} \\
\hline Peso da excitação $(q 1)$ & 100 & \multicolumn{2}{|c|}{ Fator de excitação $\left(\rho_{0}\right)$} & 0.01 \\
\hline \multicolumn{5}{|c|}{ Parâmetros variáveis } \\
\hline \multicolumn{2}{|l|}{ Parâmetro } & Caso 1 & Caso 2 & Caso 3 \\
\hline Peso das controladas $(\boldsymbol{Q})$ & & [ 1 1 1 $]$ & {$\left[\begin{array}{ll}10 & 10\end{array}\right]$} & {$\left[\begin{array}{ll}10 & 10\end{array}\right]$} \\
\hline Fator de supressão $(\boldsymbol{R})$ & & {$\left[\begin{array}{ll}0.1 & 0.1\end{array}\right]$} & {$\left[\begin{array}{ll}0.1 & 0.1\end{array}\right]$} & {$\left[\begin{array}{ll}5 & 5\end{array}\right]$} \\
\hline
\end{tabular}

Observa-se nas figuras 3.1 e 3.2 que a introdução da restrição de excitação faz com que as variáveis controladas oscilem em torno dos respectivos "set-points" enquanto as variáveis manipuladas oscilam em torno dos seus valores em regime permanente.

Nesta abordagem - Problema $(P 1)$ - a excitação produzida é função da sintonia empregada, ou seja, dos valores atribuídos aos parâmetros $\boldsymbol{Q}, \boldsymbol{R}$ e $q_{1}$. Pode-se observar que do caso 1 para o caso 2 , um aumento no peso das controladas implicou na redução do nível de excitação produzido. Do caso 2 para o caso 3, um aumento no peso das manipuladas (fatores de supressão) também implicou em redução da excitação. 

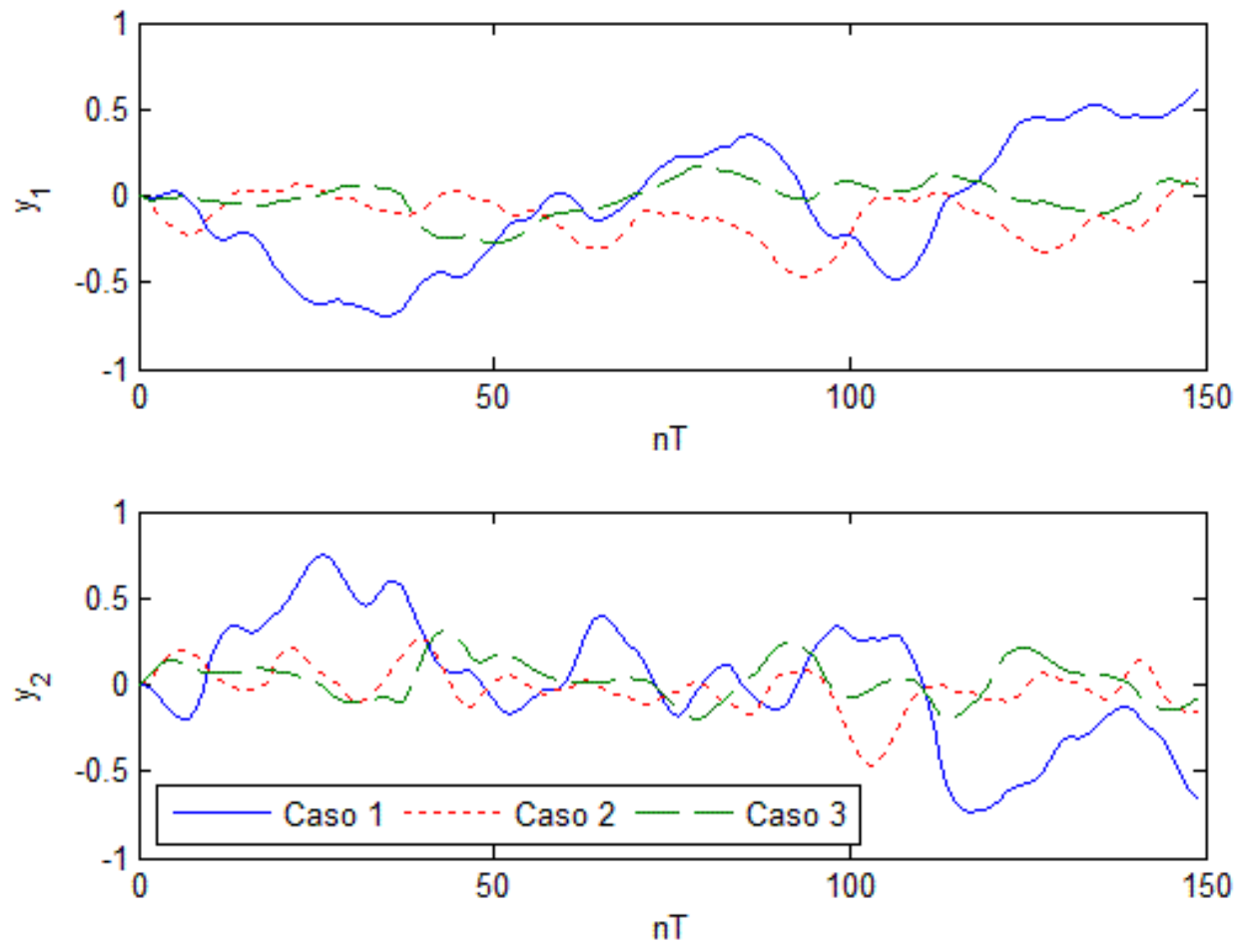

Figura 3.1 - Comportamento das variáveis controladas do controlador com restrição de excitação - Formulação $(P 1)$
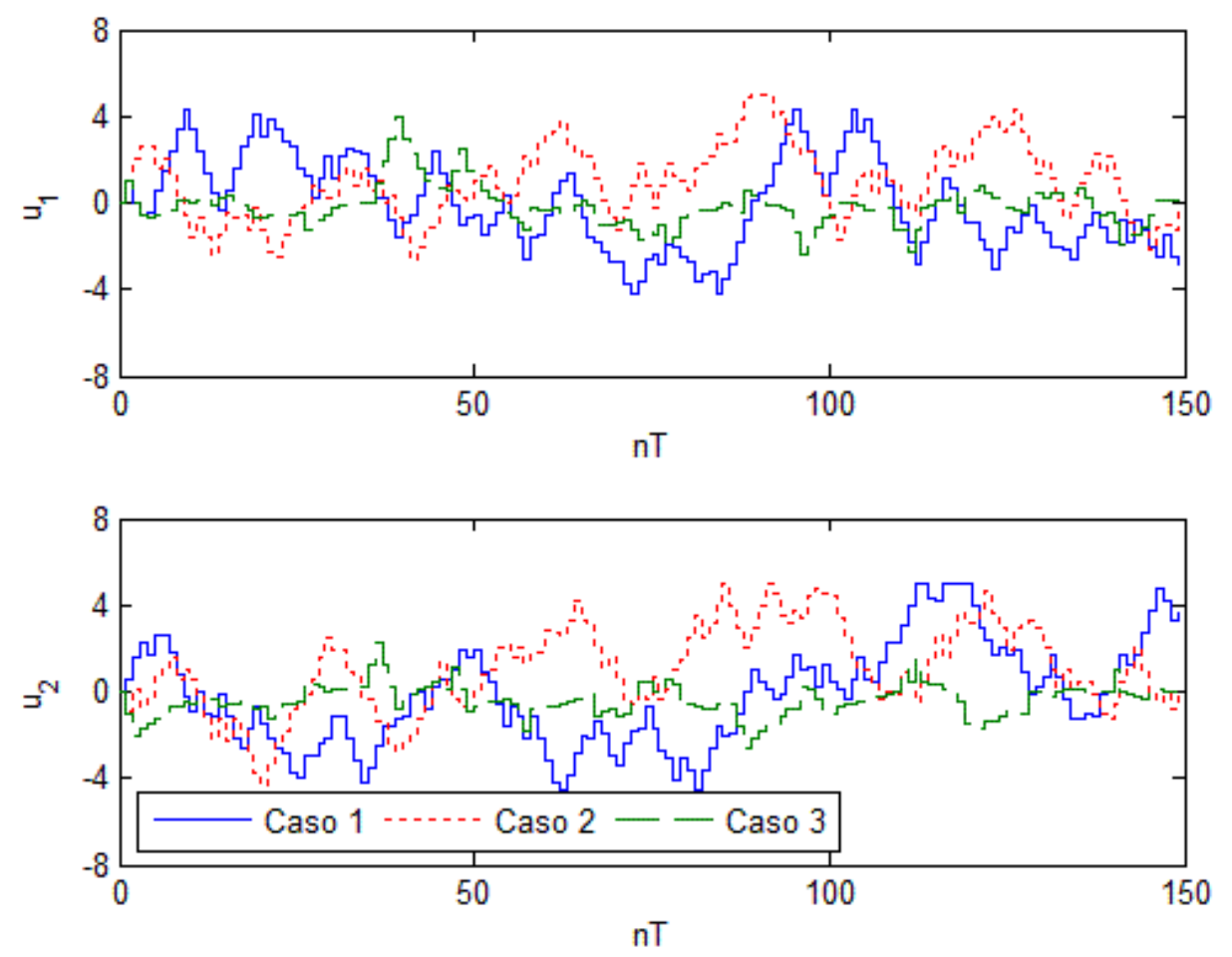

Figura 3.2 - Comportamento das variáveis manipuladas do controlador com restrição de excitação - Formulação (P1) 
Nos exemplos de aplicações descritos adiante no capítulo 4, optou-se por usar as formulações dos "Problemas (P3) e (P4)" apresentadas adiante, por permitirem melhor controle da excitação produzida.

\subsection{CONTROLADOR COM EXCITAÇÃO MÍNIMA E VARIÁVEIS DE FOLGA}

Nesta seção é descrita a implementação no controlador MPC de uma restrição de excitação similar à emprega nos estudos de Genceli e Nikolaou (1996) e Shouche et al. (1998). A diferença em relação ao Problema (P1) é que em vez de se incluir uma variável diretamente relacionada à excitação, agora é incluída uma variável de folga que o problema de otimização procura minimizar.

O novo problema fica, então, assim definido:

Problema (P2)

$$
\begin{aligned}
\min _{\Delta \mathbf{u}_{k}, \mu} J_{K}= & \sum_{j=0}^{n p}\left[\boldsymbol{y}(k+j)-\boldsymbol{y}^{s p}\right]^{T} \boldsymbol{Q}\left[\boldsymbol{y}(k+j)-\boldsymbol{y}^{s p}\right]+ \\
& +\sum_{j=0}^{m-1} \Delta \boldsymbol{u}(k+j)^{T} \boldsymbol{R} \Delta \boldsymbol{u}(k+j)+q_{2} \mu^{2}
\end{aligned}
$$

Sujeito a

$$
\begin{aligned}
& -\Delta \boldsymbol{u}^{\max } \leq \Delta \boldsymbol{u}(k+j) \leq \Delta \boldsymbol{u}^{\max } \quad, \quad j=0,1, \ldots, m-1 \\
& \Delta \boldsymbol{u}(k+j)=0, j \geq m \\
& \boldsymbol{u}^{\min } \leq \boldsymbol{u}(k+j) \leq \boldsymbol{u}^{\max }, j=0,1, \ldots, m-1 \\
& \Psi_{i}=\sum_{j=0}^{m-1} \psi(k-j+i / k) \psi(k-j+i / k)^{T}, i=m \\
& \Psi_{i} \succeq\left(\rho_{0}-\mu\right) \boldsymbol{I} \quad, \quad i=m \\
& \rho_{0}-\mu \geq 0
\end{aligned}
$$


Onde

$\psi(k-j+i / k)=\left[\Delta \boldsymbol{u}(k-j+i-1 / k)^{T}, \Delta \boldsymbol{u}(k-j+i-2 / k)^{T}, \ldots, \Delta \boldsymbol{u}(k-j+i-N / k)^{T}\right]^{T}$

$q_{2}$ é o peso da variável de folga $\mu$

Nesta formulação, $\mu$ é uma variável de folga incluída para garantir a viabilidade da restrição indicada na eq.(3.34) e que tende a ser minimizada pelo problema de otimização. O valor ideal para esta variável é zero, uma vez que ela relaxa a condição de excitação.

Da mesma forma que na abordagem anterior, $\rho_{0}$ é um parâmetro que define o nível mínimo de excitação desejado. É trivial demonstrar que se $\rho_{0}=0$ nenhuma excitação é gerada e o controlador se comporta como um MPC normal.

As figuras 3.3 e 3.4 ilustram exemplos simulados com esta formulação do controlador para diferentes valores do parâmetro $\rho_{0}$. A planta considerada é a mesma coluna debutanizadora usada na seção anterior e a sintonia empregada para o controlador está indicada na tabela 3.2.

Tabela 3.2 - Sintonia empregada nos testes do controlador do Problema (P2)

\begin{tabular}{|c|c|c|c|c|}
\hline \multicolumn{5}{|c|}{ Parâmetros fixos } \\
\hline Horizonte de predição $(n p)$ & 40 & \multirow{3}{*}{$\begin{array}{l}\text { Limites nas } \\
\text { manipuladas }\end{array}$} & $u^{\max }$ & {$\left[\begin{array}{ll}15 & 15\end{array}\right]$} \\
\hline Horizonte de controle $(m)$ & 7 & & $u^{\min }$ & {$\left[\begin{array}{ll}-10 & -10\end{array}\right]$} \\
\hline Set-point controladas $\left(y^{s p}\right)$ & {$\left[\begin{array}{ll}0 & 0\end{array}\right]$} & & $\Delta \boldsymbol{u}^{\max }$ & {$\left[\begin{array}{ll}1 & 1\end{array}\right]$} \\
\hline Peso das controladas $(\boldsymbol{Q})$ & {$\left[\begin{array}{ll}10 & 10\end{array}\right]$} & \multicolumn{2}{|c|}{ Fator de supressão $(\boldsymbol{R})$} & {$\left[\begin{array}{ll}5 & 5\end{array}\right]$} \\
\hline Peso da folga $(q 2)$ & 50 & & & \\
\hline \multicolumn{5}{|c|}{ Parâmetro variável } \\
\hline \multicolumn{2}{|l|}{ Parâmetro } & Caso 1 & Caso 2 & Caso 3 \\
\hline \multicolumn{2}{|l|}{ Fator de excitação $\left(\rho_{0}\right)$} & 2 & 0.5 & 0 \\
\hline
\end{tabular}



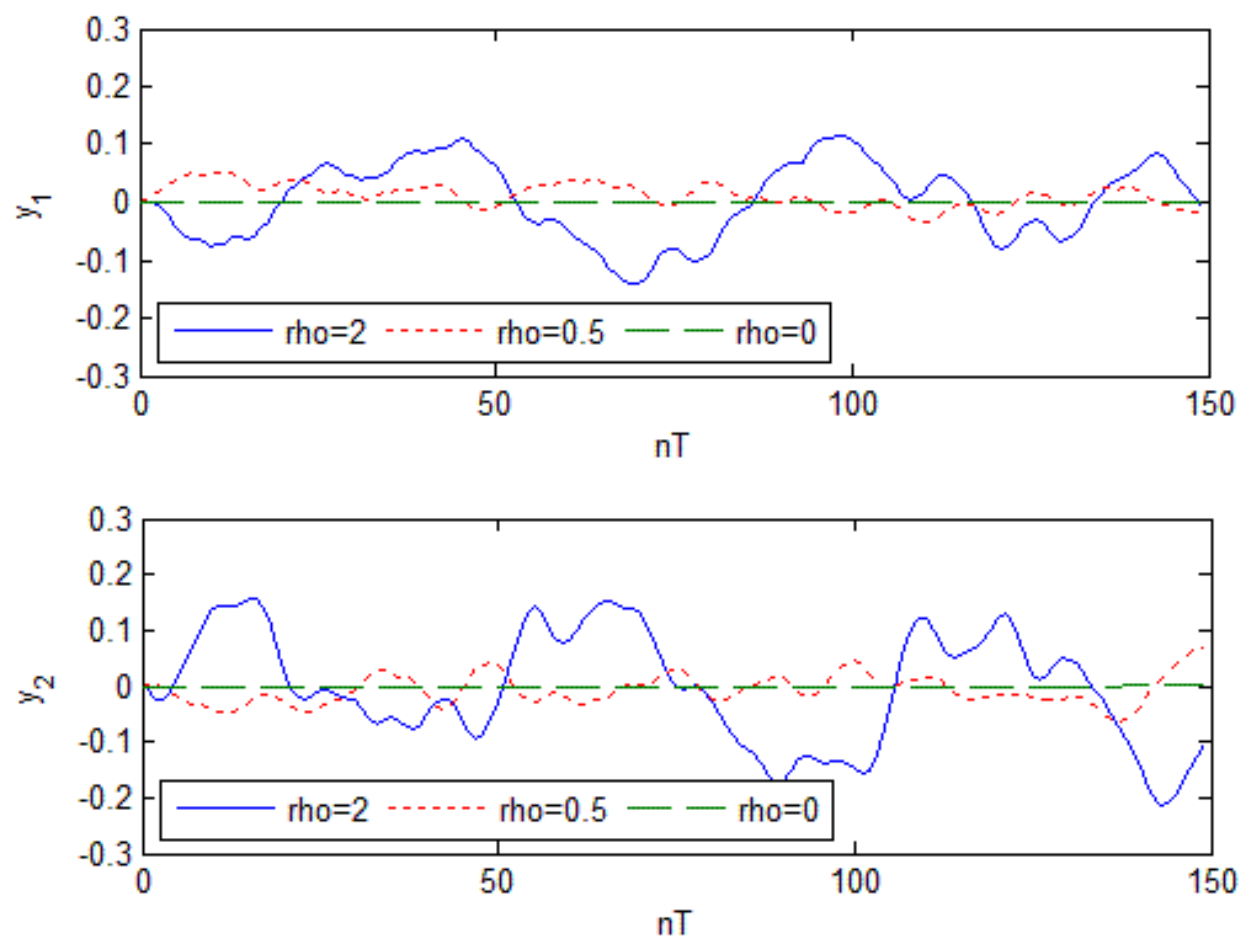

Figura 3.3 - Comportamento das variáveis controladas do controlador com restrição de excitação - Formulação (P2)
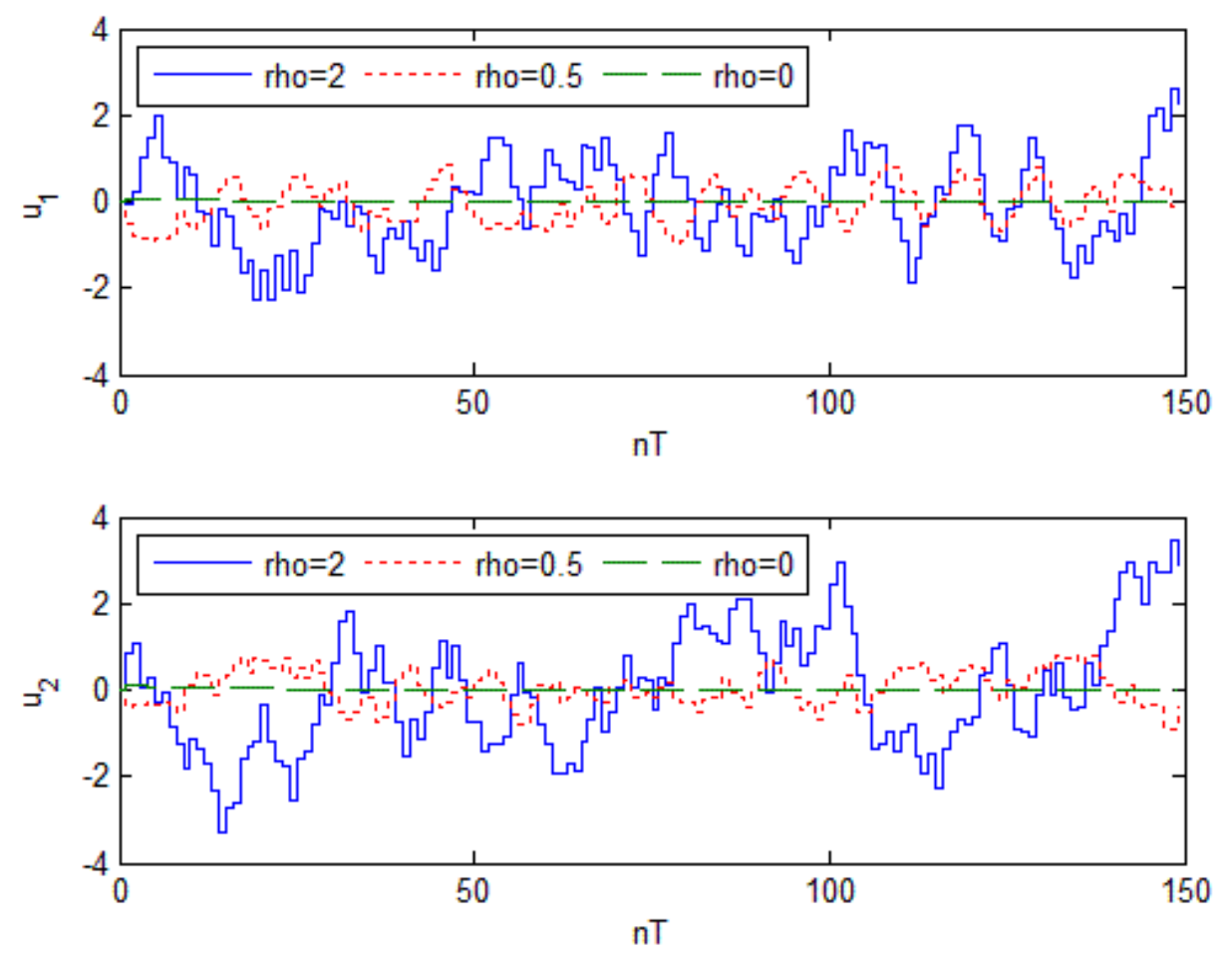

Figura 3.4 - Comportamento das variáveis manipuladas do controlador com restrição de excitação - Formulação (P2) 
Como se pode observar, as variações produzidas nas variáveis controladas e manipuladas em torno dos "set-points" e dos valores em regime permanente, respectivamente, foram diretamente proporcionais ao valor definido para o nível mínimo de excitação desejado (fator $\rho_{0}$ ). Observa-se ainda que a definição de excitação mínima nula automaticamente indica que não se deseja utilizar a restrição de excitação para fins de identificação, mas sim o controlador MPC convencional. Este comportamento representa uma vantagem em relação à formulação anterior, uma vez que naquela a definição de uma excitação mínima igual a zero não implica no "desligamento" da função de excitação do controlador, pois o controlador do Problema ( $P 1)$ sempre procura maximizar a excitação.

\subsection{CONTROLADOR COM RESTRIÇÃO NO VALOR DA FUNÇÃO-OBJETIVO}

Nesta formulação, a cada instante de tempo, dois problemas de otimização são resolvidos:

a) Primeiramente, é resolvido o problema do MPC convencional, sem excitação, o qual fornece os valores ótimos das variações nas entradas $\Delta u^{*}(k)$ e 0 correspondente valor mínimo da função-objetivo $J_{K 0}$ (entenda-se por 'valores ótimos' os valores das variações nas variáveis manipuladas produzidos por um algoritmo MPC convencional).

b) Na seqüência, é resolvido o problema de otimização com restrição de excitação cuja função-objetivo é simplesmente maximizar a excitação, estabelecendo-se um limite (folga) para o valor da função-custo equivalente do MPC com excitação $\left(J_{K}\right)$ em relação ao valor $J_{K 0}$ computado na etapa a.

O primeiro subproblema (etapa a) já foi formulado na seção 2.2.4, Problema (POa). Já o subproblema da etapa b fica assim formulado: 
Problema (P3)

$\max _{\Delta \boldsymbol{u}_{k}, \rho} \rho$

Sujeito a

$$
\begin{aligned}
& -\Delta \boldsymbol{u}^{\max } \leq \Delta \boldsymbol{u}(k+j) \leq \Delta \boldsymbol{u}^{\max } \quad, \quad j=0,1, \ldots, m-1 \\
& \Delta \boldsymbol{u}(k+j)=0 \quad, \quad j \geq m \\
& \boldsymbol{u}^{\min } \leq \boldsymbol{u}(k+j) \leq \boldsymbol{u}^{\max }, j=0,1, \ldots, m-1 \\
& \Psi_{i} \succeq \rho \boldsymbol{I} \quad, \quad i=m \\
& \rho \geq \rho_{0} \\
& J_{K} \leq K_{e 1} J_{K 0}+K_{e 2}
\end{aligned}
$$

Onde

$$
\begin{aligned}
& \Psi_{i}=\sum_{j=0}^{m-1} \boldsymbol{\psi}(k-j+i / k) \boldsymbol{\psi}(k-j+i / k)^{T} \quad, \quad i=m \\
& \boldsymbol{\Psi}(k-j+i / k)=\left[\Delta \boldsymbol{u}(k-j+i-1 / k)^{T}, \Delta \boldsymbol{u}(k-j+i-2 / k)^{T}, \ldots, \Delta \boldsymbol{u}(k-j+i-N / k)^{T}\right]^{T} \\
& J_{K}=\sum_{j=0}^{n p}\left[\boldsymbol{y}(k+j)-\boldsymbol{y}^{s p}\right]^{T} \boldsymbol{Q}\left[\boldsymbol{y}(k+j)-\boldsymbol{y}^{s p}\right]+\sum_{j=0}^{m-1} \Delta \boldsymbol{u}(k+j)^{T} \boldsymbol{R} \Delta \boldsymbol{u}(k+j)
\end{aligned}
$$

$J_{K 0}$ é o valor da função-objetivo do MPC convencional - Problema (P0.a), calculado no mesmo instante de tempo.

$K_{e 1}, K_{e 2}$ são parâmetros que definem a folga em relação ao valor da função-custo do MPC normal. Indiretamente, definem a quantidade de excitação ou perturbação admitida no sistema.

Assim, pode-se definir o algoritmo abaixo para este controlador, a ser empregado a cada instante de tempo. 
Algoritmo 3.1

1) Resolver o problema de otimização correspondente ao MPC convencional, Problema (POa), obtendo os valores das variações nas entradas, $\Delta \boldsymbol{u}^{*}(k)$, e o valor da correspondente função-objetivo $J_{K 0}$, a ser passado como parâmetro para o problema do passo 2.

a) Armazenar os valores das variações nas entradas $-\Delta \boldsymbol{u}^{*}(k)$ - calculados no passo 1.

2) Resolver o problema de otimização do controlador com restrição de excitação, Problema (P3), usando como estimativa inicial os valores armazenados na etapa 1.a.

3) Atualizar a saída e o estado do modelo. Aplicar a primeira ação de controle à planta, utilizando o seguinte critério:

a) Se o problema da etapa 2 for viável, usar as variações nas entradas calculadas nesta etapa.

b) Se o problema da etapa 2 for inviável, usar as variações nas entradas armazenadas na etapa 1.a, $\Delta \boldsymbol{u}^{*}(k)$.

4) Passar para o próximo instante de tempo, retornando à etapa 1.

Com este algoritmo o controlador proporcionará a máxima excitação possível a cada instante de tempo, sendo esta limitada pelas restrições normais do MPC, pela sintonia empregada e pela restrição da eq.(3.42).

Outra característica desta abordagem é que quando o subproblema com restrição de excitação for inviável, o controlador se comportará como um MPC convencional, retomando a função de excitação assim que as condições de entrada e saída do sistema propiciem a viabilidade do (sub) Problema (P3). 
As figuras 3.5 e 3.6 ilustram exemplos do comportamento desta formulação do controlador para a mesma coluna debutanizadora usada nos exemplos das seções anteriores, para três ajustes distintos definidos na tabela 3.3.

Tabela 3.3 - Sintonia empregada nos testes do controlador do Problema (P3)

\begin{tabular}{|c|c|c|c|c|}
\hline \multicolumn{5}{|c|}{ Parâmetros fixos } \\
\hline Horizonte de predição $(n p)$ & 20 & \multirow{3}{*}{$\begin{array}{l}\text { Limites nas } \\
\text { manipuladas }\end{array}$} & $\boldsymbol{u}^{\max }$ & {$\left[\begin{array}{ll}15 & 15\end{array}\right]$} \\
\hline Horizonte de controle $(m)$ & 7 & & $u^{\min }$ & {$\left[\begin{array}{ll}-10 & -10\end{array}\right]$} \\
\hline Set-point controladas $\left(\boldsymbol{y}^{s p}\right)$ & {$\left[\begin{array}{ll}0 & 0\end{array}\right]$} & & $\Delta \boldsymbol{u}^{\max }$ & {$\left[\begin{array}{ll}1 & 1\end{array}\right]$} \\
\hline Peso das controladas $(\boldsymbol{Q})$ & {$\left[\begin{array}{ll}1 & 1\end{array}\right]$} & \multicolumn{2}{|c|}{ Fator de supressão $(\boldsymbol{R})$} & {$\left[\begin{array}{ll}0.1 & 0.1\end{array}\right]$} \\
\hline \multicolumn{5}{|c|}{ Parâmetros variáveis } \\
\hline \multicolumn{2}{|l|}{ Parâmetro } & Caso 1 & Caso 2 & Caso 3 \\
\hline \multicolumn{2}{|l|}{ Fator de folga de excitação $1\left(K_{e 1}\right)$} & 1.5 & 0.5 & 0.2 \\
\hline \multicolumn{2}{|l|}{ Fator de folga de excitação $2\left(K_{e 2}\right)$} & 2 & 1 & 0.5 \\
\hline
\end{tabular}
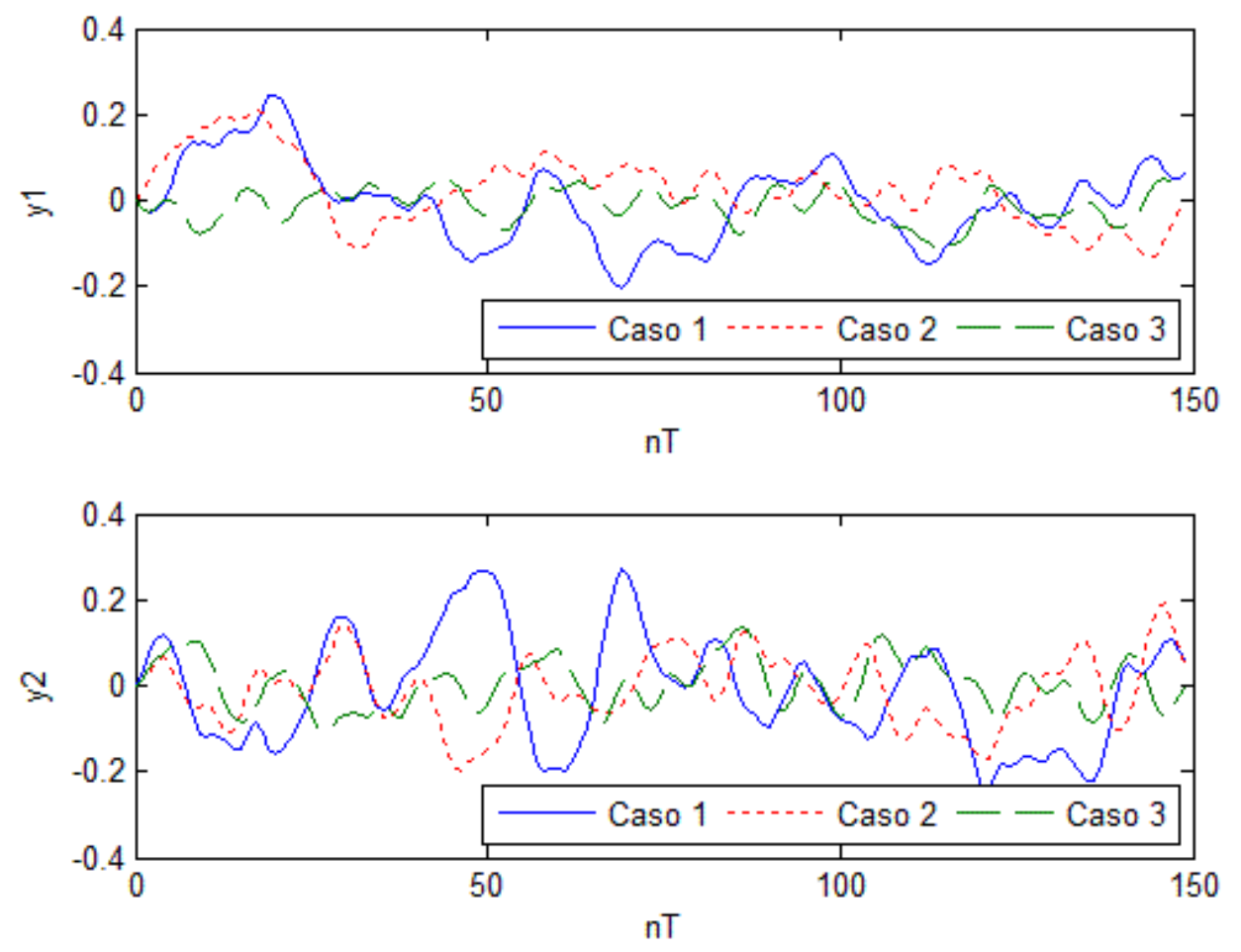

Figura 3.5 - Comportamento das variáveis controladas do controlador com restrição de excitação - Formulação (P3) 

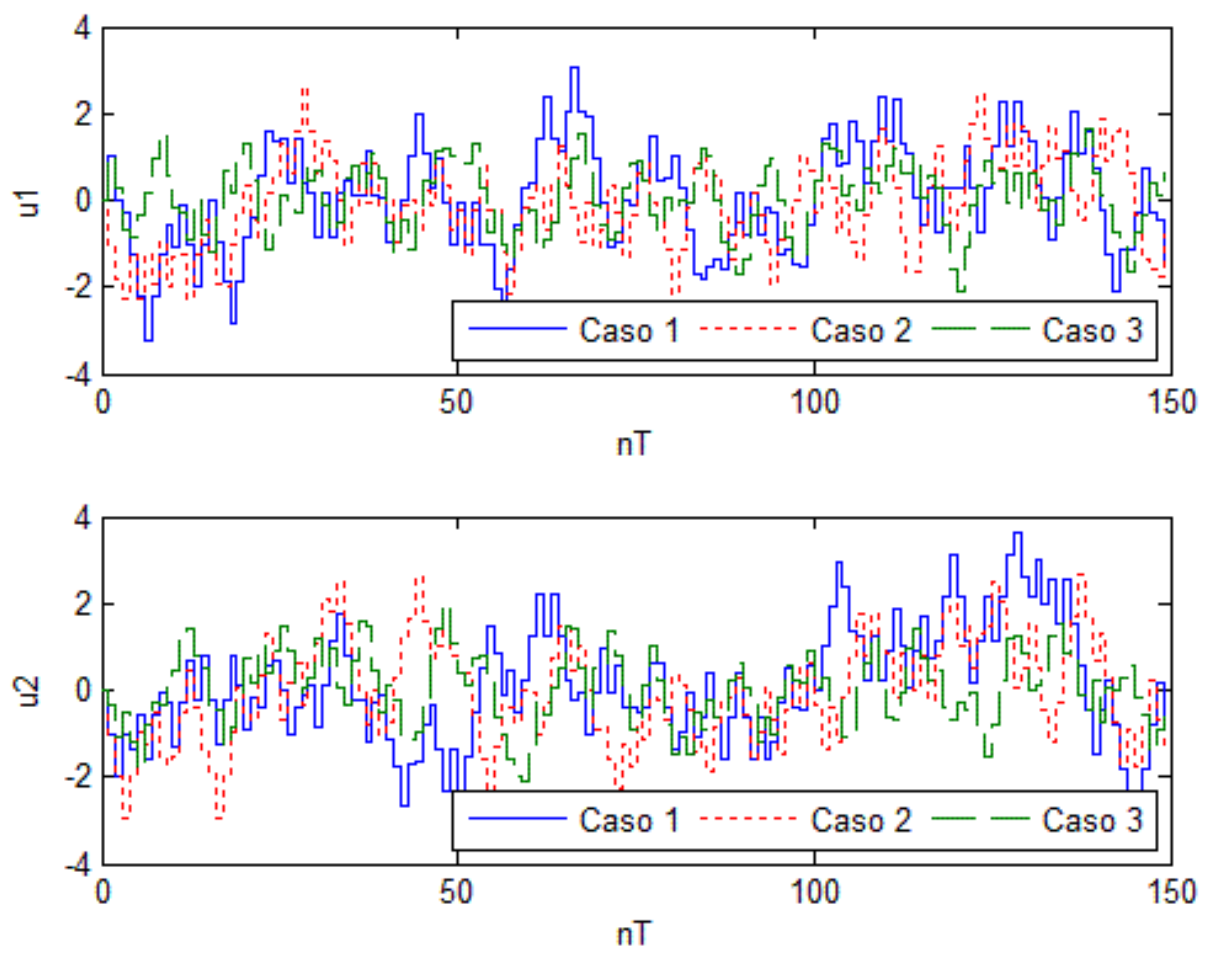

Figura 3.6 - Comportamento das variáveis manipuladas do controlador com restrição de excitação - Formulação (P3)

Como se pode observar, a escolha adequada dos fatores de folga permite o controle da excitação provocada nas variáveis manipuladas e o conseqüente controle da variação nas variáveis controladas em torno dos respectivos "set-points".

Além da observação gráfica do comportamento das variáveis, uma avaliação quantitativa do nível de excitação introduzido na malha de controle pode ser obtida pela comparação da variância dos sinais de entrada e saída (variáveis manipuladas e controladas) para os três casos. A tabela 3.4 resume os valores das variâncias observadas nos três exemplos ilustrados nas figuras 3.5 e 3.6.

Tabela 3.4 - Variância dos sinais de entrada e saída para os exemplos das figuras 3.5 e 3.6

\begin{tabular}{|c|c|c|c|c|c|c|}
\cline { 4 - 7 } \multicolumn{2}{c|}{} & \multicolumn{4}{c|}{ Variâncias } \\
\hline Caso & $K_{e 1}$ & $K_{e 2}$ & $y_{1}$ & $y_{2}$ & $u_{1}$ & $u_{2}$ \\
\hline Caso 1 & 1.5 & 2 & 0.0089 & 0.0163 & 1.5007 & 1.7268 \\
\hline Caso 2 & 0.5 & 1 & 0.0062 & 0.0068 & 1.1320 & 1.4192 \\
\hline Caso 3 & 0.2 & 0.5 & 0.0015 & 0.0033 & 0.6120 & 0.7050 \\
\hline
\end{tabular}


Pode-se constatar que a redução nos valores dos fatores de folga da função-objetivo do subproblema com excitação em relação ao subproblema sem excitação provoca a correspondente redução nas variâncias dos sinais de entrada e saída. Observa-se também que esta relação não é linear.

\subsection{CONTROLADOR POR FAIXAS COM RESTRIÇÃO NO VALOR DA FUNÇÃO- OBJETIVO}

De forma similar ao empregado na seção 3.5, nesta formulação também são resolvidos dois problemas de otimização a cada instante de tempo:

a) Inicialmente, é resolvido o Problema (POb) do MPC por faixas apresentado na seção 2.3, o qual fornece os valores ótimos das variações nas entradas $\Delta \boldsymbol{u}^{*}(k) \mathrm{e}$ do "set-point" calculado no instante considerado $\boldsymbol{y}^{s p^{*}}(k)$, além do valor da função-objetivo $J_{K 0}$ no instante considerado para o controlador por faixas e sem excitação.

b) Em seguida, é resolvido o problema de otimização com restrição de excitação. Neste problema, a função-objetivo é a maximização da excitação, onde se define um limite (folga) para o valor da função-custo do MPC com excitação $\left(J_{K}\right)$ em relação ao valor $J_{K 0}$ computado na etapa a.

A formulação do primeiro subproblema (etapa a) já foi definida na seção 2.3, Problema (POb), enquanto o subproblema da etapa b pode ser assim formulado:

Problema (P4)

$\max _{\Delta \boldsymbol{u}_{k}, \rho} \rho$

Sujeito a

$$
-\Delta \boldsymbol{u}^{\max } \leq \Delta \boldsymbol{u}(k+j) \leq \Delta \boldsymbol{u}^{\max } \quad, \quad j=0,1, \ldots, m-1
$$




$$
\begin{aligned}
& \Delta \boldsymbol{u}(k+j)=0 \quad, \quad j \geq m \\
& \boldsymbol{u}^{\min } \leq \boldsymbol{u}(k+j) \leq \boldsymbol{u}^{\max } \quad, \quad j=0,1, \ldots, m-1 \\
& \Psi_{i} \succeq \rho \boldsymbol{I} \quad, \quad i=m \\
& \rho \geq \rho_{0} \\
& J_{K} \leq J_{K 0}+K_{e}(k)
\end{aligned}
$$

Onde

$$
\begin{aligned}
& \Psi_{i}=\sum_{j=0}^{m-1} \boldsymbol{\psi}(k-j+i / k) \boldsymbol{\psi}(k-j+i / k)^{T} \quad, \quad i=m \\
& \boldsymbol{\Psi}(k-j+i / k)=\left[\Delta \boldsymbol{u}(k-j+i-1 / k)^{T}, \Delta \boldsymbol{u}(k-j+i-2 / k)^{T}, \ldots, \Delta \boldsymbol{u}(k-j+i-N / k)^{T}\right]^{T} \\
& J_{K}=\sum_{j=1}^{n p} \boldsymbol{e}(k+j)^{T} \boldsymbol{Q} \boldsymbol{e}(k+j)+\sum_{j=0}^{m-1} \Delta \boldsymbol{u}(k+j)^{T} \boldsymbol{R} \Delta \boldsymbol{u}(k+j) \\
& \boldsymbol{e}(k+j)=\boldsymbol{y}(k+j)-\boldsymbol{y}^{s p^{*}}(k) \quad, \quad j=1,2, \ldots, n p
\end{aligned}
$$

$J_{K 0} \quad$ é o valor da função-objetivo do MPC por faixas - Problema (POb), calculado no mesmo instante de tempo.

$K_{e}(k)$ é o fator de folga de excitação, um parâmetro variável calculado a cada instante de tempo que define a folga em relação ao valor da função-objetivo do MPC sem excitação. Define, portanto, a quantidade de excitação ou perturbação admitida no sistema.

Nesta abordagem - Problema (P4) - adotou-se uma estratégia de cálculo do fator de excitação a cada instante de tempo em função do valor da função-objetivo sem excitação no mesmo instante $\left(J_{K 0}\right)$, conforme definido na eq.(3.54) e na figura 3.7, abaixo. 


$$
\begin{aligned}
& K_{e}(k)=J_{K \max }-\frac{J_{K \max }}{J_{K \lim }} \cdot J_{K 0} \quad, 0 \leq J_{K 0}<J_{K \lim } \\
& K_{e}(k)=0, \quad J_{K \lim } \leq J_{K 0}
\end{aligned}
$$

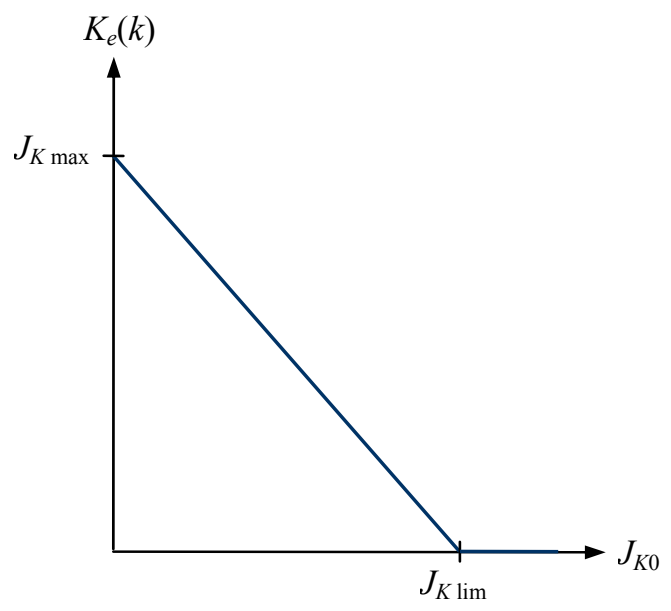

Figura 3.7 - Cálculo do fator de folga de excitação - $K_{e}(k)$

$J_{K \max }$ e $J_{K \lim }$ são parâmetros do controlador que definem o nível de excitação introduzido na malha de controle.

$J_{K \max }$ é calculado através da eq.(3.52) considerando $\boldsymbol{e}(k+j)=0, j=1, \ldots, n p$, e $\Delta \boldsymbol{u}(k+j)=\Delta \boldsymbol{u}^{\max }, \quad j=1, \ldots, m$, o que corresponde ao caso onde todas as variáveis controladas estão dentro das respectivas zonas durante todos os instantes de tempo dentro do horizonte de predição. Nesta condição, a excitação permitida será máxima, o que é obtido com variações nas entradas iguais a $\Delta \boldsymbol{u}^{\max }$ durante todos os instantes dentro do horizonte de controle.

$J_{K \lim }$ também é calculado através da eq.(3.52), porém considerando $\Delta \boldsymbol{u}(k+j)=0, \quad j=1, \ldots, m$, e $\boldsymbol{e}(k+j)=\boldsymbol{e}_{\lim }, j=1, \ldots, n p$, onde o erro limite $\left(\boldsymbol{e}_{\lim }\right)$ pode ser definido em valores absolutos ou como uma fração da faixa de controle definida para as variáveis controladas (p.ex.: $\left.\boldsymbol{e}_{\lim }=\left(\boldsymbol{y}^{\max }-\boldsymbol{y}^{\min }\right) / 2\right)$

A estratégia de cálculo de um fator de folga variável adotada nesta abordagem se mostrou necessária porque o MPC com faixas possui a característica de apresentar 
valores muito pequenos da função-objetivo quando as variáveis controladas se encontram dentro da faixa de controle definida. Isto exigiria valores muito grandes para a folga do subproblema com excitação que, se constantes, poderiam levar à instabilidade ou excitação excessiva quando as variáveis controladas estivessem fora da faixa. Com a estratégia proposta, o fator de folga será maior quando as variáveis controladas estiverem dentro da faixa ( $J_{K 0}$ pequeno) e menor quando estiverem fora da faixa ( $J_{K 0}$ grande). A folga é zerada, resultando em excitação nula, sempre que o valor da função-objetivo sem excitação estiver acima do valor limite definido $\left(J_{K 0}>J_{K \lim }\right)$

Pode-se então definir o algoritmo 3.2 para este controlador, a ser executado a cada instante de tempo.

\section{Algoritmo 3.2}

1) Resolver o problema de otimização correspondente ao MPC por faixas, Problema $(P O b)$, obtendo os valores das variações nas entradas - $\Delta \boldsymbol{u}^{*}(k)$ - para 0 controlador sem excitação, além do valor da correspondente função-objetivo $J_{K 0} \mathrm{e}$ dos "set-points" ótimos $-\boldsymbol{y}^{s p^{*}}(k)$, a serem passados como parâmetros para o problema do passo 3.

a) Armazenar os valores das variações nas entradas calculados no passo 1 , $\Delta \boldsymbol{u}^{*}(k)$.

2) Calcular o valor do fator de excitação $(K e(k))$ em função do valor da funçãoobjetivo sem excitação obtido no passo 1.

3) Resolver o problema de otimização do MPC por faixas com restrição de excitação, Problema (P4), usando como estimativa inicial os valores $\Delta \boldsymbol{u}^{*}(k)$ armazenados na etapa 1.a. 
4) Atualizar a saída e o estado do modelo. Aplicar a primeira ação de controle à planta usando o seguinte critério:

a) Se o problema da etapa 3 for viável, usar as variações nas entradas calculadas nesta etapa.

b) Se o problema da etapa 3 for inviável, usar as variações nas entradas armazenadas na etapa 1.a, $\Delta \boldsymbol{u}^{*}(k)$.

5) Passar para o próximo instante de tempo, retornando à etapa 1.

As figuras 3.8 e 3.9 ilustram exemplos do comportamento desta formulação do controlador para a coluna debutanizadora usada nas seções anteriores. São comparados dois casos, correspondentes a duas sintonias diferentes definidas na tabela 3.5.

Percebe-se nestas figuras que este controlador - Problema (P4) - provoca oscilações nas variáveis manipuladas e controladas enquanto procura manter estas últimas dentro das respectivas faixas. Observa-se ainda que a escolha de um $J_{K \lim }$ menor no caso 2 define um controlador cujo fator de excitação é reduzido mais rapidamente com o aumento do erro, resultando numa excitação menos agressiva e, conseqüentemente, em menor violação das faixas, tanto em freqüência quanto em amplitude.

Tabela 3.5 - Sintonia empregada nos testes do controlador do Problema (P4)

\begin{tabular}{|c|c|c|c|c|}
\hline \multicolumn{5}{|c|}{ Parâmetros fixos } \\
\hline Horizonte de predição $(n p)$ & 20 & \multirow{3}{*}{$\begin{array}{l}\text { Limites nas } \\
\text { manipuladas }\end{array}$} & $\boldsymbol{u}^{\max }$ & {$\left[\begin{array}{ll}15 & 15\end{array}\right]$} \\
\hline Horizonte de controle $(\mathrm{m})$ & 7 & & $\boldsymbol{u}^{\min }$ & {$\left[\begin{array}{ll}-10 & -10\end{array}\right]$} \\
\hline Set-point controladas $\left(\boldsymbol{y}^{s p}\right)$ & {$\left[\begin{array}{ll}0 & 0\end{array}\right]$} & & $\Delta \boldsymbol{u}^{\max }$ & {$\left[\begin{array}{ll}1 & 1\end{array}\right]$} \\
\hline Limite sup. das controladas $\left(y^{\max }\right)$ & {$\left[\begin{array}{ll}0.2 & 0.2\end{array}\right]$} & \multicolumn{2}{|c|}{ Peso das controladas $(\boldsymbol{Q})$} & {$\left[\begin{array}{ll}4 & 5\end{array}\right]$} \\
\hline Limite inf. das controladas $\left(y^{\mathrm{min}}\right)$ & {$\left[\begin{array}{ll}-0.2 & -0.2\end{array}\right]$} & \multicolumn{2}{|c|}{ Fator de supressão $(\boldsymbol{R})$} & {$\left[\begin{array}{ll}0.1 & 0.1\end{array}\right]$} \\
\hline \multicolumn{5}{|c|}{ Parâmetros variáveis } \\
\hline \multicolumn{2}{|l|}{ Parâmetro } & \multicolumn{2}{|c|}{ Caso 1} & Caso 2 \\
\hline \multicolumn{2}{|c|}{ Limite do erro $\left(\boldsymbol{e}_{\mathrm{lim}}\right) \mathrm{p} /$ cálculo do $J_{K \lim }$} & \multicolumn{2}{|c|}{$\left(\boldsymbol{y}^{\max }-\boldsymbol{y}^{\min }\right) / 1$} & $\left(\boldsymbol{y}^{\max }-\boldsymbol{y}^{\min }\right) / 2$ \\
\hline
\end{tabular}



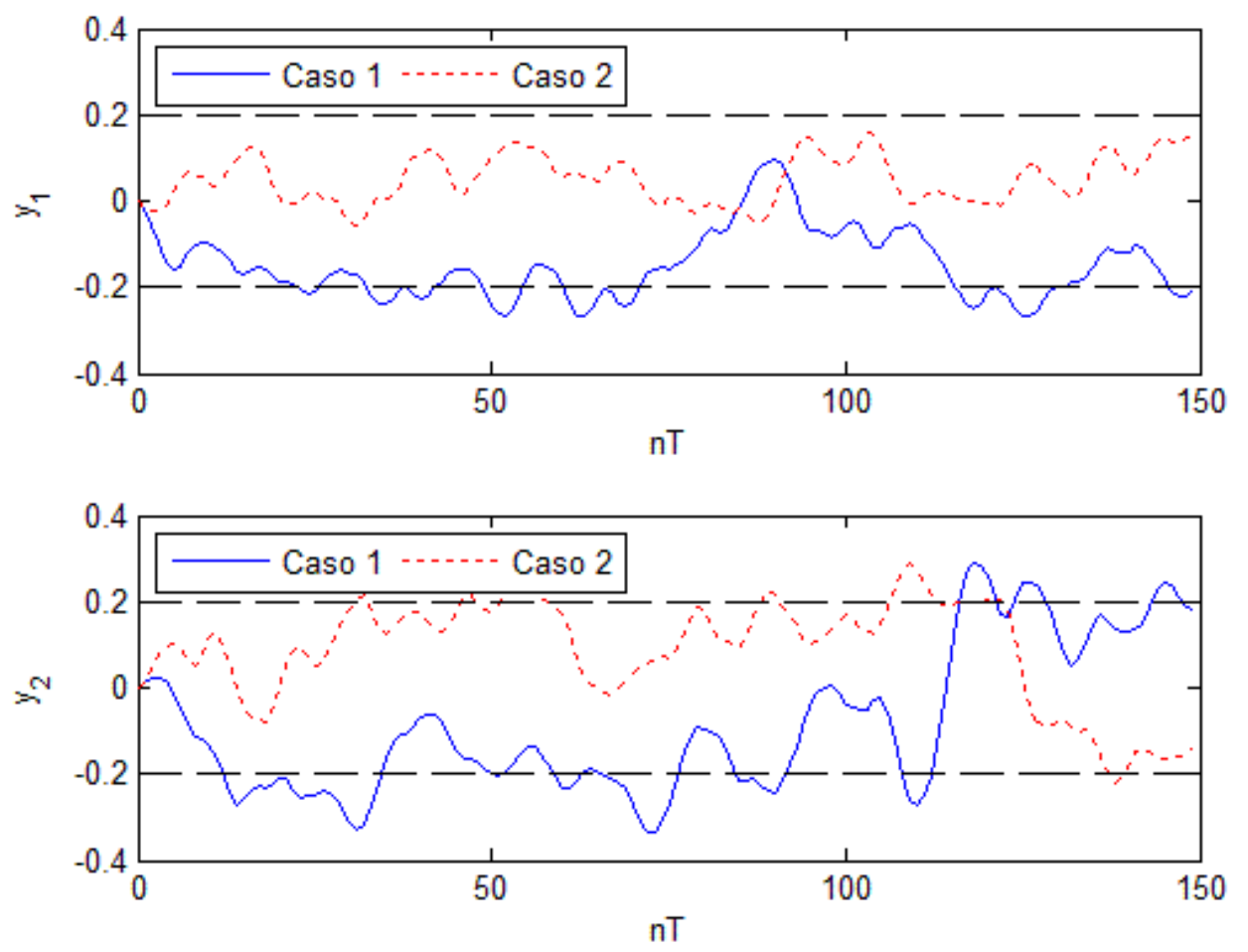

Figura 3.8 - Comportamento das variáveis controladas do controlador com restrição de excitação - Formulação (P4)
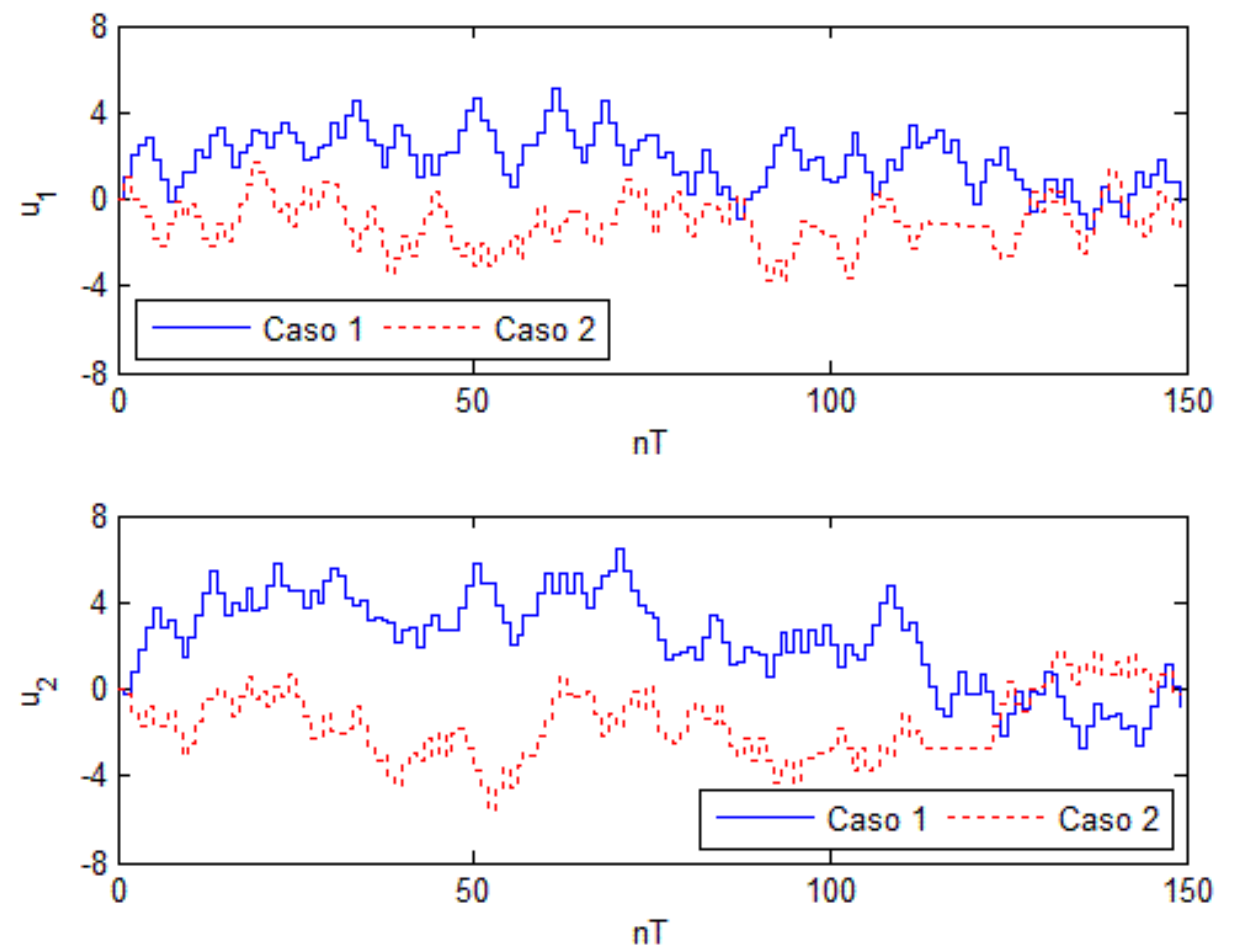

Figura 3.9 - Comportamento das variáveis manipuladas do controlador com restrição de excitação - Formulação (P4) 
Embora a observação das figuras 3.8 e 3.9 não evidencie a diferença da excitação produzida nos dois casos, a variação na excitação produzida na malha de controle de um caso para outro pode ser avaliada quantitativamente pela comparação das correspondentes variâncias dos sinais de entrada e saída indicadas na tabela 3.6. Como era de se esperar, a variância dos sinais no caso 1 é maior que no caso 2.

Tabela 3.6 - Variância dos sinais de entrada e saída para o controlador (P4)

\begin{tabular}{|c|c|c|c|c|}
\cline { 2 - 5 } \multicolumn{1}{c|}{} & \multicolumn{4}{c|}{ Variâncias } \\
\hline Caso & $y_{1}$ & $y_{2}$ & $u_{1}$ & $u_{2}$ \\
\hline Caso 1 & 0.0063 & 0.0288 & 1.6338 & 4.6611 \\
\hline Caso 2 & 0.0032 & 0.0151 & 1.2118 & 2.4425 \\
\hline
\end{tabular}

Outros exemplos com esta formulação são apresentados no capítulo 4 deste trabalho. 


\section{EXEMPLOS DE APLICAÇÕES}

Neste capítulo são apresentados exemplos da utilização do controlador MPC com restrição de excitação desenvolvido no capítulo anterior através da simulação de diversos casos em dois processos distintos da indústria de refino de petróleo. $\mathrm{Na}$ seção 4.1 os testes de identificação são aplicados a uma coluna debutanizadora utilizando as formulações dos problemas (P3) e (P4) para a geração dos dados e identificando modelos com estrutura ARX através do "toolbox" de identificação do Matlab. Na seção 4.2 os testes são aplicados a uma coluna de destilação C3/C4 e a identificação de modelos contínuos é executada segundo o procedimento proposto por Sotomayor; Odloak e Moro (2008), com o uso da ferramenta de identificação de sistemas em tempo contínuo (CONTSID toolbox, do inglês "Continuous-Time System IDentification" - GARNIER; GILSON; CERVELLIN, 2006).

\subsection{COLUNA DEBUTANIZADORA}

\subsubsection{Descrição do processo}

Nesta seção, os testes foram simulados considerando o processo estudado por Almeida Neto (1999), referente a uma coluna debutanizadora de uma unidade industrial de destilação da Refinaria Presidente Bernardes (RPBC) da Petrobras, cujo fluxograma está representado na figura 4.1.

De forma resumida, esta coluna tem como objetivo remover as frações instáveis presentes na corrente de carga (nafta instabilizada). Estas frações instáveis são incorporadas ao GLP (Gás Liquefeito de Petróleo) enquanto as frações estáveis formam a gasolina e a nafta petroquímica. 


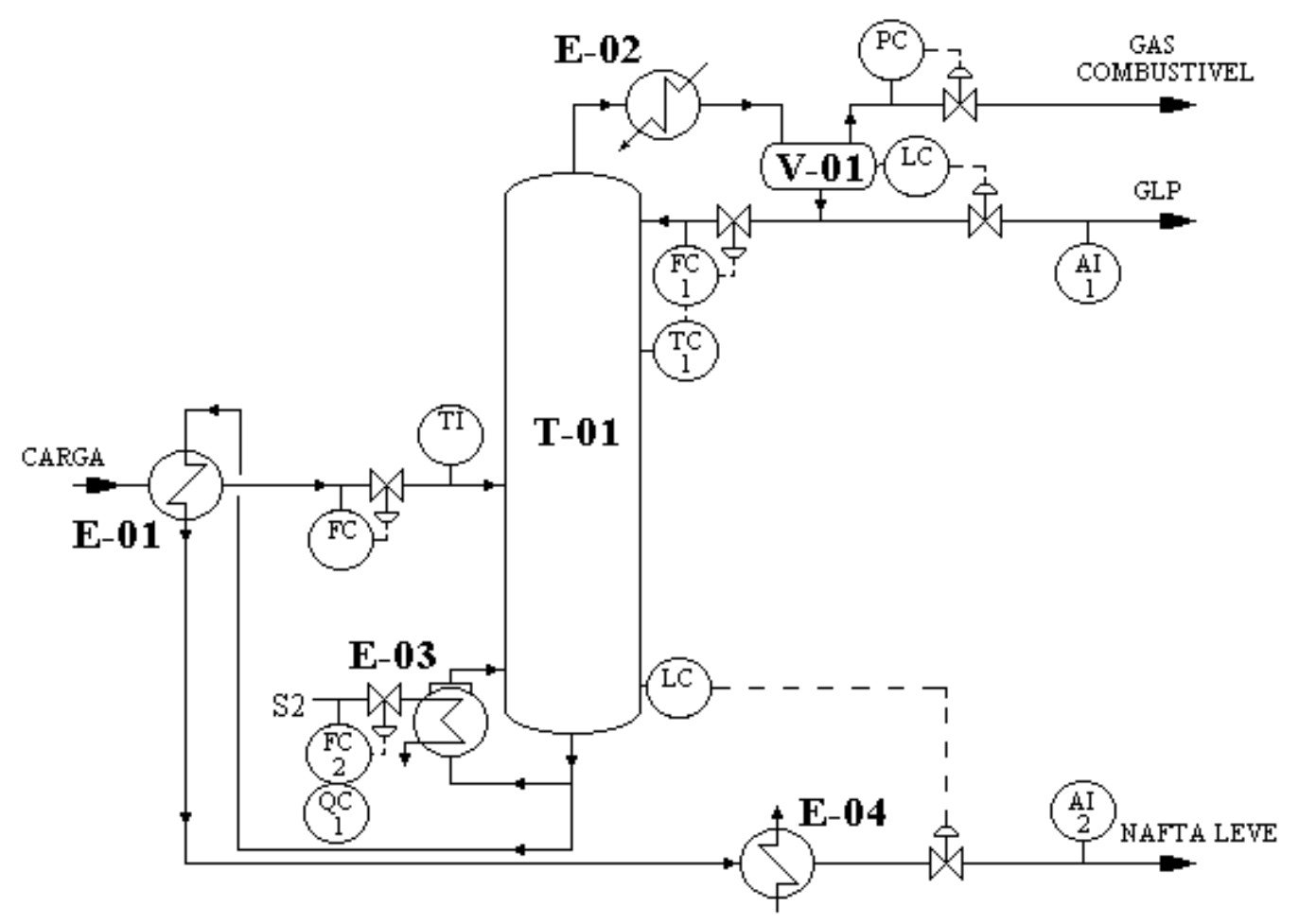

Figura 4.1 - Fluxograma de processo da coluna debutanizadora

O processo simulado tem duas variáveis controladas e duas variáveis manipuladas. A variável controlada $y_{1}$ representa o teor de componentes com mais de cinco carbonos (C5+ - \%) na corrente de GLP no topo da coluna e a variável $y_{2}$ a pressão de vapor Reid (PVR - kPa) da gasolina na corrente de fundo. As entradas manipuladas são a vazão de refluxo de topo, $u_{1}\left(\mathrm{~m}^{3} / \mathrm{d}\right)$, e a carga térmica do refervedor, $u_{2}$ (MMcal/h). Na verdade, o controlador preditivo fornece os "set-points" correspondentes às variáveis manipuladas $u_{1}$ e $u_{2}$ para os controladores FC-1 e QC-1 indicados na figura 4.1, respectivamente.

Para a realização das simulações foram selecionados os modelos correspondentes a três condições operacionais distintas, sendo ainda incluído um quarto modelo fictício, correspondente ao modelo 1 com ganhos reduzidos em 40\%. Os quatro modelos considerados estão indicados na tabela 4.1. 
Tabela 4.1 - Modelos da coluna debutanizadora usados nas simulações

\begin{tabular}{|c|c|c|c|}
\hline \multicolumn{2}{|c|}{ Modelo 1} & \multicolumn{2}{|c|}{ Modelo 2} \\
\hline \multicolumn{2}{|c|}{$\boldsymbol{G}_{1}(s)=$} & \multicolumn{2}{|c|}{$\boldsymbol{G}_{2}(s)=$} \\
\hline-0.2623 & 0.1368 & -0.3544 & 0.2044 \\
\hline$\overline{60 s^{2}+59.2 s+1}$ & $\overline{1164 s^{2}+99.7 s+1}$ & $\overline{218.6 s^{2}+50.1 s+1}$ & $\overline{1150 s^{2}+93.86 s+1}$ \\
\hline 0.1242 & -0.1351 & 0.0685 & -0.1256 \\
\hline$\overline{218.7 s^{2}+16.2 s+1}$ & $\overline{70 s^{2}+20 s+1}$ & $\overline{100.2 s^{2}+11.32 s+1}$ & $\overline{20 s^{2}+15 s+1}$ \\
\hline \multicolumn{2}{|c|}{ Modelo 3} & \multicolumn{2}{|c|}{ Modelo 4} \\
\hline \multicolumn{2}{|c|}{$\boldsymbol{G}_{3}(s)=$} & \multicolumn{2}{|c|}{$\boldsymbol{G}_{4}(s)=$} \\
\hline-0.2790 & 0.050 & -0.1574 & 0.0821 \\
\hline$\overline{59.77 s^{2}+99.61 s+1}$ & $\overline{499.8 s^{2}+73.77 s+1}$ & $\overline{60 s^{2}+59.2 s+1}$ & $\overline{1164 s^{2}+99.7 s+1}$ \\
\hline 0.1950 & -0.1722 & 0.0745 & -0.0811 \\
\hline$\overline{220.1 s^{2}+18.93 s+1}$ & $29.74 s^{2}+20.71 s+1$ & $\overline{218.7 s^{2}+16.2 s+1}$ & $\overline{70 s^{2}+20 s+1}$ \\
\hline
\end{tabular}

Todos os exemplos apresentados a seguir foram simulados no aplicativo Matlab ${ }^{\circledR}$. Os resultados dos testes de identificação apresentados também foram obtidos com o uso do "Identification Toolbox" do mesmo aplicativo.

\subsubsection{Influência do erro do modelo no desempenho do MPC}

Para verificar a influência do erro no modelo no desempenho do MPC, foram realizados alguns testes com o MPC normal (sem restrição de excitação). Nestes testes, o modelo considerado pelo controlador (nominal) é sempre o modelo 1 da tabela 4.1 enquanto o modelo da planta varia. O comportamento das variáveis controladas e manipuladas está ilustrado na figura 4.2.

Inicialmente, foi determinada uma sintonia que proporcionasse um desempenho satisfatório no caso nominal (modelo igual à planta) para uma variação em degrau no set-point das controladas (caso servo). Com esta mesma sintonia, foram simulados os casos onde o modelo da planta real considerado assumia cada um dos modelos apresentados na tabela 4.1. Os valores utilizados na sintonia empregada estão resumidos na tabela 4.2 . 
Tabela 4.2 - Sintonia empregada nos testes da figura 4.2

\begin{tabular}{|c|c|c|c|c|}
\hline Horizonte de predição $(n p)$ & 20 & \multicolumn{2}{|c|}{ Set-point das controladas $\left(y^{s p}\right)$} & {$\left[\begin{array}{ll}1 & -1\end{array}\right]$} \\
\hline Horizonte de controle $(m)$ & 7 & \multirow{3}{*}{$\begin{array}{l}\text { Limites nas } \\
\text { manipuladas }\end{array}$} & $u^{\max }$ & {$\left[\begin{array}{ll}15 & 15\end{array}\right]$} \\
\hline Peso das controladas $(\boldsymbol{Q})$ & {$\left[\begin{array}{ll}1 & 1\end{array}\right]$} & & $u^{\min }$ & {$\left[\begin{array}{ll}-10 & -10\end{array}\right]$} \\
\hline Fator de supressão $(\boldsymbol{R})$ & {$\left[\begin{array}{ll}0.1 & 0.1\end{array}\right]$} & & $\Delta \boldsymbol{u}^{\max }$ & {$\left[\begin{array}{ll}1 & 1\end{array}\right]$} \\
\hline
\end{tabular}
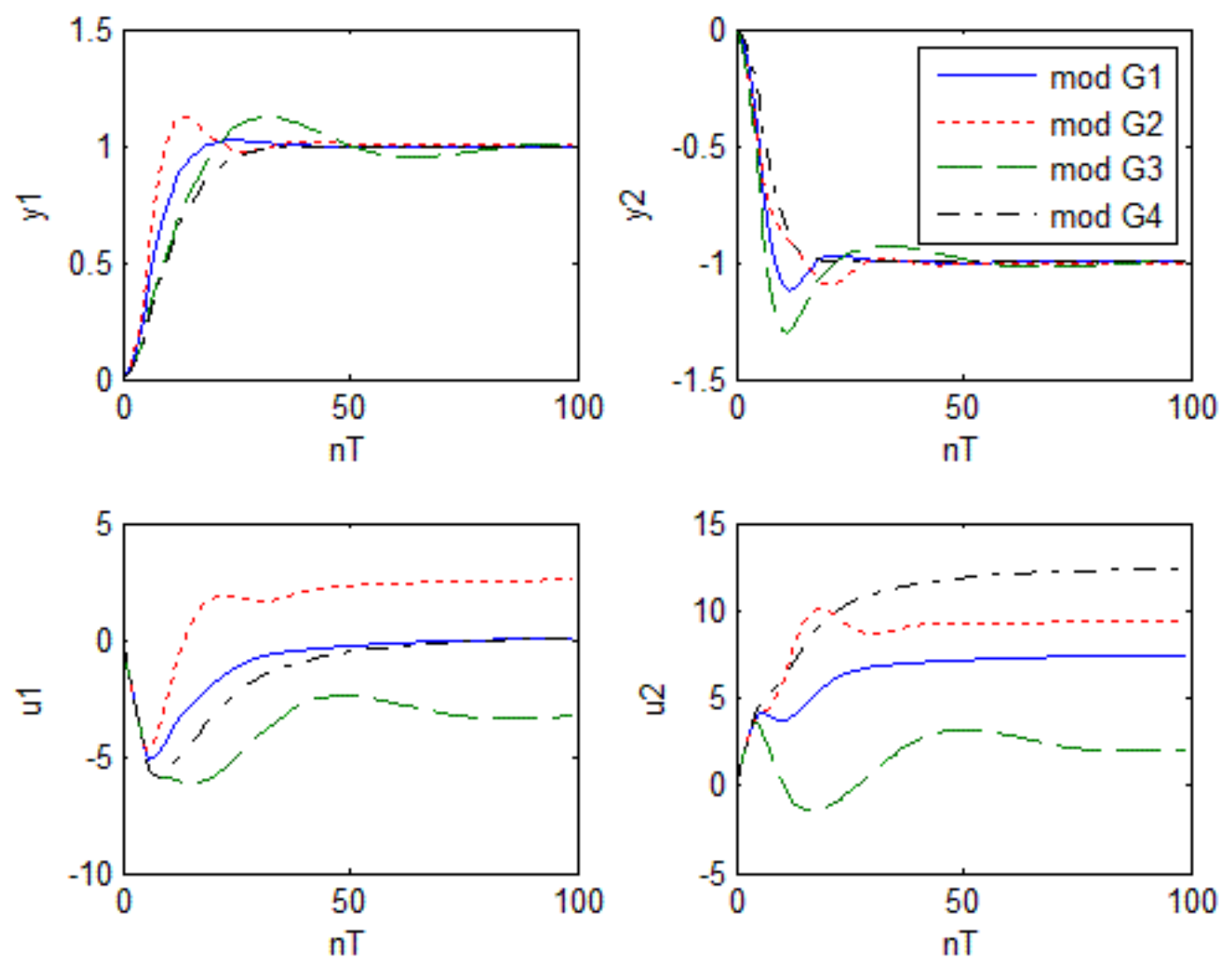

Figura 4.2 - Influência do erro do modelo no desempenho do MPC

A figura 4.2 comprova que o erro no modelo usado pelo MPC para as predições tem grande influência no comportamento das variáveis e no desempenho do sistema. $O$ pior caso observado corresponde à planta com modelo $\mathrm{G} 3$, onde o tempo de estabilização praticamente dobrou e a variável $y_{2}$ apresentou um "overshoot" significativo em relação ao caso nominal. Outro aspecto a se observar é que, embora o controlador tenha conseguido levar as variáveis controladas aos respectivos "set-points" em todos os casos, existe uma diferença significativa nos valores de regime permanente das variáveis manipuladas, decorrente das diferenças de ganhos entre os diversos modelos. Neste teste, onde propositalmente se abriu os limites das entradas, isto não teve maiores conseqüências, porém num cenário onde 
estas entradas tivessem limites mais restritos, certamente se observaria a saturação de alguma entrada e o aparecimento de erro de regime ("off-set") em alguma variável controlada.

\subsubsection{Controlador com restrição de excitação - caso nominal}

Nesta seção, foi simulado o caso nominal (modelo igual à planta) com a mesma sintonia empregada na seção 4.1.2, porém utilizando o controlador com restrição de excitação e formulação conforme apresentado na seção 3.5 - Problema (P3). Apenas para permitir a comparação com os resultados da seção anterior, foi também considerado o caso servo para a mesma variação no "set-point" das controladas. O comportamento das variáveis está apresentado na figura 4.3.
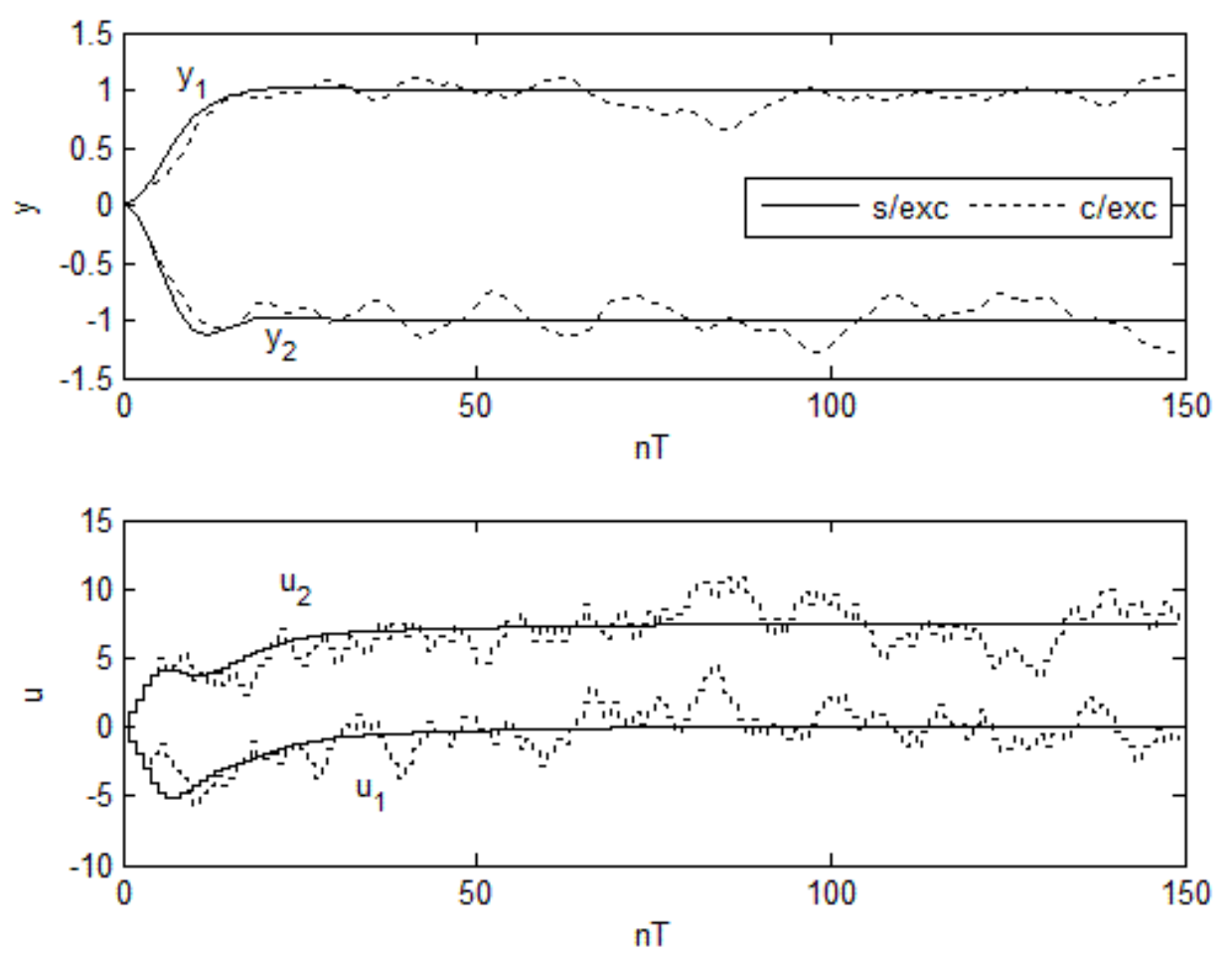

Figura 4.3 - Controlador MPC convencional e com restrição de excitação

Como se pode observar, a restrição de excitação introduzida faz com que as variáveis manipuladas e controladas oscilem próximo aos valores do MPC normal. O rank da pseudo-matriz de informação utilizado neste exemplo foi igual a seis. 
Aqui se pretendia apenas ilustrar as variações produzidas pela restrição de excitação nas variáveis controladas e manipuladas. Adiante, será realizado teste de identificação com um número bem maior de pontos para verificar se é possível a identificação do modelo da planta.

\subsubsection{Teste de identificação - caso 1 - caso nominal}

Neste teste, o objetivo foi aplicar técnicas de identificação para verificar se os dados produzidos permitem a correta identificação do modelo da planta. Considerou-se o caso nominal (modelo igual à planta) e se utilizou o controlador com restrição de excitação na formulação apresentada no Problema (P3).

Inicialmente se verificou se o período de amostragem que vinha sendo empregado no controlador ( $T$ igual a 5 unidades de tempo) atendia as regras práticas apresentadas na seção 2.1.3. Como a princípio já se conhece o modelo nominal programado no controlador, foram levantadas as características de interesse deste modelo, apresentadas na tabela 4.3.

Tabela 4.3 - Características dinâmicas do modelo $G_{1}(s)$

\begin{tabular}{|l|c|c|c|c|}
\cline { 2 - 4 } \multicolumn{1}{c|}{} & $G_{1}(1,1)$ & $G_{1}(1,2)$ & $G_{1}(2,1)$ & $G_{1}(2,2)$ \\
\cline { 2 - 5 } \multicolumn{1}{c|}{} & $y_{1} \times u_{1}$ & $y_{1} \times u_{2}$ & $y_{2} \times u_{1}$ & $y_{2} \times u_{2}$ \\
\hline Constante de tempo dominante $\left(\tau_{d}-\min \right)$ & 58.1 & 86.2 & 27.0 & 15.5 \\
\hline Tempo de estabilização $2 \%\left(T_{s}-\min \right)$ & 229 & 352 & 86 & 66 \\
\hline
\end{tabular}

Como se pode constatar, o período de 5 unidades de tempo (5 minutos) utilizado no controlador é inferior às duas recomendações práticas citadas em 2.1.3. Assim, pode-se a princípio considerar tal período de amostragem adequado.

Atendendo à recomendação de que a duração mínima do experimento deva ser maior que 10 vezes o maior tempo de estabilização, foram coletados 1500 pontos das entradas e saídas, apresentados nas figuras 4.4 e 4.5 . 

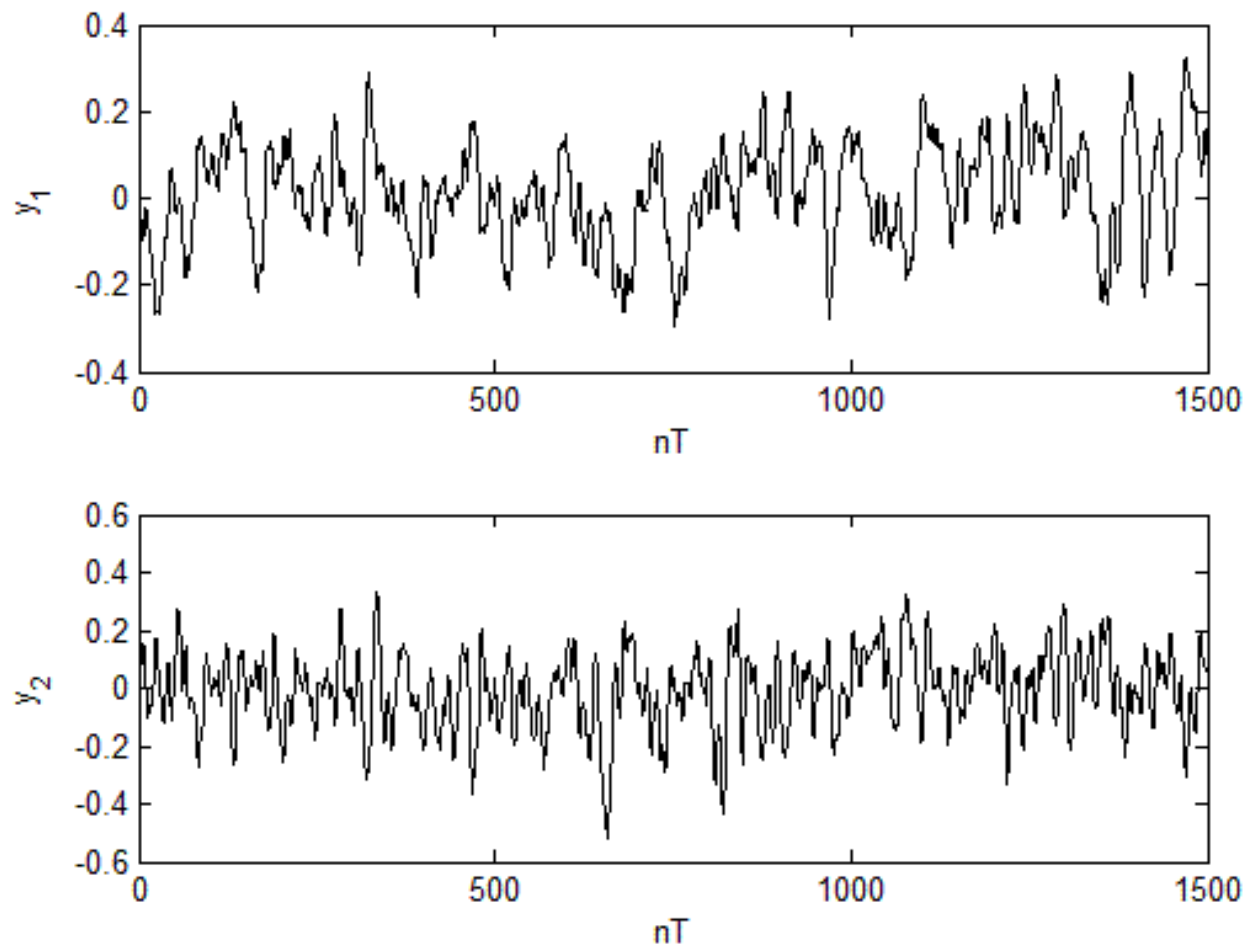

Figura 4.4 - Variáveis controladas da coluna debutanizadora - caso nominal
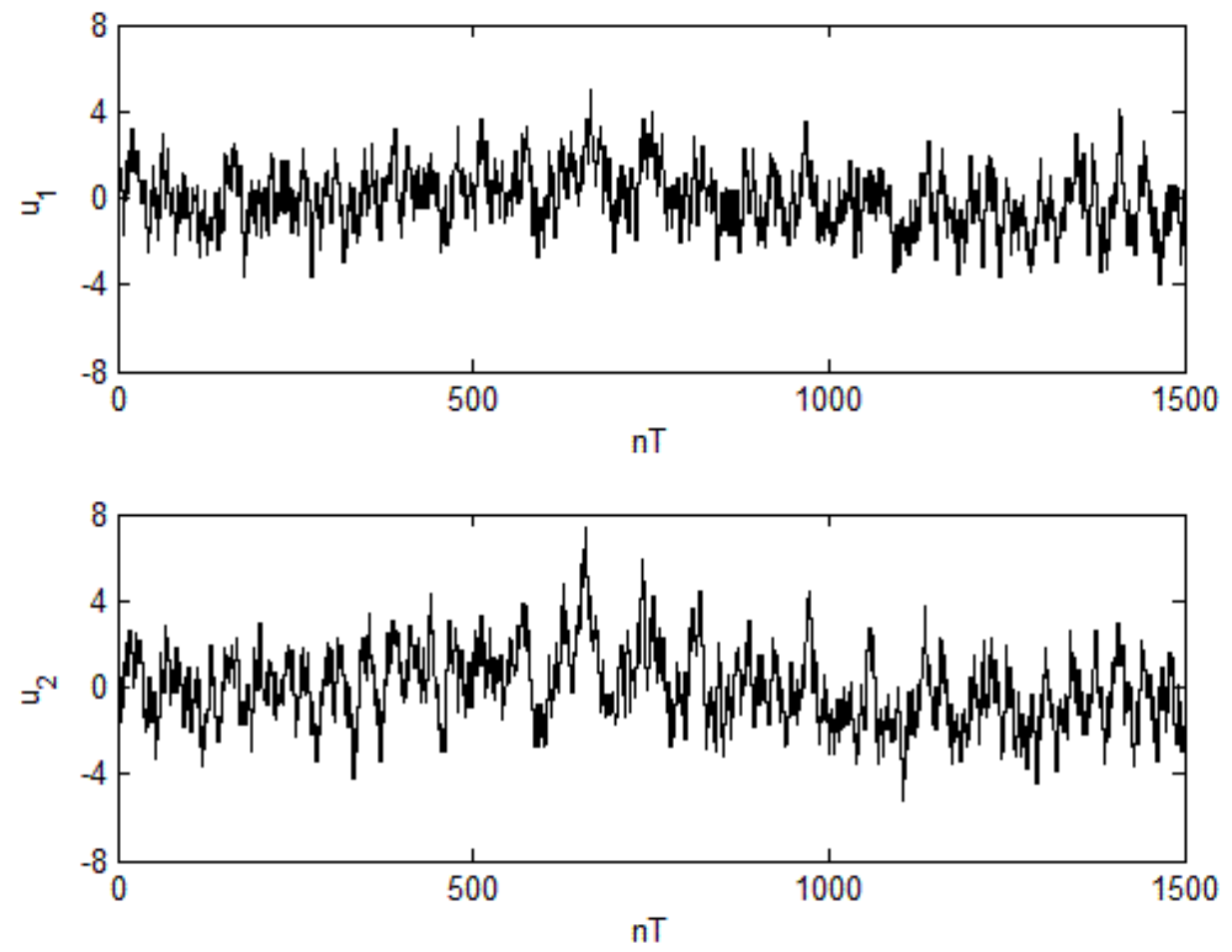

Figura 4.5 - Variáveis manipuladas da coluna debutanizadora - caso nominal

Estes pontos foram então divididos em dois conjuntos de dados: um com 1000 pontos, a ser usado na identificação e outro com 500 pontos, a ser usado na 
validação cruzada do modelo obtido. Todos os testes de identificação foram realizados empregando-se a interface gráfica (GUI) do "Identification Toolbox" do Matlab.

Em seguida, foram executados os testes de identificação do modelo ARX que melhor se ajustasse aos dados separados para este fim. A escolha das ordens do modelo ARX foi feita por tentativa e erro, embora seja possível se utilizar rotinas automatizadas para busca do tempo morto e das ordens que fornecem o melhor ajuste, como no trabalho de Miranda (2005). A tabela 4.4 apresenta os valores do critério de informação de Akaike (AIC), da função-perda e do erro final de predição (FPE) para cada seleção:

Tabela 4.4 - Busca da ordem do modelo ARX

\begin{tabular}{|c|c|c|c|c|c|c|}
\hline Caso & $\mathrm{na}$ & $\mathrm{nb}$ & $\mathrm{nk}$ & AIC & Loss Func & FPE \\
\hline $\mathrm{a}$ & 2 & 2 & 1 & -23.1581 & $8.576 \mathrm{e}-011$ & $8.761 \mathrm{e}-011$ \\
\hline $\mathrm{b}$ & 3 & 3 & 1 & -35.4160 & $4.028 \mathrm{e}-016$ & $4.159 \mathrm{e}-016$ \\
\hline $\mathrm{c}$ & 4 & 4 & 1 & -133.9070 & $6.705 \mathrm{e}-059$ & $6.998 \mathrm{e}-059$ \\
\hline
\end{tabular}

Como a escolha de ordens maiores que as indicadas na tabela não proporcionou redução significativa no AIC, optou-se por utilizar os parâmetros da última linha da tabela 4.4, para não se incorrer em sobre-parametrização do modelo.

$\mathrm{Na}$ verdade, as ordens com que se obteve o melhor ajuste ([na,nb,nk]=[4,4,1]) tem uma justificativa teórica pois, de posse do modelo nominal teórico $G_{1}(s)$ no domínio da freqüência, pode-se obter o modelo teórico discretizado com um período de amostragem de 5 unidades de tempo indicado na eq.(4.1).

$$
\boldsymbol{G}_{1}(z)=\left[\begin{array}{cc}
\frac{-0.01730 z^{-1}-0.004138 z^{-2}}{1-0.9255 z^{-1}+0.007202 z^{-2}} & \frac{0.001278 z^{-1}+0.001108 z^{-2}}{1-1.6340 z^{-1}+0.6516 z^{-2}} \\
\frac{0.006240 z^{-1}+0.005513 z^{-2}}{1-1.5960 z^{-1}+0.6905 z^{-2}} & \frac{-0.01538 z^{-1}-0.009569 z^{-2}}{1-1.05960 z^{-1}+0.6905 z^{-2}}
\end{array}\right]
$$

Sabendo que $\boldsymbol{y}(z)=\boldsymbol{G}_{1}(z) \boldsymbol{u}(z)$, tem-se:

$$
y_{1}(z)=G_{1,1}(z) u_{1}(z)+G_{1,2}(z) u_{2}(z)
$$




$$
y_{1}(z)=\frac{-0.01730 z^{-1}-0.004138 z^{-2}}{1-0.9255 z^{-1}+0.007202 z^{-2}} u_{1}(z)+\frac{0.001278 z^{-1}+0.001108 z^{-2}}{1-1.6340 z^{-1}+0.6516 z^{-2}} u_{2}(z)
$$

Como o modelo ARX considera um denominador único para cada saída:

$$
\begin{aligned}
y_{1}(z)= & \frac{-0.0173 z^{-1}+0.0241 z^{-2}-0.0045 z^{-3}-0.0027 z^{-4}}{1-2.5597 z^{-1}+2.1713 z^{-2}-0.6149 z^{-3}+0.0047 z^{-4}} u_{1}(z)+ \\
& +\frac{0.0013 z^{-1}-0.0001 z^{-2}-0.0010 z^{-3}}{1-2.5597 z^{-1}+2.1713 z^{-2}-0.6149 z^{-3}+0.0047 z^{-4}} u_{2}(z)
\end{aligned}
$$

Ou na equivalente equação no domínio do tempo:

$$
\begin{aligned}
& y_{1}(k)-2.5597 y_{1}(k-1)+2.1713 y_{1}(k-2)-0.6149 y_{1}(k-3)+0.0047 y_{1}(k-4)= \\
& =-0.0173 u_{1}(k-1)+0.0241 u_{1}(k-2)-0.0045 u_{1}(k-3)-0.0027 u_{1}(k-4)+ \\
& +0.0013 u_{2}(k-1)-0.0001 u_{2}(k-2)-0.0010 u_{2}(k-3)+0 u_{2}(k-4)
\end{aligned}
$$

De forma análoga para a segunda variável controlada:

$$
\begin{aligned}
& y_{2}(k)-2.6508 y_{2}(k-1)+2.6137 y_{2}(k-2)-1.1109 y_{2}(k-3)+0.1655 y_{2}(k-4)= \\
& =0.0062 u_{1}(k-1)-0.0011 u_{1}(k-2)-0.0043 u_{1}(k-3)+0.0013 u_{1}(k-4)- \\
& -0.0154 u_{2}(k-1)+0.0150 u_{2}(k-2)+0.0046 u_{2}(k-3)-0.0066 u_{2}(k-4)
\end{aligned}
$$

Finalmente, escrevendo-se as eq.(4.4) e (4.5) na forma de um modelo ARX multivariável, tem-se:

$$
\begin{aligned}
& \boldsymbol{y}(k)+\left[\begin{array}{cc}
-2.5597 & 0 \\
0 & -2.6508
\end{array}\right] \boldsymbol{y}(k-1)+\left[\begin{array}{cc}
2.1713 & 0 \\
0 & 2.6137
\end{array}\right] \boldsymbol{y}(k-2) \\
& +\left[\begin{array}{cc}
-0.6149 & 0 \\
0 & -1.1109
\end{array}\right] \boldsymbol{y}(k-3)+\left[\begin{array}{cc}
0.0047 & 0 \\
0 & 0.1655
\end{array}\right] \boldsymbol{y}(k-4)= \\
& =\left[\begin{array}{cc}
-0.0173 & 0.0013 \\
0.0062 & -0.0154
\end{array}\right] \boldsymbol{u}(k-1)+\left[\begin{array}{cc}
+0.0241 & -0.0001 \\
-0.0011 & 0.0150
\end{array}\right] \boldsymbol{u}(k-2)+ \\
& +\left[\begin{array}{cc}
-0.0045 & -0.0010 \\
-0.0043 & 0.0046
\end{array}\right] \boldsymbol{u}(k-3)+\left[\begin{array}{cc}
-0.0027 & 0 \\
0.0013 & -0.0066
\end{array}\right] \boldsymbol{u}(k-4)
\end{aligned}
$$

$\mathrm{Na}$ eq.(4.6) observa-se que as ordens do modelo nominal (teórico) são na=4, nb=4 e nk=1, o que está de acordo com o melhor ajuste obtido no caso c da tabela 4.4. 
O modelo identificado no Matlab foi bastante próximo do modelo teórico, conforme se constata comparando-se as matrizes da eq.(4.6) com a saída do programa Matlab listada abaixo:

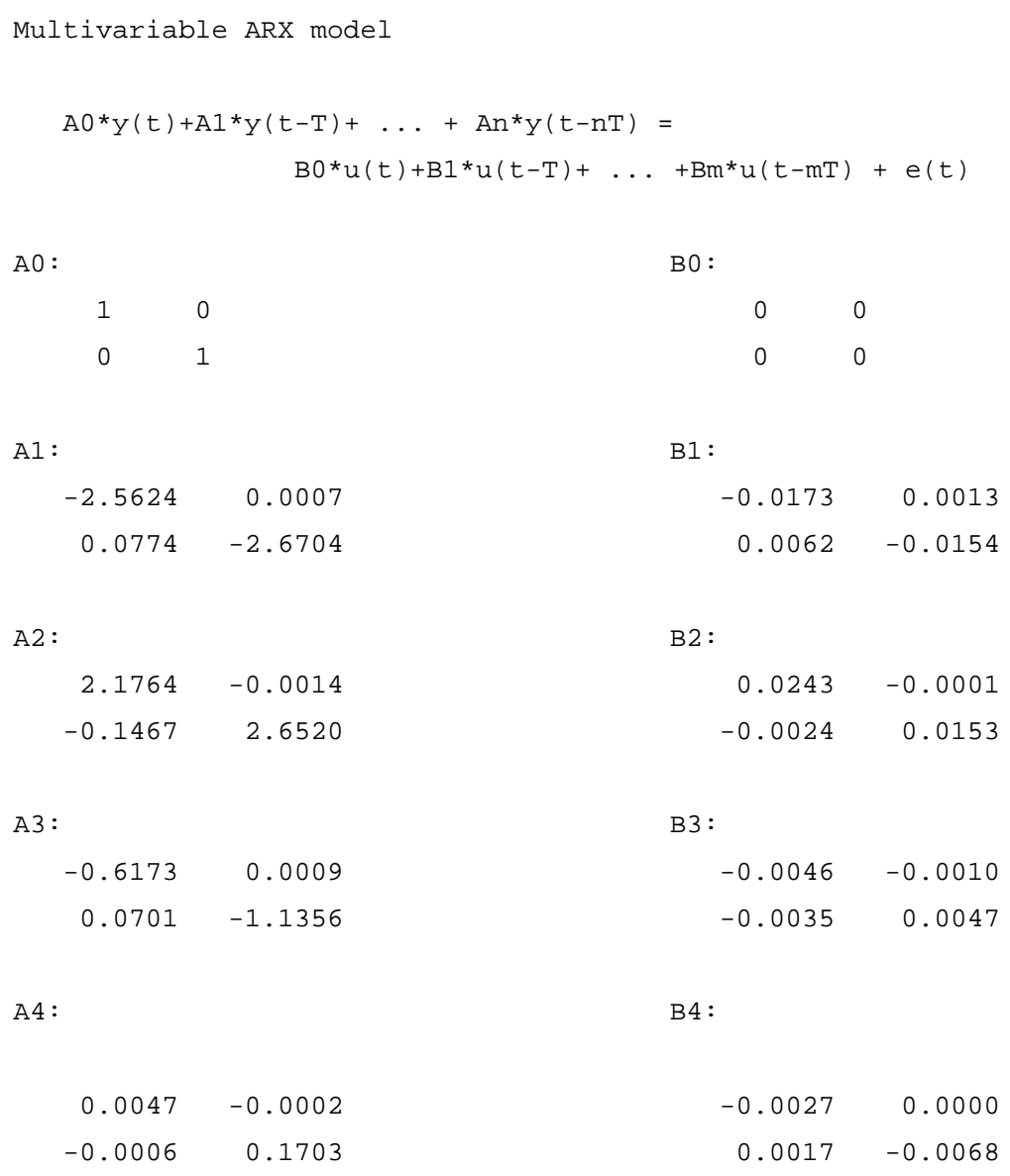

A๑:

10

A1:

$-2.5624 \quad 0.0007$

$0.0774-2.6704$

A2:

$2.1764-0.0014$

$-0.1467 \quad 2.6520$

A3:

$-0.6173 \quad 0.0009$

$0.0701-1.1356$

A4 :
0.0047
$-\odot .0002$
$-0.0006$
0.1703

BO :

$\odot \quad \odot$

B1:
$-0.0173$
0.0013
$-0.0154$

0.0062

B2:

$0.0243 \quad-0.0001$

B3:

$-0.0046-0.0010$

$\begin{array}{lll}-0.0035 & 0.0047\end{array}$

B4:
$-0.0027$
0.0000
0.0017
$-0.0068$

Observou-se apenas uma pequena correlação entre as saídas, presente nas matrizes $A 1, A 2$ e $A 3$ do modelo identificado e inexistente no modelo teórico correspondente à planta real.

A figura 4.6 apresenta o ajuste da resposta do modelo identificado ao conjunto de dados usado na validação cruzada, enquanto a figura 4.7 apresenta a comparação entre as respostas ao degrau do modelo identificado e da planta real. Embora esta comparação não seja possível em um caso real, quando não se conhece a priori o modelo da planta, é uma boa opção de avaliação em casos simulados como o atual. 

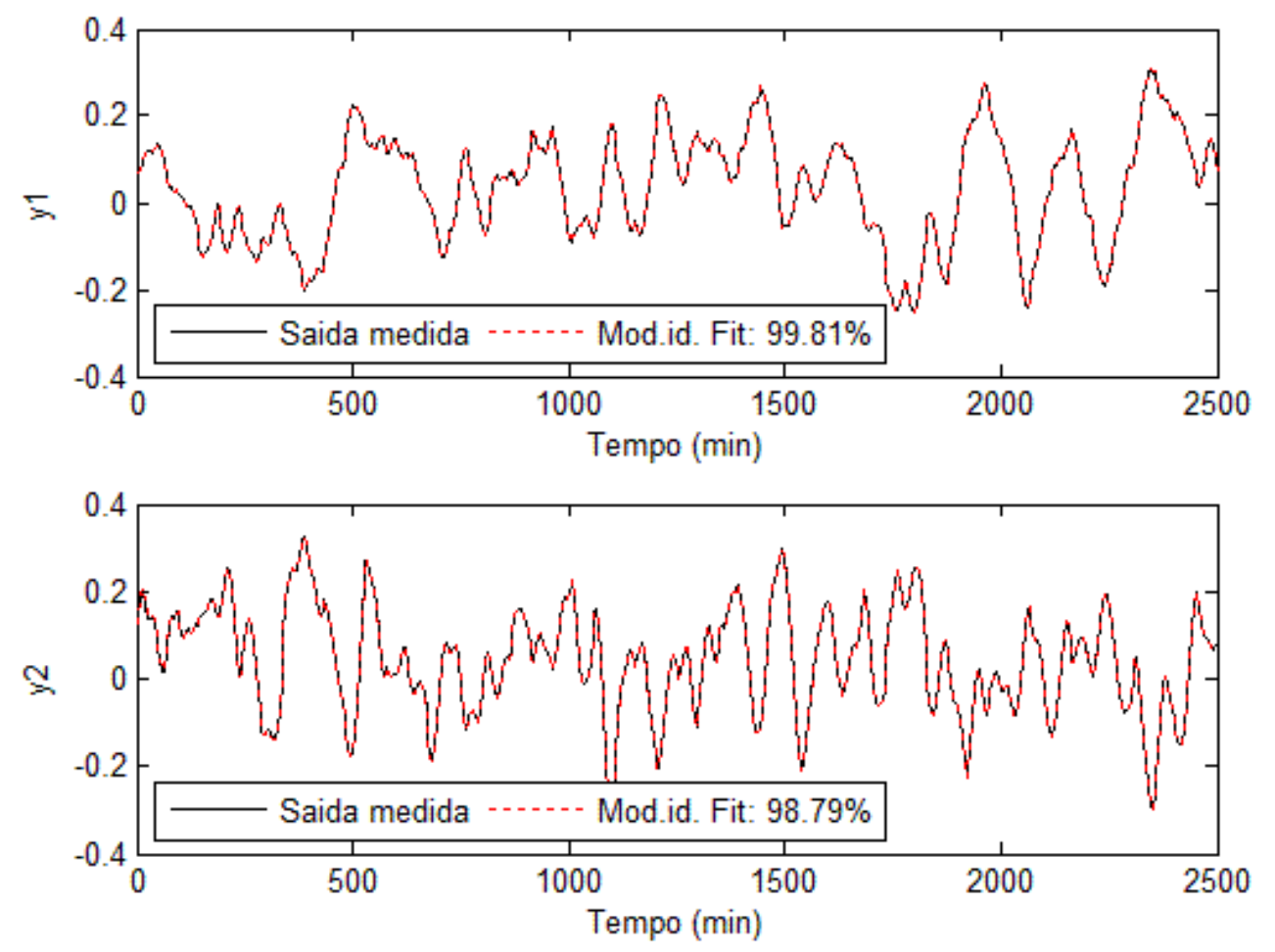

Figura 4.6 - Validação cruzada do modelo identificado - caso 1
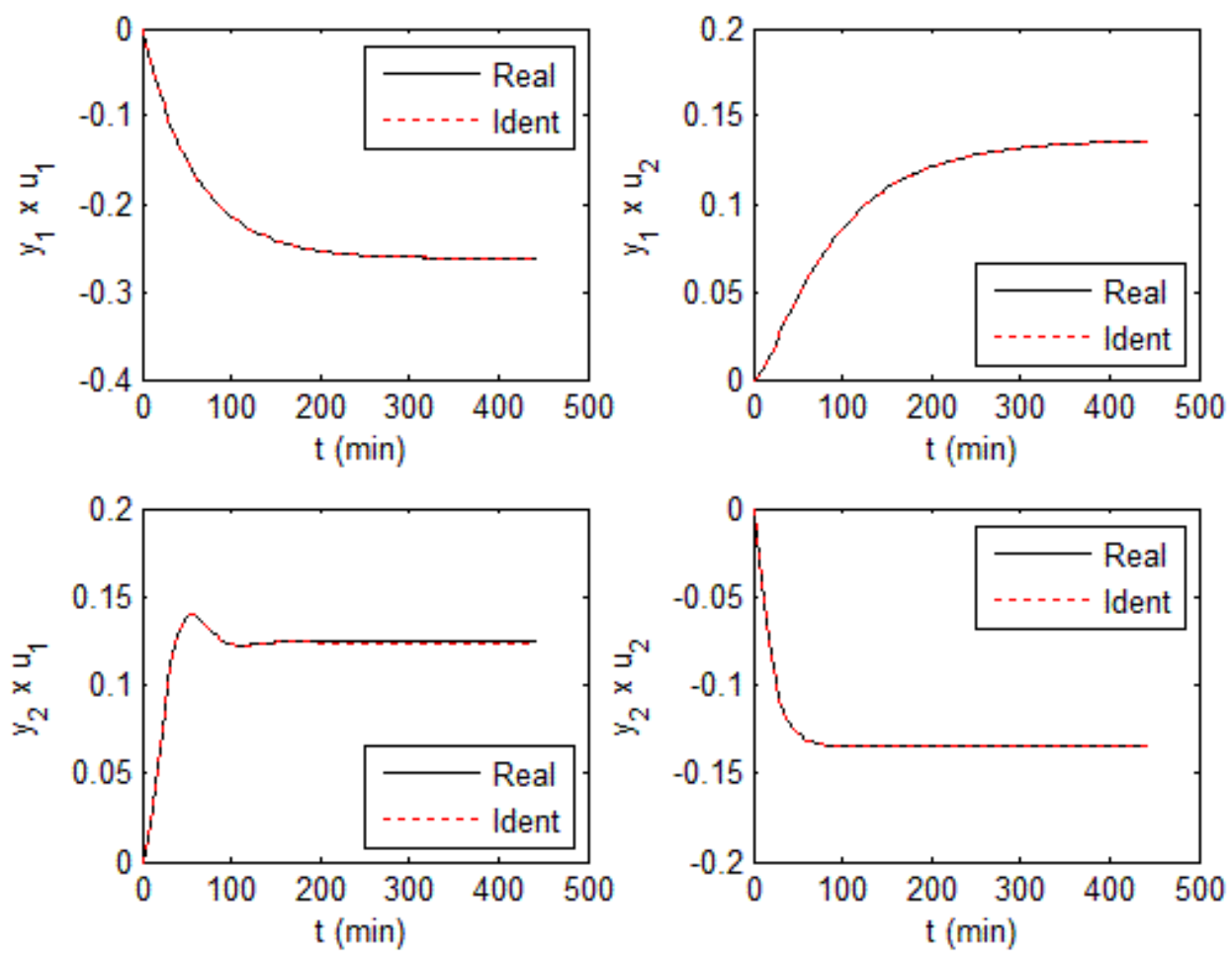

Figura 4.7 - Respostas ao degrau da planta e do modelo identificado - caso 1

Nas duas técnicas de validação usadas observou-se bom comportamento do modelo identificado. Na figura 4.6 a validação cruzada evidenciou o bom ajuste da resposta 
do modelo ao conjunto de dados reservado para validação enquanto na figura 4.7 se observa que as respostas ao degrau dos modelos teórico (real) e identificado são praticamente coincidentes, o que valida o teste de identificação em estudo.

\subsubsection{Teste de identificação - caso 2 - modelo diferente da planta}

Neste caso foi simulado o controlador com restrição de excitação, Problema (P3), para o caso onde o modelo nominal utilizado para as predições do controlador foi o modelo 1 da tabela 4.1 enquanto a planta real foi representada pelo modelo 3 da mesma tabela. A intenção foi verificar se o mesmo procedimento usado na seção anterior possibilita efetivamente a identificação da planta, pois se suspeitava que eventualmente pudesse ser identificado o modelo nominal configurado no MPC.

As figuras 4.8 e 4.9 mostram o conjunto de dados utilizado neste teste. Uma primeira observação é que este conjunto apresenta uma variabilidade maior nas entradas e saídas em comparação com o caso nominal anterior.
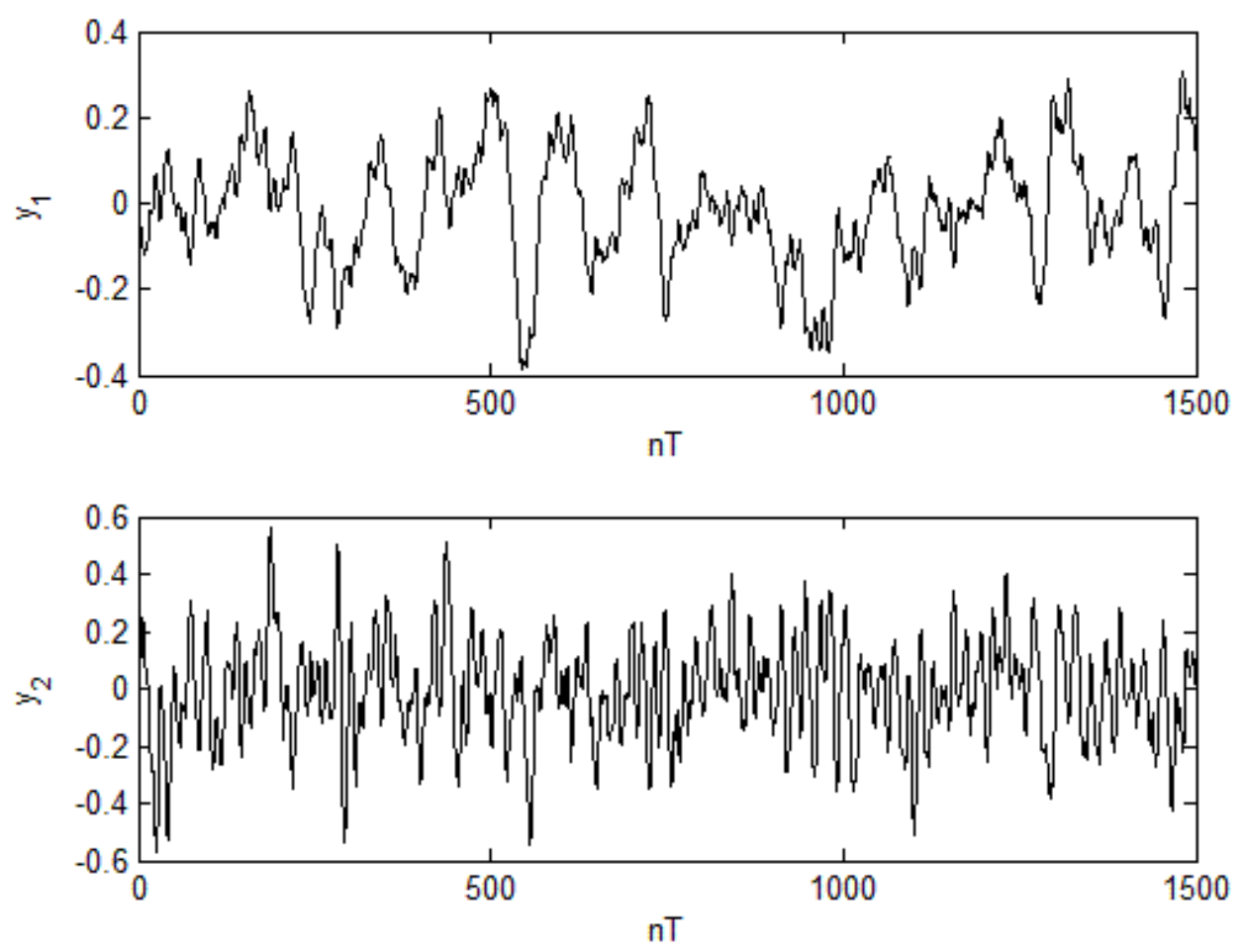

Figura 4.8 - Variáveis controladas da coluna debutanizadora - caso 2 

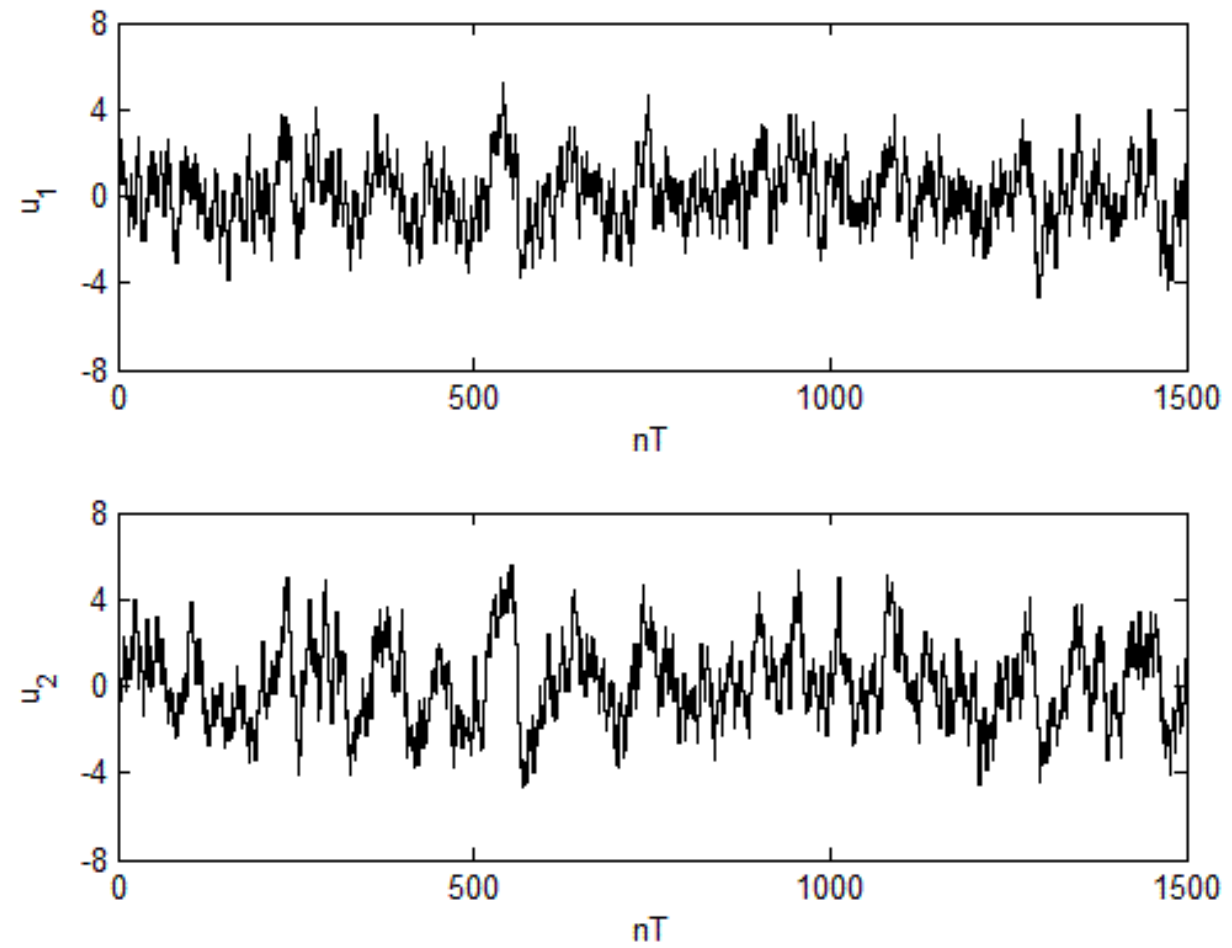

Figura 4.9 - Variáveis manipuladas da coluna debutanizadora - caso 2

Da mesma forma que na seção anterior, foram coletados 1500 pontos de cada variável e separados em dois conjuntos de dados: um para identificação, com 1000 pontos, e outro para validação cruzada, com 500 pontos.

A figura 4.10 apresenta a validação cruzada do modelo obtido. Na mesma figura foi incluída também a resposta do modelo nominal, para fins de comparação

Na figura 4.11 são apresentadas as respostas ao degrau do modelo real da planta, do modelo identificado e do modelo nominal. As ordens usadas no modelo ARX foram as mesmas da seção anterior ([na,nb,nk] $=[4,4,1])$. Como no caso anterior, as respostas dos modelos real e identificado são praticamente coincidentes e bastante diferentes das respostas do modelo nominal, principalmente para os pares $\left(y_{1} \times u_{2}\right)$, $\left(y_{2} \times u_{1}\right)$ e $\left(y_{2} \times u_{2}\right)$. 

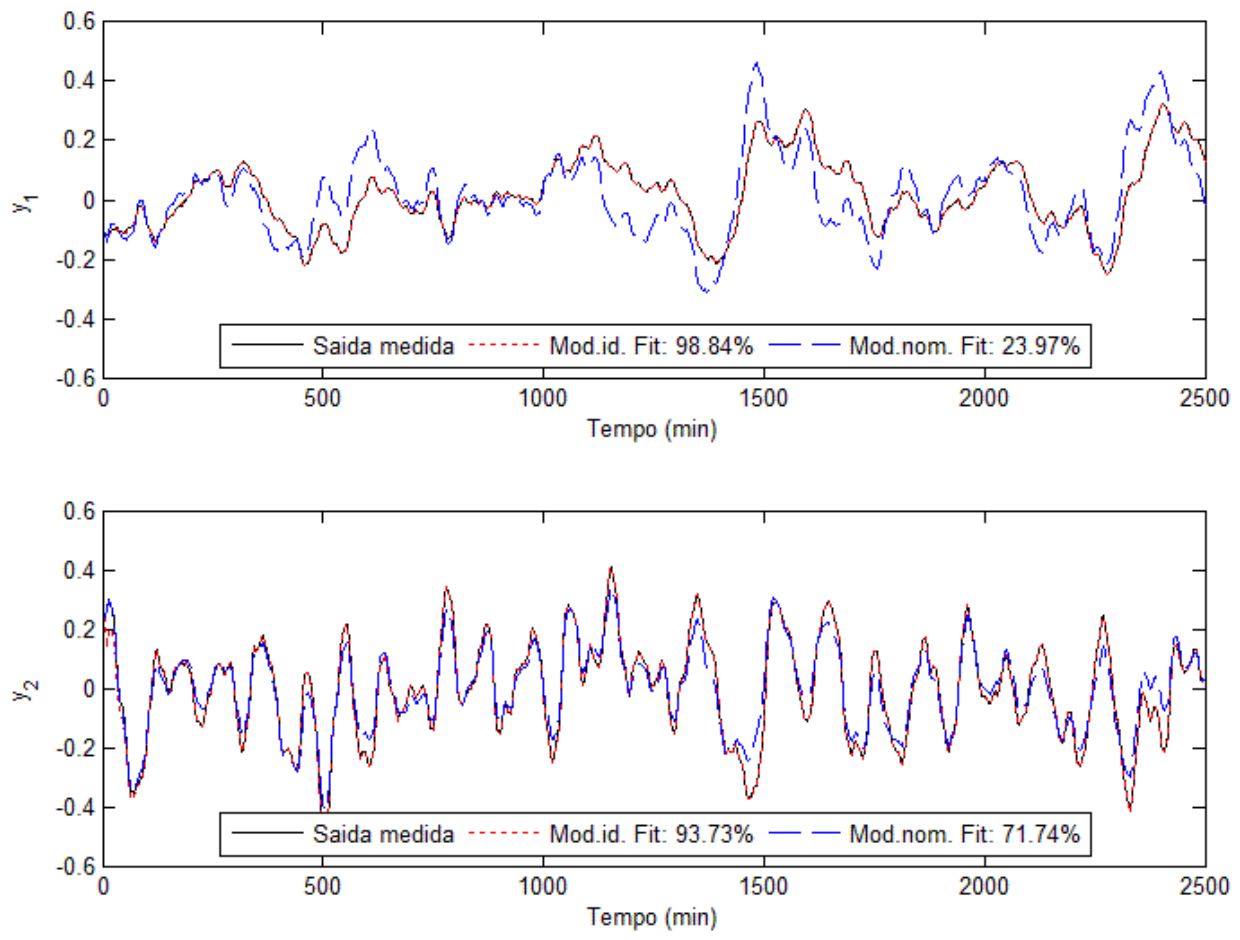

Figura 4.10 - Validação cruzada do modelo identificado - caso 2
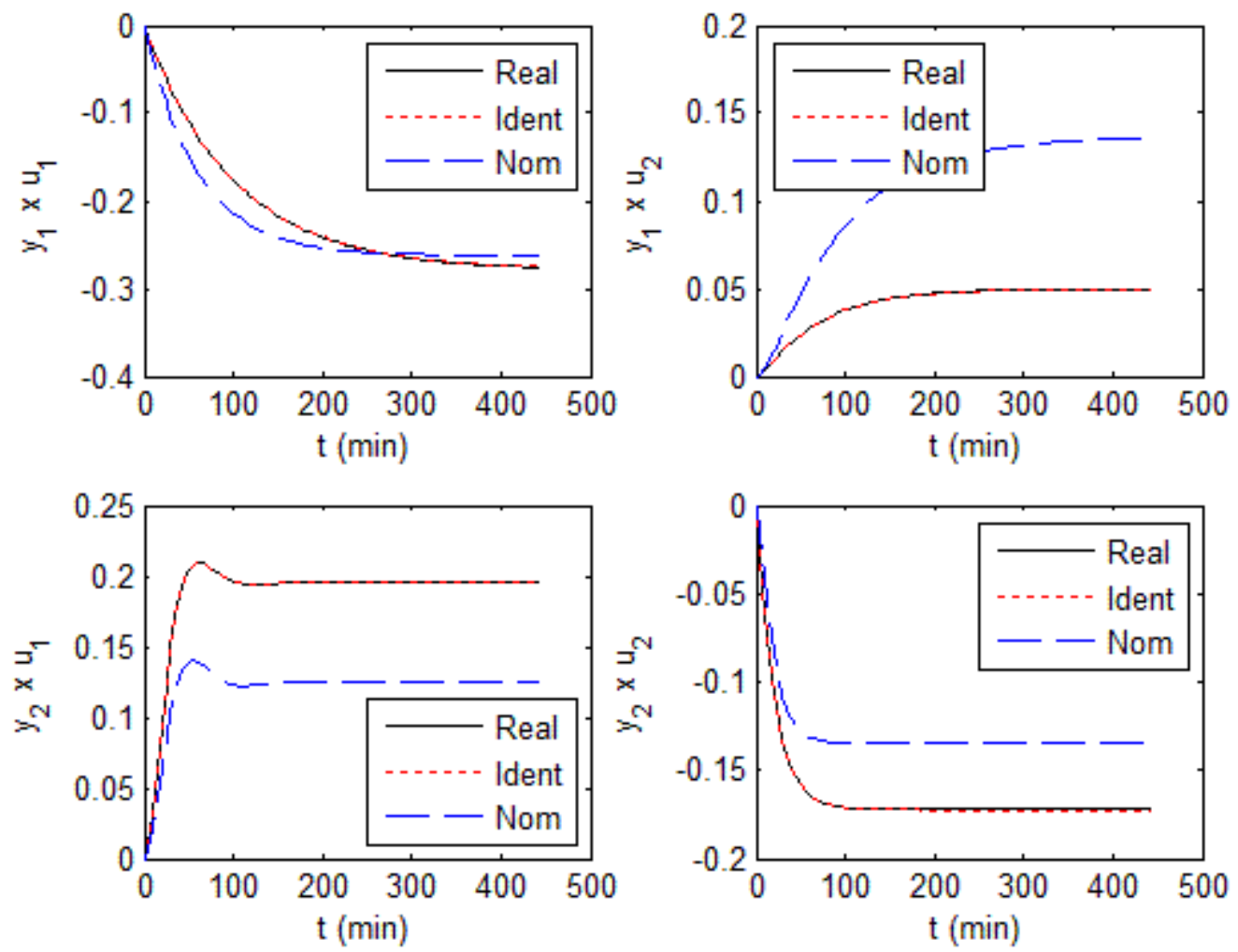

Figura 4.11 - Respostas ao degrau da planta, do modelo identificado e do modelo nominal - caso 2 
Observa-se na figura 4.10 que o modelo identificado se ajusta muito bem às saídas medidas dos dados de validação, enquanto o modelo nominal, que está sendo usado pelo MPC para as predições, diverge bastante dos dados medidos, principalmente para a variável $y_{1}$.

\subsubsection{Teste de identificação - caso 3 - planta com ruído}

Neste teste foi considerada a presença de ruído branco aditivo com variância de $2.25 \times 10^{-4}$ nas variáveis medidas, com o objetivo de verificar sua influência no desempenho do controlador e na qualidade da identificação. Na figura 4.12 está representada a resposta do controlador no modo servo, sem excitação e com ruído nas controladas, apenas para se ter uma idéia do nível de ruído em relação às variações das controladas.
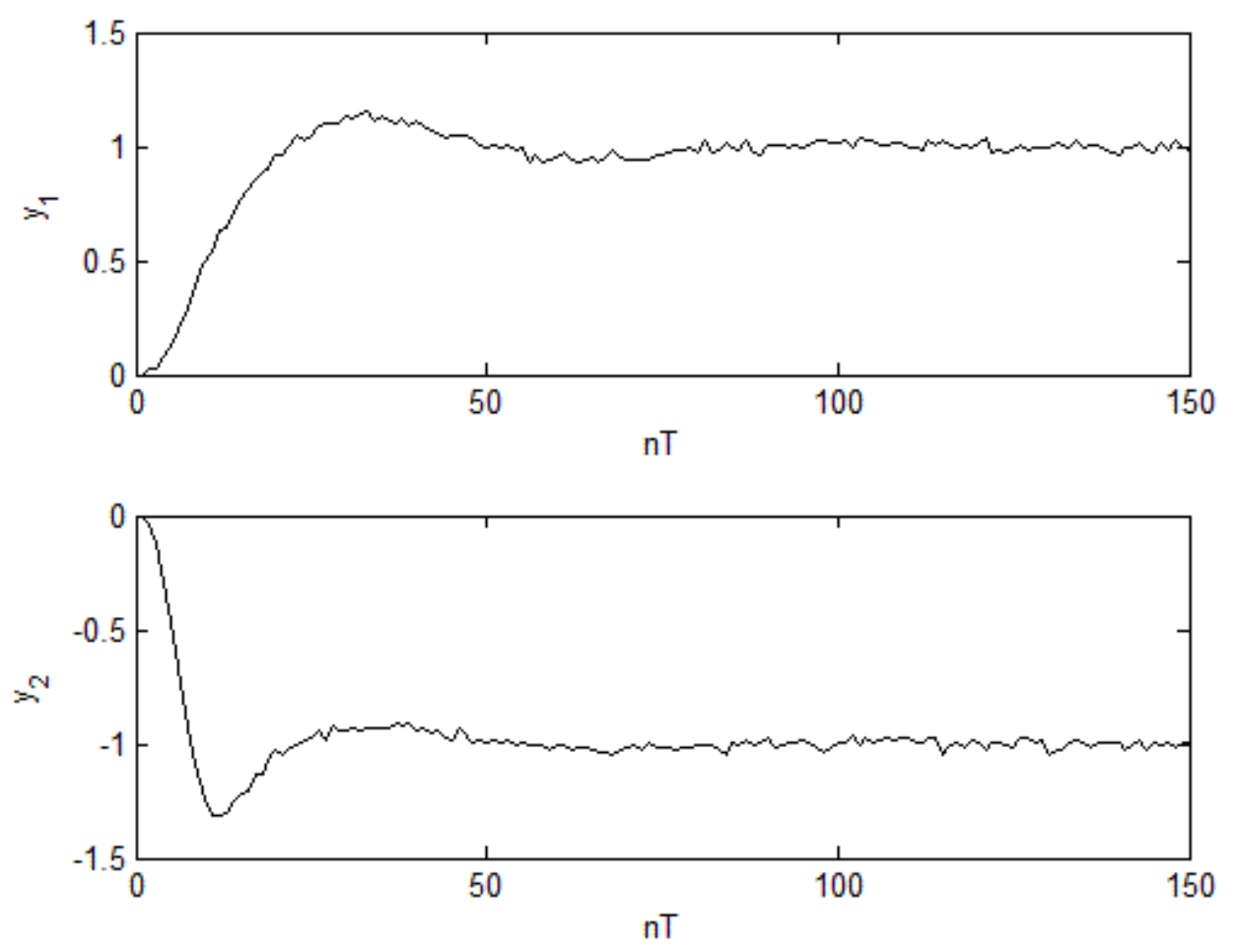

Figura 4.12 - Respostas do controlador MPC sem excitação, com ruído

Para a identificação, seguiu-se o mesmo procedimento das seções anteriores. O conjunto de dados utilizado (1500 pontos) está representado nas figuras 4.13 e 4.14. Como no caso 2, o controlador empregado foi o do Problema (P3), sendo que o 
modelo utilizado pelo MPC para as predições foi o modelo nominal $\boldsymbol{G}_{1}(s)$ enquanto a planta foi representada pelo modelo 3 da tabela 4.1.
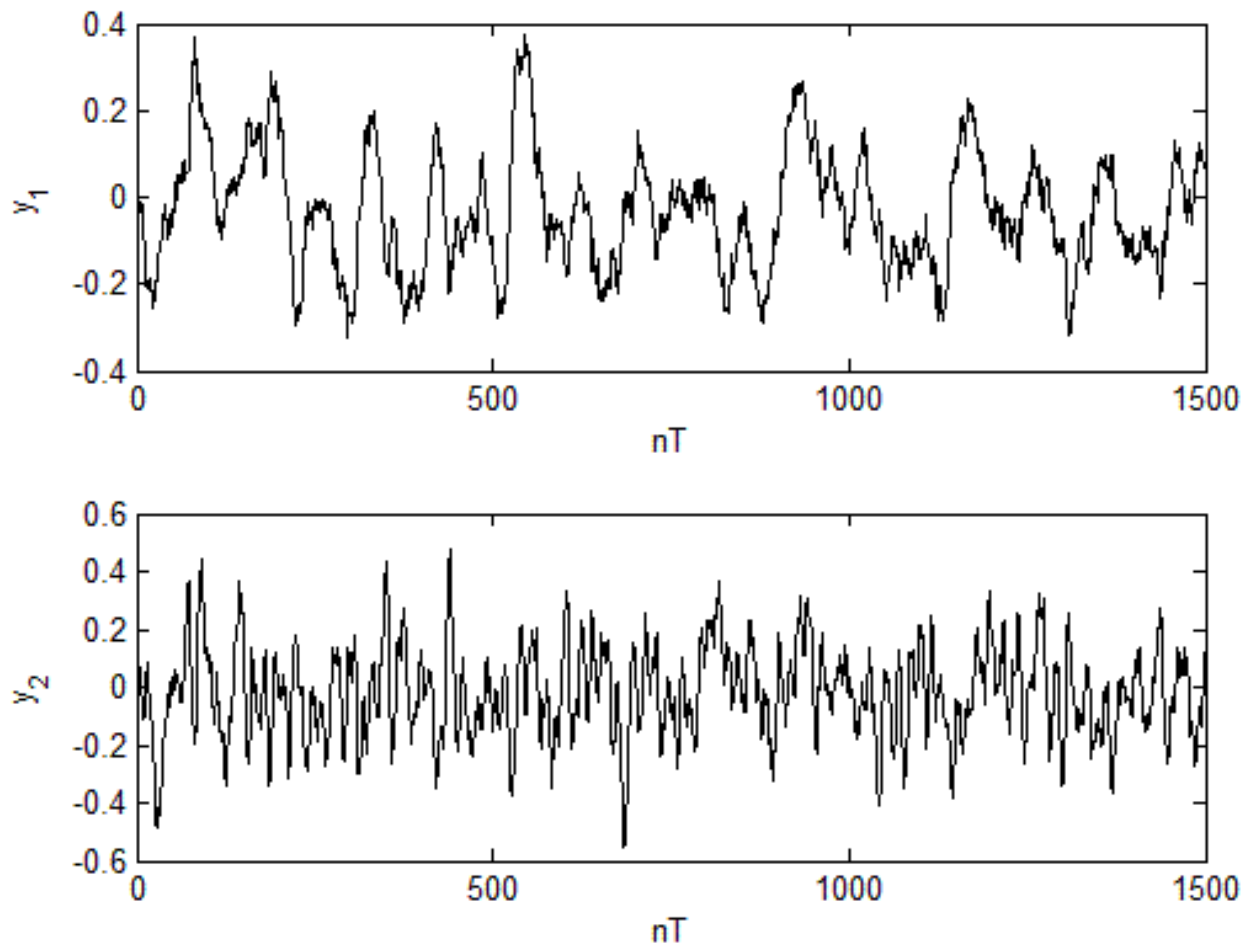

Figura 4.13 - Variáveis controladas da coluna debutanizadora - caso 3
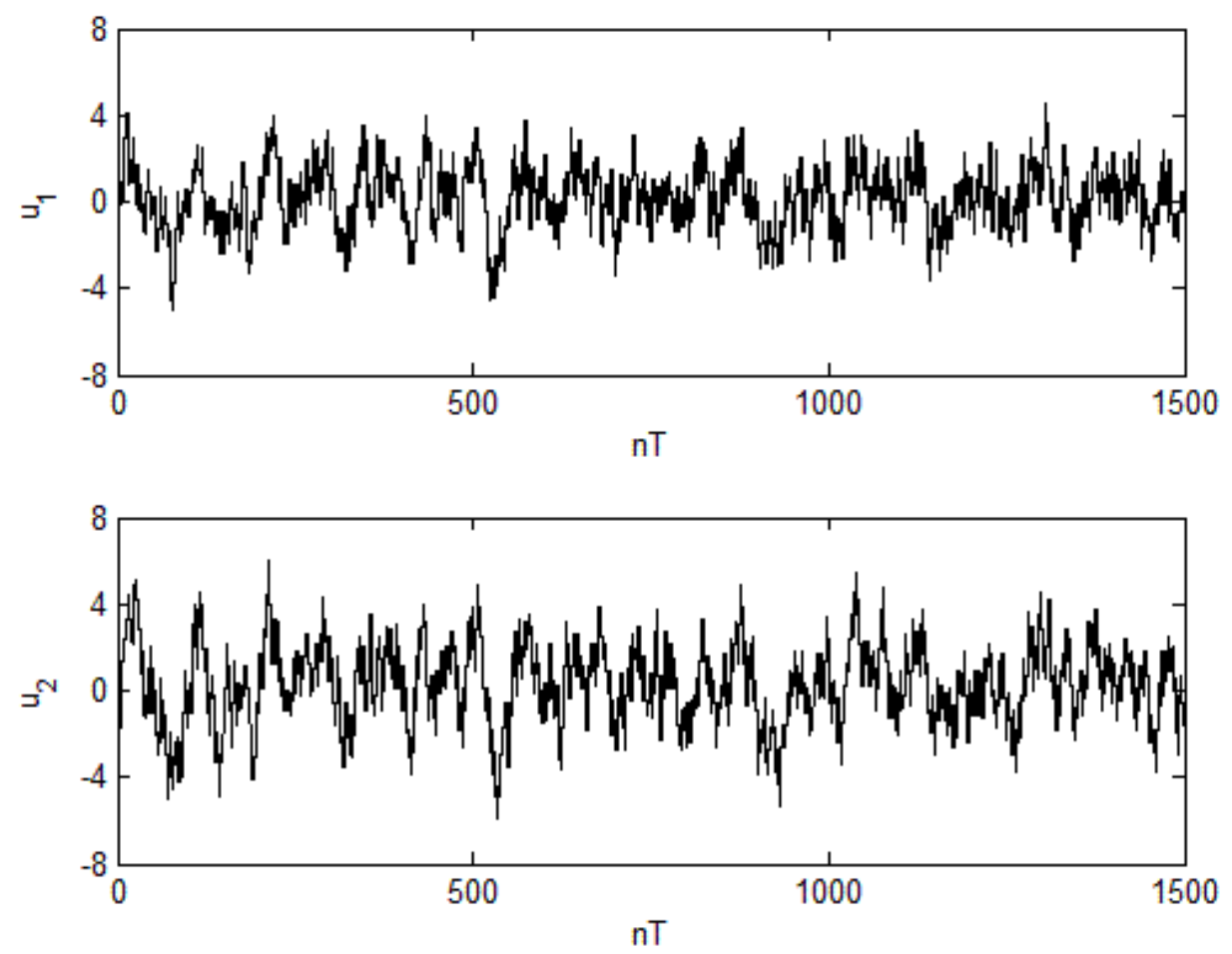

Figura 4.14 - Variáveis manipuladas da coluna debutanizadora - caso 3 
Neste caso, a identificação efetuada na forma dos dois testes anteriores, com os dados sem tratamento e modelo ARX com as mesmas ordens ([na,nb,nk] $=[4,4,1]$ ), não apresentou bons resultados, o que se verificou nos testes de validação cruzada e como se pode constatar nas respostas ao degrau representadas na figura 4.15.
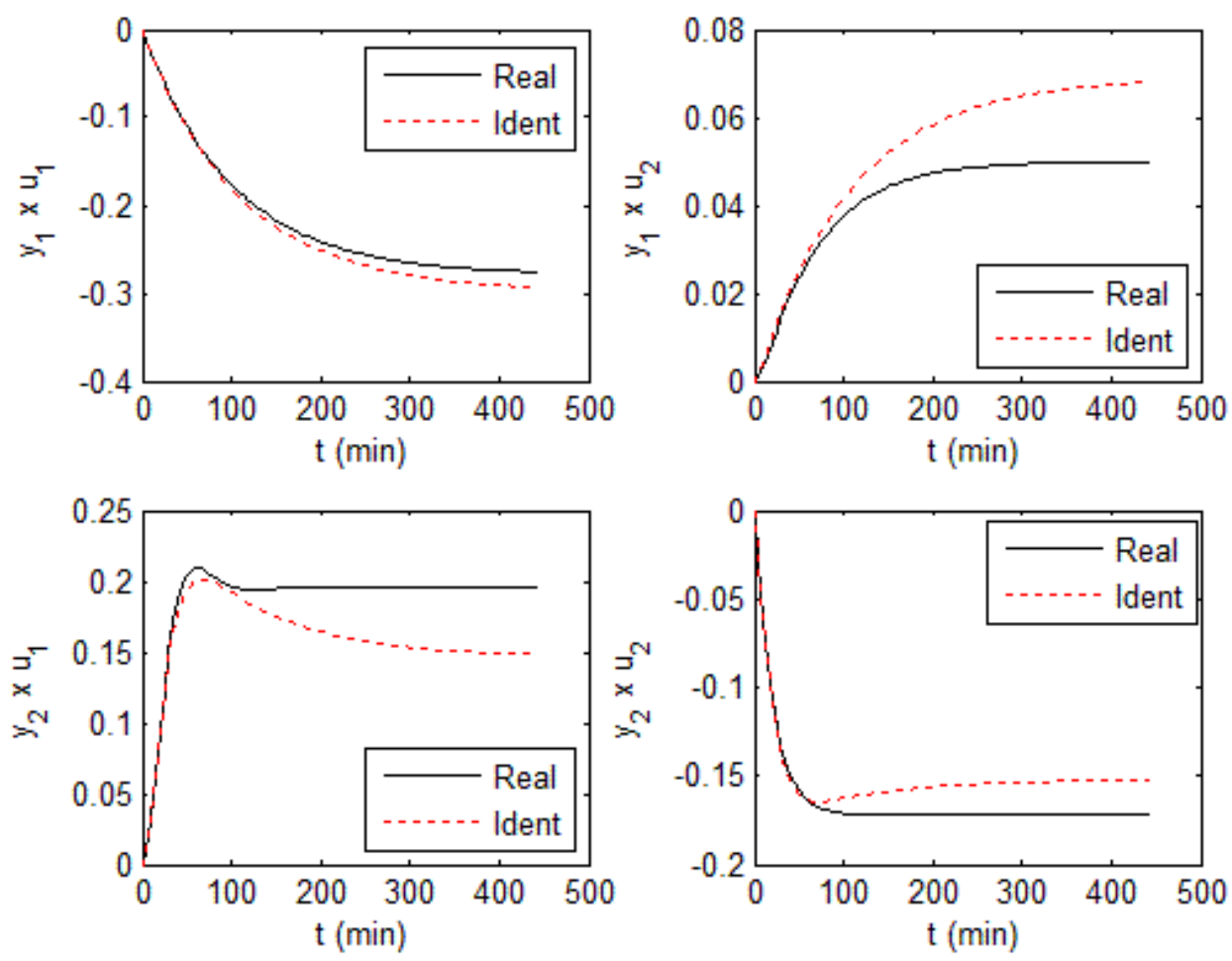

Figura 4.15 - Respostas ao degrau da planta e do modelo identificado - caso 3 (modelo diferente da planta, com ruído - dados sem tratamento)

Após análise do espectro de freqüências dos dados de entrada e saída e sabendose que o processo em estudo é bastante lento, ou seja, que as freqüências de interesse envolvidas são predominantemente baixas, decidiu-se testar a aplicação de um filtro ao conjunto de dados usado na identificação. Foi empregado um filtro passa-baixas com freqüência de corte de $0.05 \mathrm{rad} / \mathrm{min}$.

Os novos resultados da identificação são apresentados nas figuras 4.16 e 4.17, que ilustram respectivamente as avaliações comparativas através da validação cruzada e teste de resposta ao degrau. 

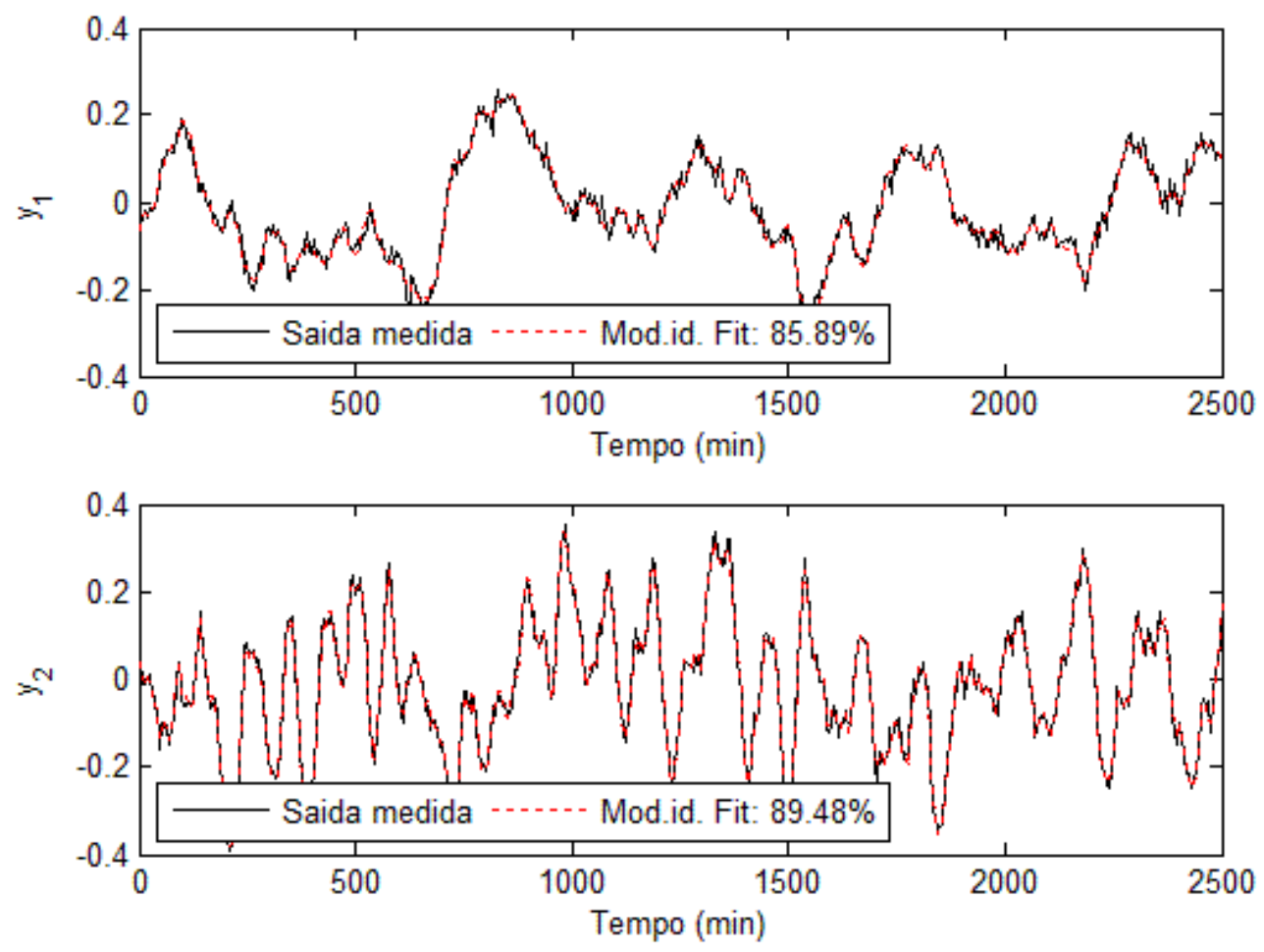

Figura 4.16 - Validação cruzada do modelo identificado - caso 3 (modelo diferente da planta, com ruído - dados filtrados)
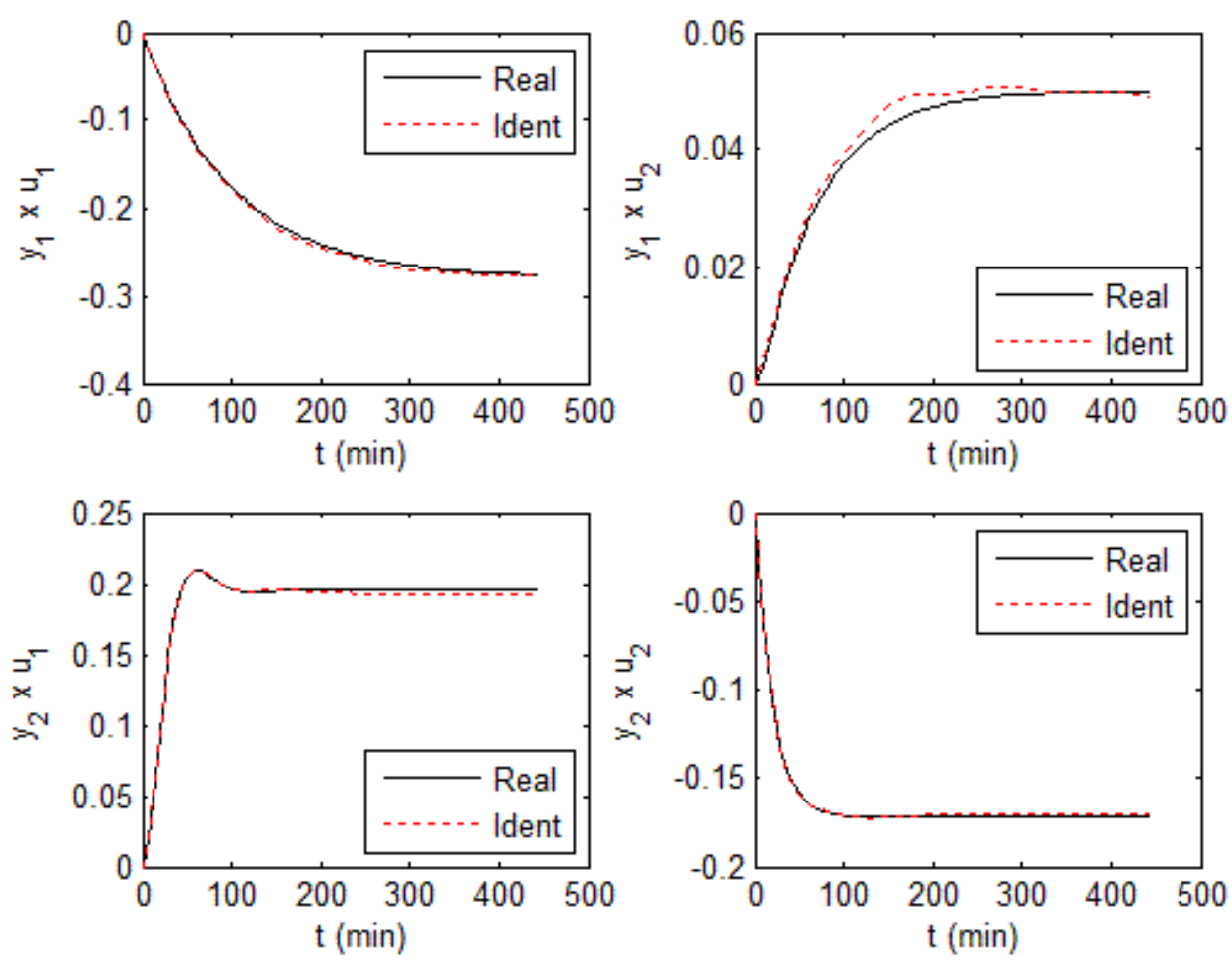

Figura 4.17 - Respostas ao degrau da planta e do modelo identificado - caso 3 (modelo diferente da planta, com ruído - dados filtrados) 
Como se pode observar, com este procedimento os resultados melhoraram bastante em relação ao teste sem filtro nos dados. O teste de validação cruzada (figura 4.16) apresentou um bom ajuste e os testes de resposta ao degrau indicam que o modelo identificado é adequado para o uso a que se propõe, substituindo o modelo anteriormente empregado no MPC.

Este teste também aponta a necessidade de estudos mais aprofundados para o uso do controlador com excitação objeto deste trabalho em casos com ruído, pois mesmo no caso de um ruído pequeno como o utilizado nesta seção já se constatou considerável influência (deterioração) do modelo identificado.

\subsubsection{Teste de identificação - caso 4 - controlador por faixas}

Neste teste empregou-se o controlador MPC por faixas com restrição de excitação Problema (P4) - desenvolvido na seção 3.6 para gerar os dados utilizados na identificação. O procedimento empregado foi o mesmo já descrito na seção 4.1.5 para o caso 2.

O modelo nominal utilizado para as predições do controlador foi o modelo 1 da tabela 4.1 enquanto a planta real foi representada pelo modelo 3 da mesma tabela. A sintonia empregada no controlador foi a mesma apresentada na tabela 3.5 e o erro limite $\left(\boldsymbol{e}_{\lim }\right)$ usado no cálculo do $J_{K \lim }$ foi definido igual à largura da faixa das controladas.

As figuras 4.18 e 4.19 apresentam o comportamento das variáveis controladas e manipuladas neste teste.

A figura 4.20 apresenta o resultado da validação cruzada do modelo obtido, onde se pode comparar as saídas medidas com a saída do modelo identificado. Na mesma figura foi incluída também a resposta do modelo nominal. 

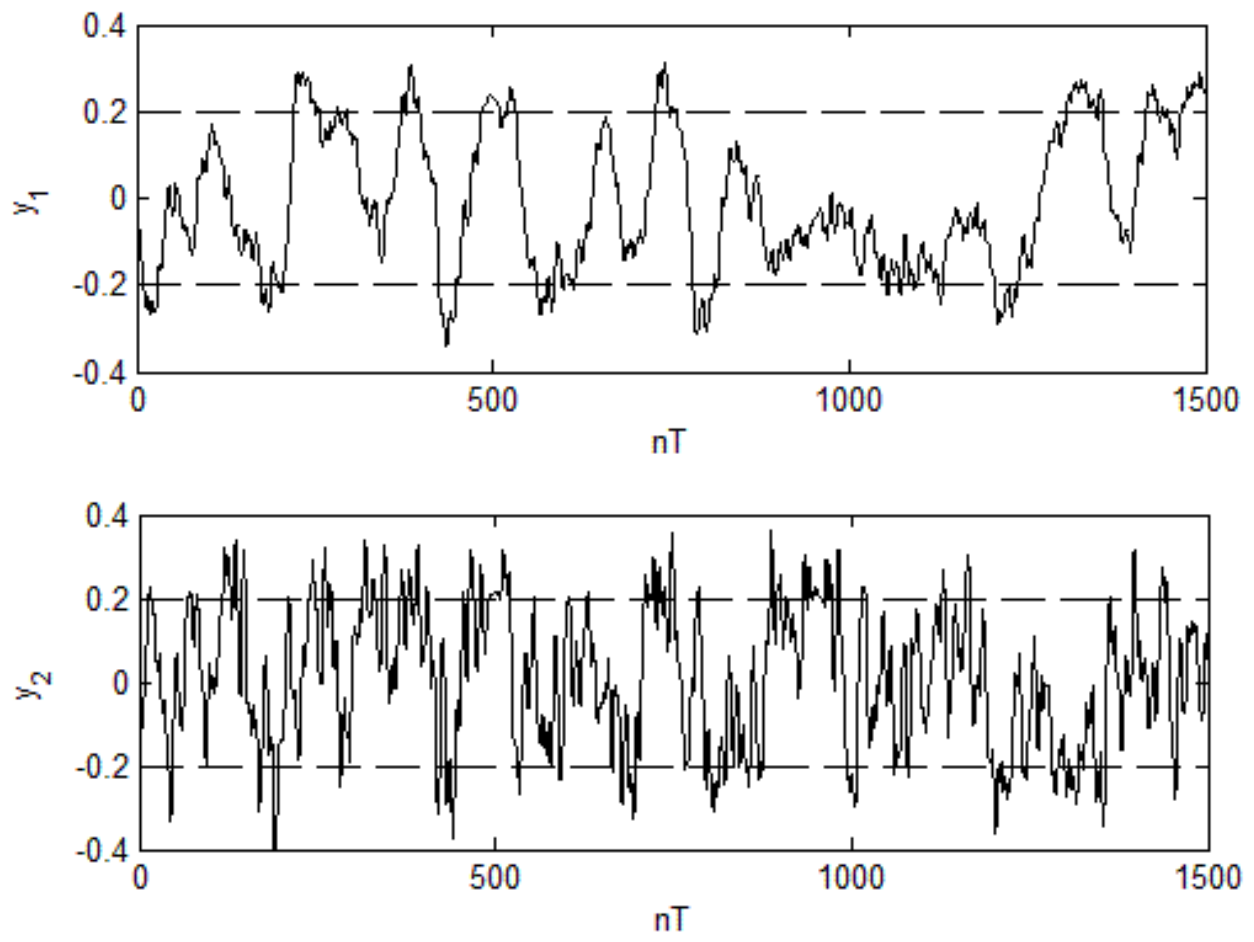

Figura 4.18 - Variáveis controladas da coluna debutanizadora - caso 4
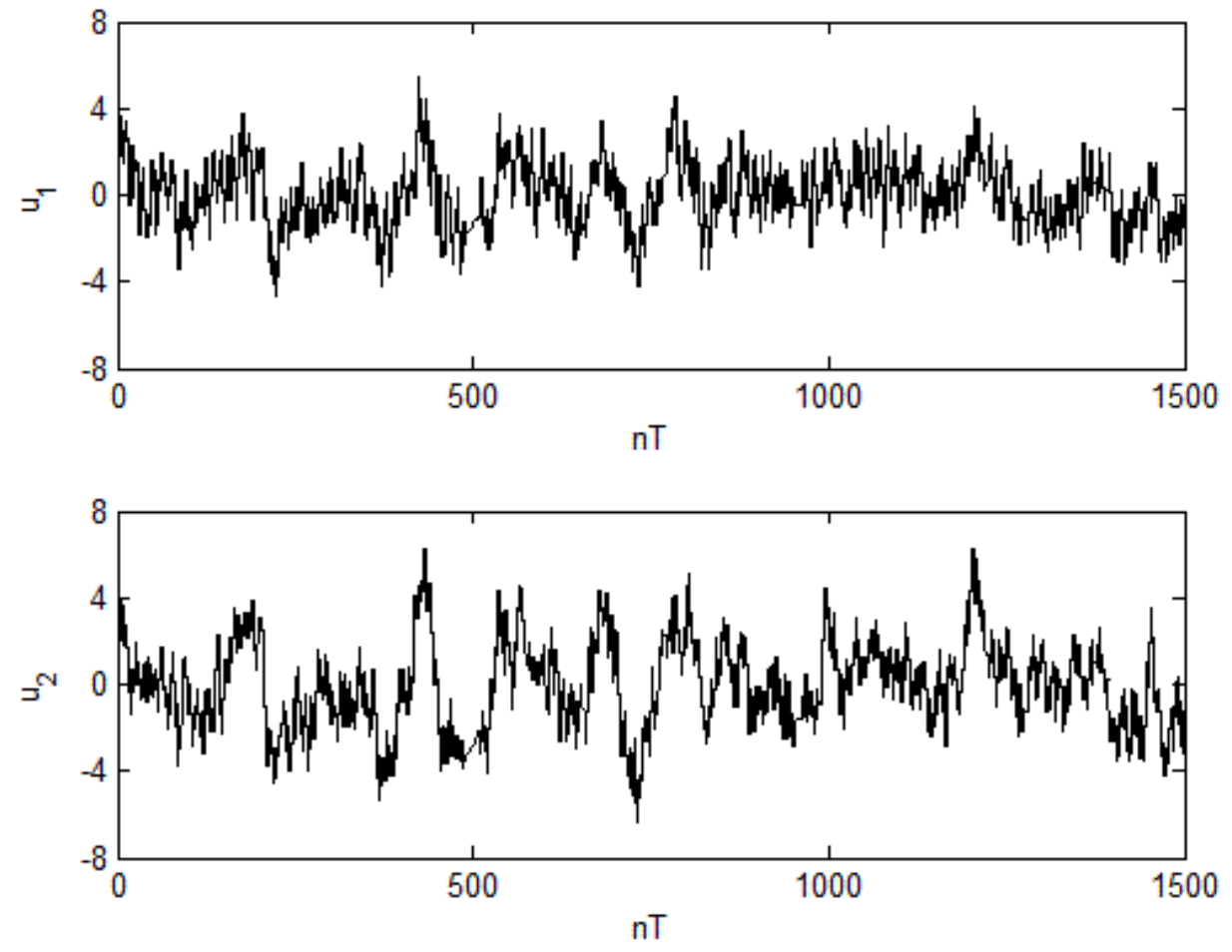

Figura 4.19 - Variáveis manipuladas da coluna debutanizadora - caso 4 

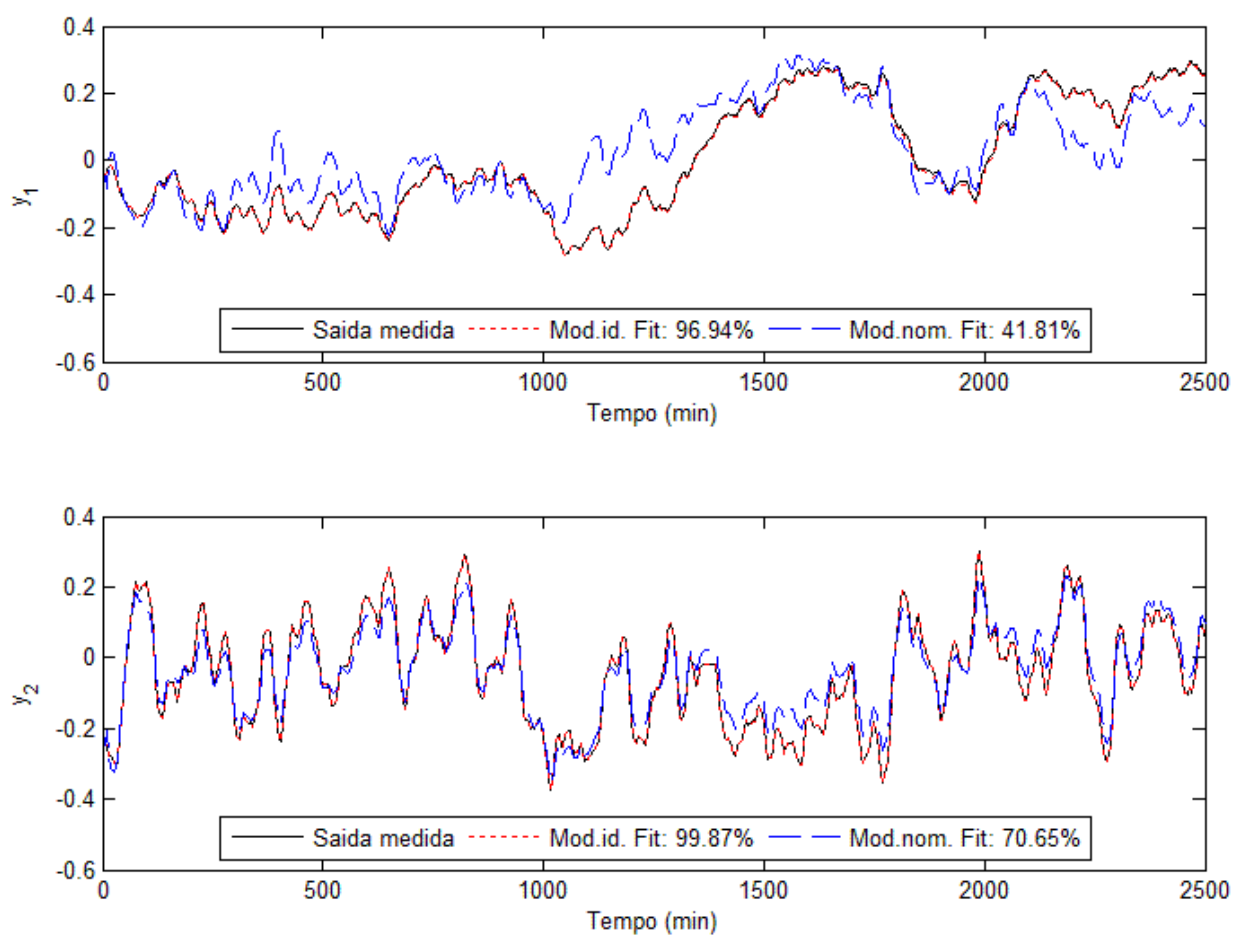

Figura 4.20 - Validação cruzada do modelo identificado - caso 4

A figura 4.21 compara a resposta ao degrau do modelo real, do modelo identificado e do modelo nominal empregado nas predições do MPC.
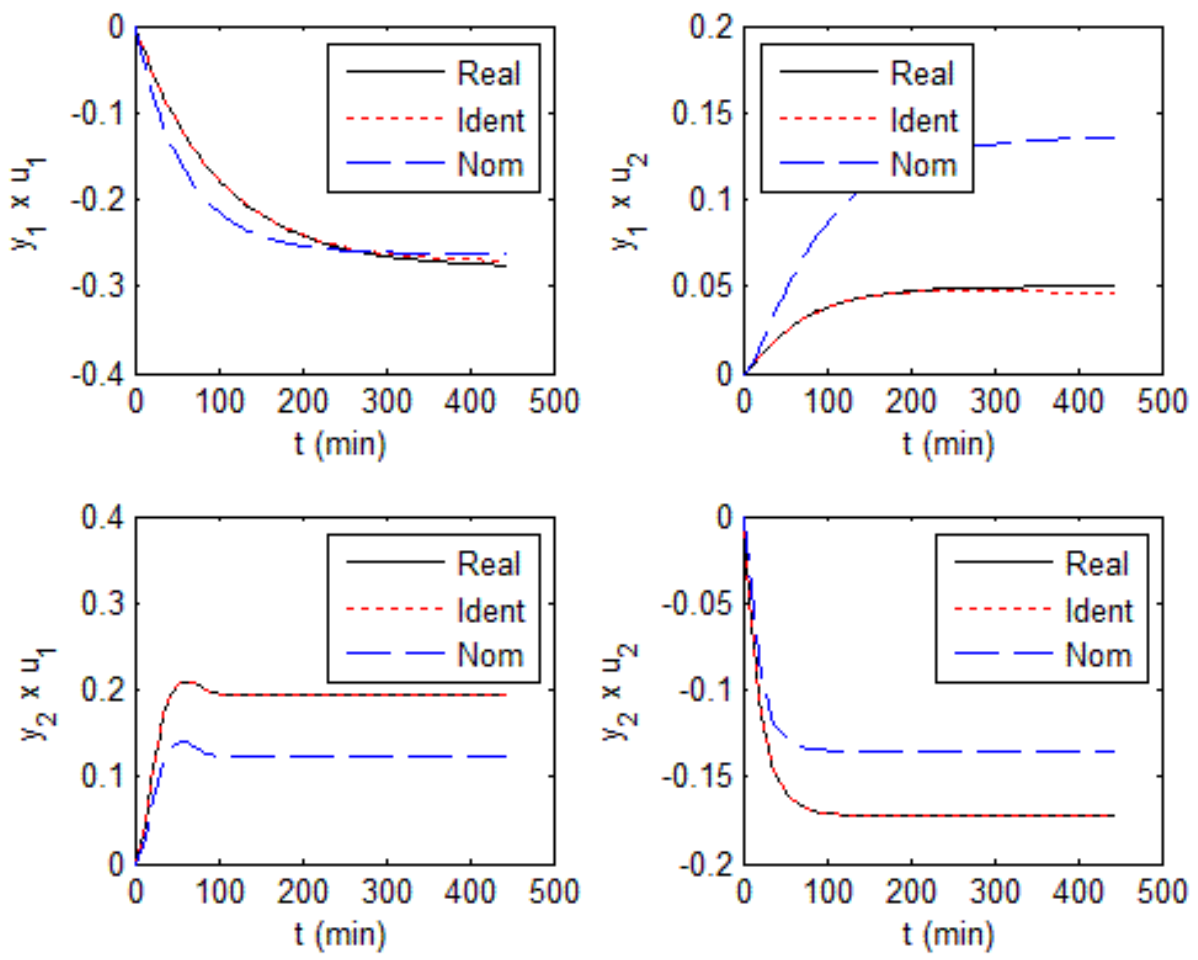

Figura 4.21 - Respostas ao degrau da planta, do modelo identificado e do modelo nominal - caso 4 
Uma primeira observação da figura 4.18 em relação ao caso 2 (figura 4.8) é que neste teste se verificou um maior controle da excitação introduzida no sistema, com as variáveis controladas respeitando mais as faixas definidas.

Quanto à qualidade do modelo obtido, constata-se na figura 4.20 uma pequena degradação no ajuste do modelo para a controlada $y_{1}$, compensada com uma sensível melhoria no ajuste da controlada $y_{2}$. Já na figura 4.21, observa-se que as respostas ao degrau do modelo obtido são aceitáveis para todos os pares controladas $\mathrm{x}$ manipuladas.

Em resumo, a principal vantagem da abordagem $(P 4)$ reside no melhor controle das variáveis. Em termos de qualidade de identificação, os controladores com as formulações $(P 3)$ e $(P 4)$ se mostraram equivalentes.

\subsection{COLUNA C3C4}

\subsubsection{Descrição do processo}

Nesta seção são simulados testes de identificação em um segundo processo sob controle MPC onde os sinais foram gerados mediante a aplicação das abordagens dos Problemas (P3) e (P4) estudadas no capítulo anterior. Trata-se de uma coluna de destilação de uma refinaria da PETROBRAS estudada por Porfírio; Almeida Neto e Odloak (2003), cujo fluxograma de processo simplificado está ilustrado na figura 4.22, onde também é indicada a estratégia do controle MPC bem como algumas outras malhas de controle regulatório.

Neste processo, que é um subsistema de uma unidade de craqueamento catalítico fluído (FCC, do inglês "Fluid Catalytic Cracking"), a corrente C3 (propano e propeno) é separada da corrente C4 (butano, buteno e outros hidrocarbonetos com quatro 
átomos de carbono). A corrente de carga é gás liquefeito de petróleo (GLP) proveniente de uma coluna debutanizadora. Na figura 4.22, T-01 é a coluna de destilação, "E" identifica os trocadores de calor e "V" indica vasos de processo.

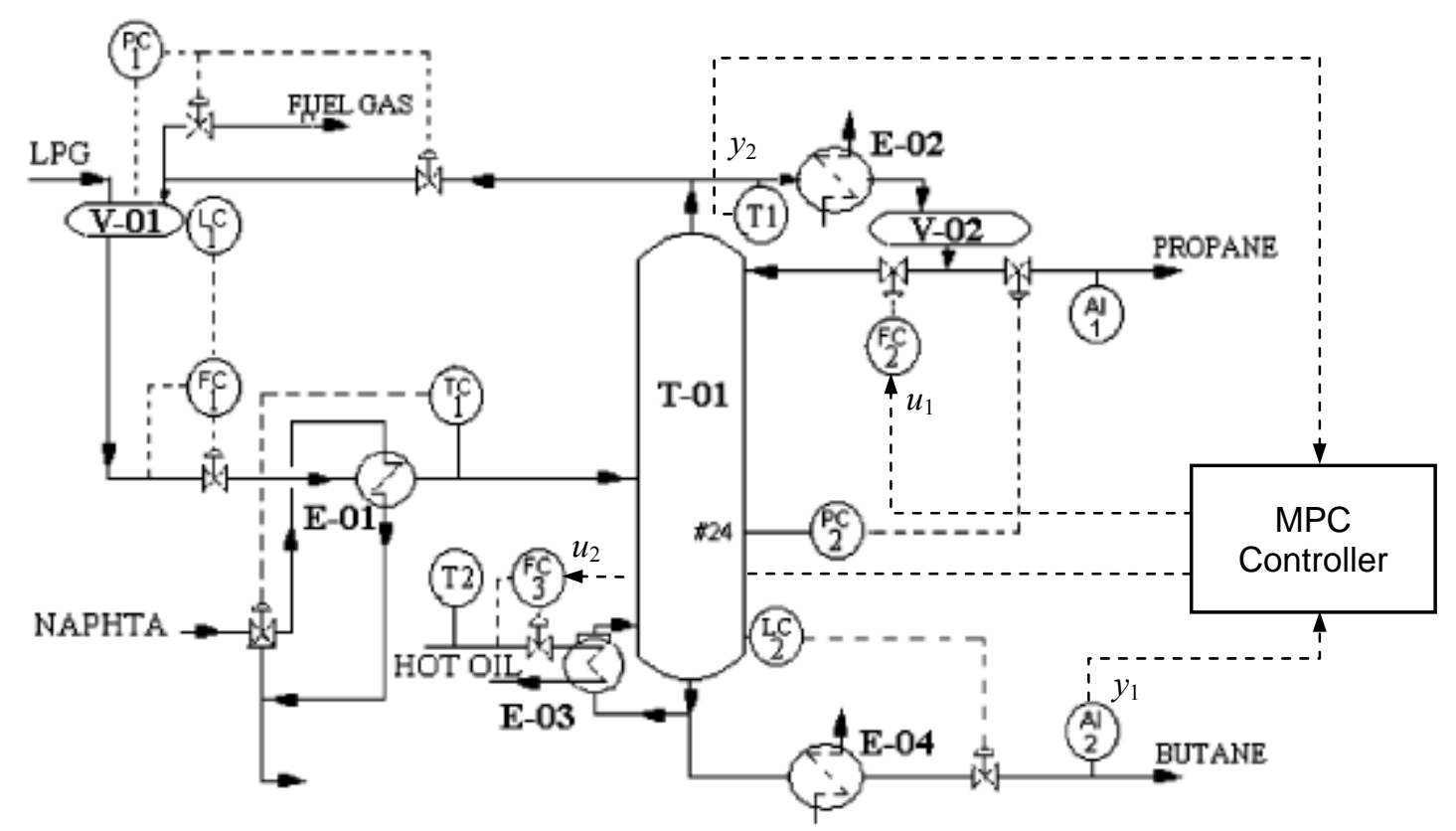

Figura 4.22 - Fluxograma de processo simplificado da coluna C3/C4

A corrente C3 é produzida como a corrente de topo da coluna T-01 e a corrente C4 como a corrente de fundo da coluna. Al1 e Al2 são analizadores que inferem o teor de C4 na corrente de topo e o teor de C3 na corrente de fundo, respectivamente.

O processo deve ser controlado de tal forma que a recuperação de C4 seja maximizada, uma vez que esta corrente é enviada para uma unidade de alquilação onde é convertida em gasolina de alta-octanagem.

Como indicado na figura 4.22, no controlador MPC as variáveis controladas $y_{1}$ e $y_{2}$ são respectivamente a composição molar de C3 na corrente de fundo (AI2 - \%) e a temperatura no primeiro estágio da seção de topo da coluna $\left(\mathrm{T} 1-{ }^{\circ} \mathrm{C}\right)$. As variáveis manipuladas $u_{1}$ e $u_{2}$ são a vazão de refluxo no topo da coluna $\left(\mathrm{FC} 2-\mathrm{m}^{3} / \mathrm{d}\right)$ e a vazão de óleo quente para o refervedor no fundo da coluna (FC3 $-\mathrm{m}^{3} / \mathrm{d}$ ), respectivamente. 
As funções de transferência escolhidas para representar o processo real e o modelo nominal (utilizado no controlador MPC), extraídas do trabalho de Porfírio; Almeida Neto e Odloak (2003), são as indicadas nas eq.(4.7) e (4.8), respectivamente.

$$
\boldsymbol{G}_{p}(s)=\left[\begin{array}{ll}
\frac{\left(-2.218 \times 10^{-4}\right) s+\left(5.656 \times 10^{-4}\right)}{s^{2}+3.4948 s+0.5902} e^{-1 s} & \frac{\left(0.7413 \times 10^{-4}\right) s-\left(1.452 \times 10^{-3}\right)}{s^{2}+2.6987 s+0.4023} e^{-1 s} \\
\frac{\left(-1.135 \times 10^{-3}\right) s-\left(1.235 \times 10^{-3}\right)}{s^{2}+1.6380 s+0.09852} e^{-1 s} & \frac{\left(-0.3 \times 10^{-3}\right) s+\left(0.2 \times 10^{-2}\right)}{s^{2}+2.4298 s+0.0651} e^{-1 s}
\end{array}\right]
$$

$$
\boldsymbol{G}_{m}(s)=\left[\begin{array}{ll}
\frac{\left(0.4227 \times 10^{-4}\right) s+\left(0.1094 \times 10^{-4}\right)}{s^{2}+0.1090 s+0.0243} e^{-1 s} & \frac{\left(-1.2055 \times 10^{-4}\right) s-\left(0.03824 \times 10^{-3}\right)}{s^{2}+0.1342 s+0.0111} e^{-1 s} \\
\frac{\left(-0.0873 \times 10^{-3}\right) s-\left(0.1116 \times 10^{-3}\right)}{s^{2}+0.1317 s+0.0073} e^{-1 s} & \frac{\left(1.3 \times 10^{-3}\right) s+\left(0.7 \times 10^{-2}\right)}{s^{2}+2.2605 s+0.1366} e^{-1 s}
\end{array}\right]
$$

Os principais objetivos dos testes realizados nesta seção foram a aplicação do controlador MPC com restrição de excitação em outro sistema, diferente do empregado na seção 4.1, para fins de avaliação da identificabilidade do processo a partir dos dados gerados e do compromisso com a manutenção do controle das saídas dentro das respectivas faixas. Além disso, permitiram a aplicação das técnicas de identificação de modelos contínuos usadas por Sotomayor; Odloak e Moro (2008) e a comparação dos resultados com os obtidos naquele trabalho para o mesmo processo.

\subsubsection{Caso 5 - Controlador (P3) aplicado à coluna C3/C4}

Neste teste se aplicou o controlador com a formulação do Problema (P3) à coluna C3/C4 descrita em 4.2.1. A sintonia empregada no controlador foi a mesma usada nos artigos de Porfírio; Almeida Neto e Odloak (2003) e Sotomayor; Odloak e Moro (2008), resumida na tabela 4.5, com exceção do horizonte de controle, para o qual se empregou $m=7$ no presente teste contra $m=2$ nos dois trabalhos citados. Isto 
se fez necessário porque como já exposto no capítulo 3, o horizonte de controle do controlador com restrição de excitação objeto deste trabalho deve ser igual ou maior que o rank da pseudo-matriz de informação definida na eq.(3.19) e, nestes testes, se empregou tal matriz com rank=6.

Tabela 4.5 - Sintonia empregada no caso 5 - controlador (P3) aplicado à coluna C3/C4

\begin{tabular}{|c|c|c|c|c|}
\hline \multicolumn{5}{|c|}{ Parâmetros fixos } \\
\hline Horizonte de predição $(n p)$ & 60 & \multirow{3}{*}{$\begin{array}{l}\text { Limites nas } \\
\text { manipuladas }\end{array}$} & $\boldsymbol{u}^{\max }$ & {$\left[\begin{array}{ll}3250 & 2000\end{array}\right]$} \\
\hline Horizonte de controle $(\mathrm{m})$ & 7 & & $u^{\min }$ & {$\left[\begin{array}{ll}2800 & 1500\end{array}\right]$} \\
\hline Set-point controladas $\left(\boldsymbol{y}^{s p}\right)$ & {$\left[\begin{array}{ll}0.75 & 49\end{array}\right]$} & & $\Delta \boldsymbol{u}^{\max }$ & {$\left[\begin{array}{ll}10 & 10\end{array}\right]$} \\
\hline Peso das controladas $(\boldsymbol{Q})$ & {$\left[\begin{array}{ll}1.3 & 1.2\end{array}\right]$} & \multicolumn{2}{|c|}{ Fator de supressão $(\boldsymbol{R})$} & {$\left[\begin{array}{ll}1.5 & 1.5\end{array}\right]$} \\
\hline Tempo de amostragem $\left(T_{s}-\min \right)$ & 1 & & & \\
\hline \multicolumn{5}{|c|}{ Parâmetros de excitação } \\
\hline Fator folga de excitação $1\left(K_{e 1}\right)$ & 300 & \multicolumn{2}{|c|}{ Fator folga de excitação $2\left(K_{e 2}\right)$} & 2 \\
\hline
\end{tabular}

Outro detalhe é que como o controlador do Problema (P3) não trabalha com faixas para as variáveis controladas, neste teste se utilizou como "set-points" do controlador as médias dos limites superior e inferior das faixas de controle das saídas empregadas nos artigos citados. Os parâmetros de excitação indicados na tabela 4.5 foram ajustados por tentativa e erro até se obter um nível de excitação das variáveis julgado adequado.

O procedimento de identificação foi o mesmo usado por Sotomayor; Odloak e Moro (2008), a seguir resumido:

- O teste foi executado por 4500 minutos, resultando num conjunto de dados com 4500 amostras das entradas e saídas.

- O conjunto de dados foi pré-processado, através da remoção das tendências lineares e aplicação de filtro passa-baixas para concentrar a identificação no range de freqüências de interesse.

- Os dados foram divididos em dois subconjuntos: o primeiro com 3000 amostras, usado na identificação propriamente dita, e o segundo com 1500 amostras, reservadas para os testes de validação cruzada dos modelos estimados. 
- Foram executados os procedimentos de identificação, com o uso do "CONTSID toolbox", que trabalha com processos MISO descritos por funções de transferência contínuas (no domínio da freqüência $-s$ ) com denominadores diferentes para cada par entrada/saída.

- A qualidade dos modelos obtidos foi avaliada através da realização de testes de validação cruzada e de resposta ao degrau, em comparação com o modelo nominal empregado pelo MPC e com o modelo teórico (real) do processo.

As figuras 4.23 e 4.24 apresentam, respectivamente, os dados de saída e entrada gerados. Na primeira figura as faixas das variáveis controladas estão indicadas apenas para referência, pois o controlador empregado trabalha com "set-points" fixos. Pode-se observar que embora as variáveis oscilem em torno dos respectivos "set-points", não há como garantir o respeito às faixas de controle desejadas com este controlador, tendo-se verificado ao longo do teste diversos afastamentos, principalmente da variável $y_{2}$, em níveis que poderiam ser inaceitáveis num teste real.
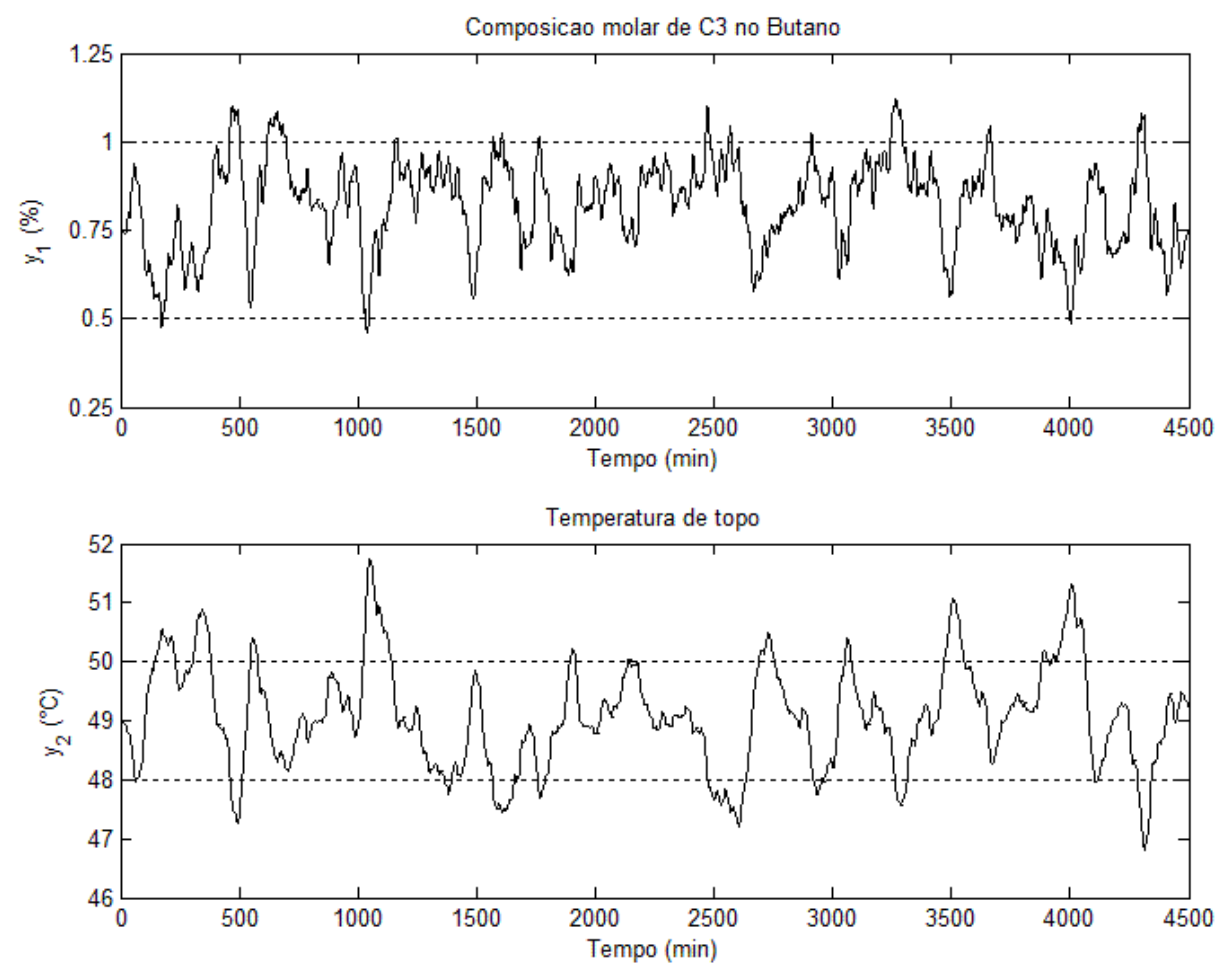

Figura 4.23 - Variáveis controladas da coluna C3/C4 - Caso 5 

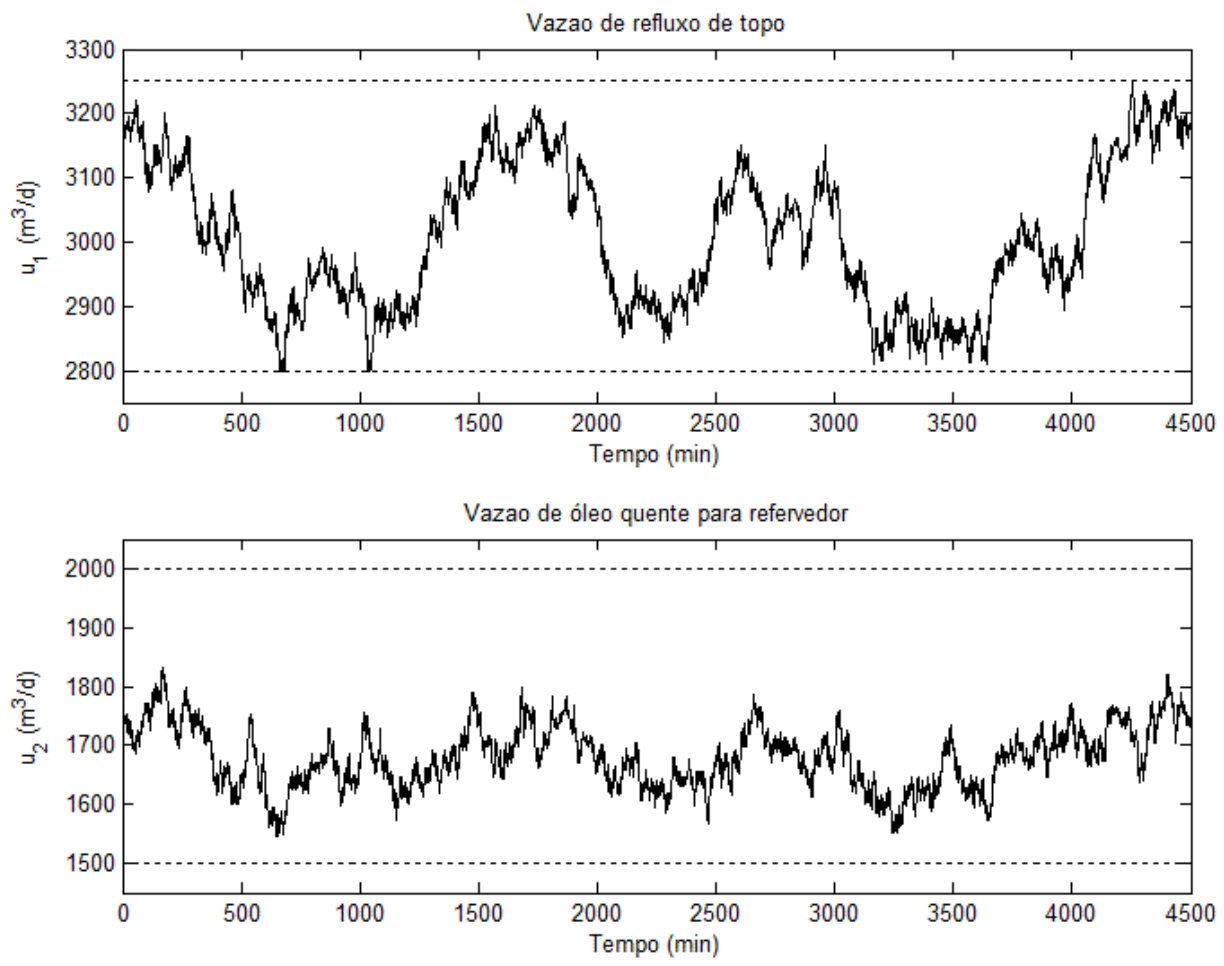

Figura 4.24 - Variáveis manipuladas da coluna C3/C4 - Caso 5

Os resultados dos testes de validação cruzada dos modelos obtidos são apresentados na figura 4.25, juntamente com alguns índices de desempenho. Para comparação, na mesma figura também é apresentada a resposta do modelo nominal usado pelo MPC quando submetido às entradas do conjunto de dados de validação. Pode-se observar que o novo modelo identificado apresenta um ajuste aos dados de validação muito melhor que o modelo nominal, o que sugere que a atualização do controlador para usar o novo modelo no lugar do modelo nominal levaria a um melhor desempenho.

A figura 4.26 compara as respostas ao degrau do modelo teórico (planta real), do modelo nominal e do novo modelo identificado. Observa-se nesta figura que o novo modelo reproduz com uma aproximação muito boa o comportamento do modelo teórico, tanto no que diz respeito ao ganho em estado estacionário quanto em relação às características dinâmicas do sistema, o que não se verifica nas respostas do modelo nominal. Novamente, pode-se afirmar que as predições do controlador MPC seriam muito melhores se fosse usado o novo modelo no lugar do modelo nominal. 

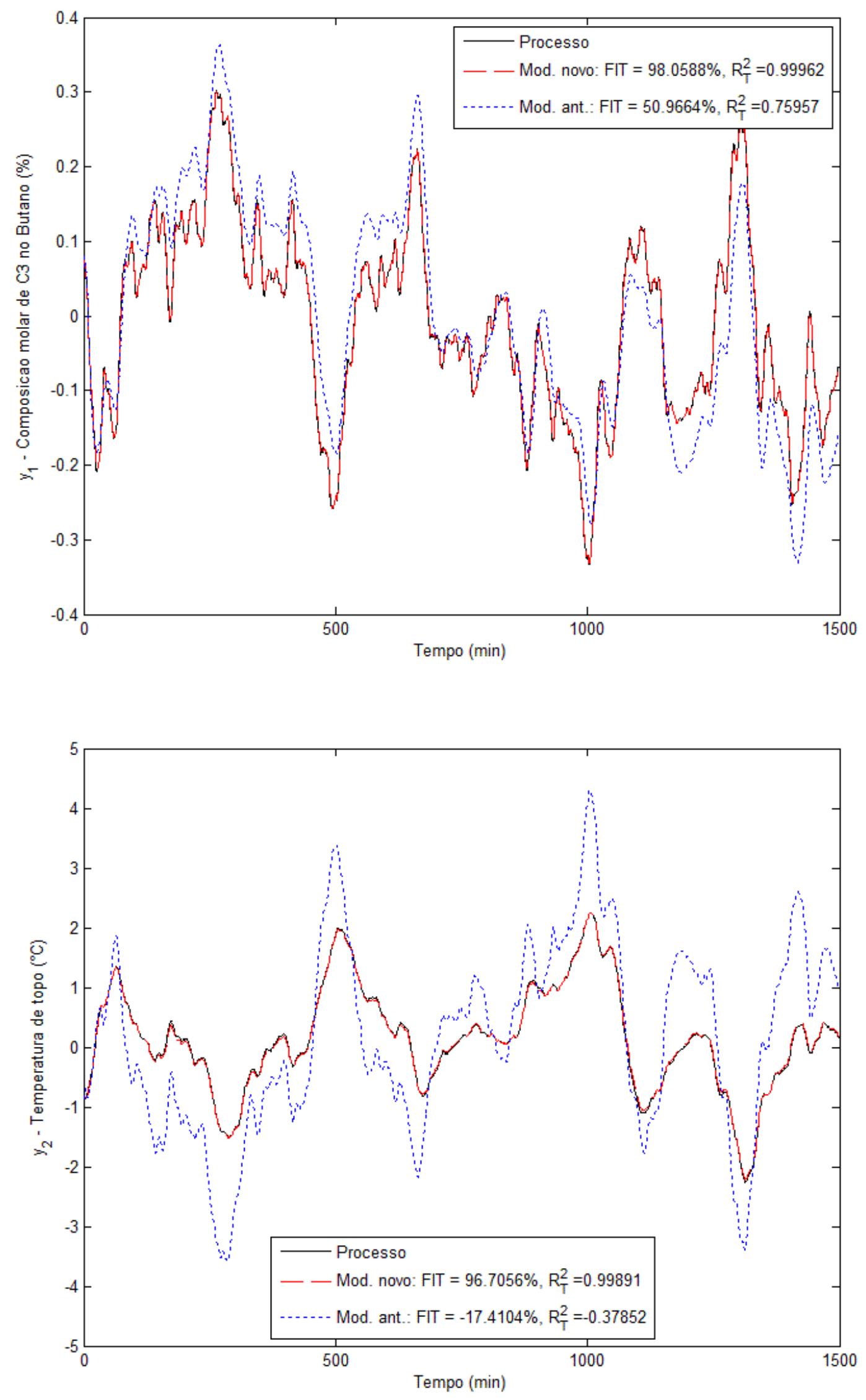

Figura 4.25 - Validação cruzada dos modelos identificados - Caso 5 

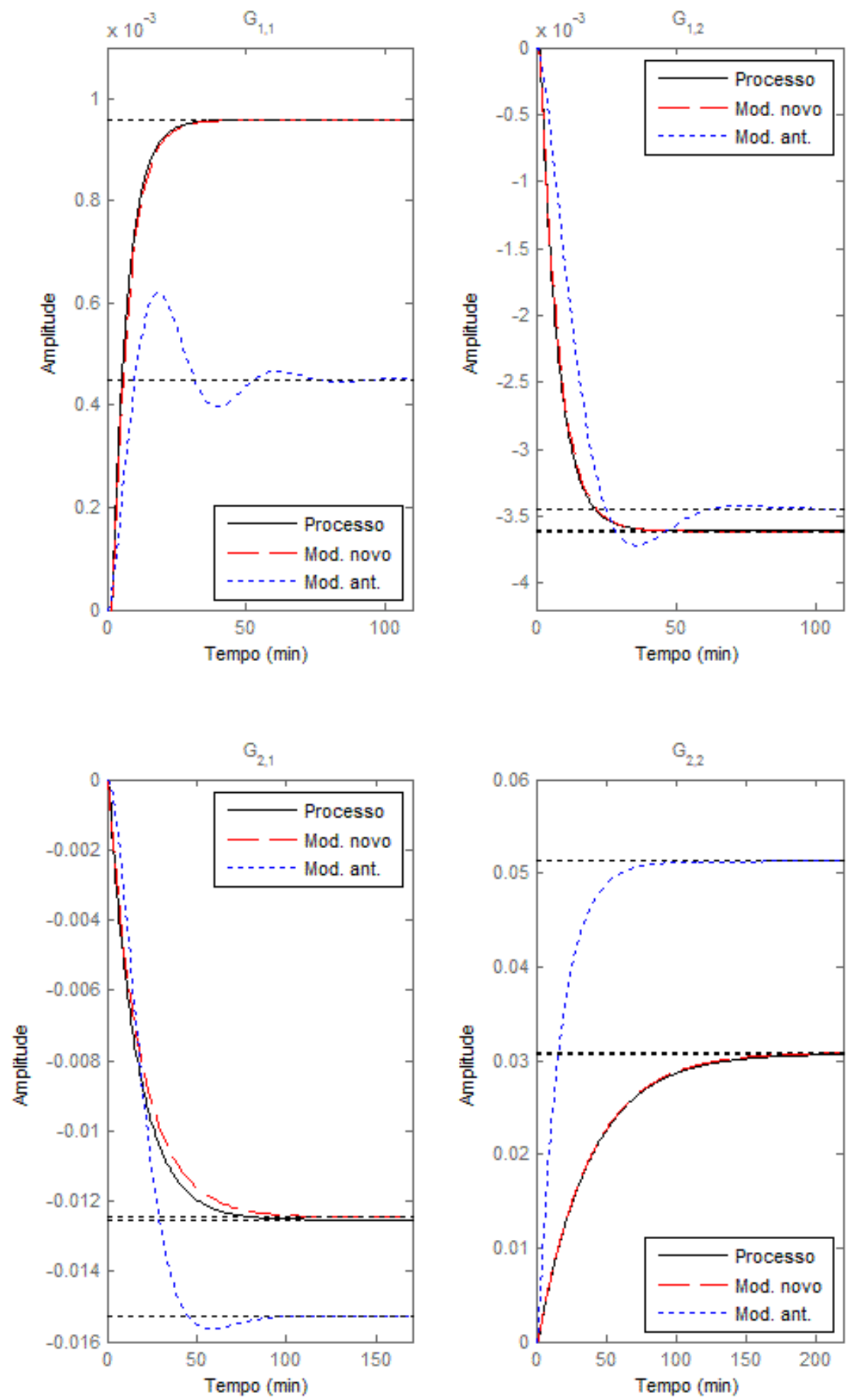

Figura 4.26 - Respostas ao degrau dos modelos da coluna C3/C4 - Caso 5

Os índices de ajuste obtidos nos testes de validação cruzada e apresentados na figura 4.25 foram similares aos obtidos no trabalho de Sotomayor; Odloak e Moro (2008). A tabela 4.6 compara os valores dos índices obtidos naquele trabalho e nos 
testes desta seção. Entretanto, ao se analisar esta tabela, deve ser feita a ressalva de que são índices calculados sobre conjuntos de dados diferentes obtidos em testes diferentes e, portanto, as pequenas diferenças observadas não nos permitem afirmar que os modelos obtidos por um método sejam melhores ou piores que os obtidos pelo outro.

Tabela 4.6 - Comparação dos índices de ajuste obtidos no teste de validação cruzada para a Coluna C3/C4

\begin{tabular}{|c|c|c|c|}
\hline & & \\
\hline & & FIT (\%) & $R_{T}^{2}$ \\
\hline \multirow{2}{*}{$\begin{array}{l}\text { Sotomayor; Odloak } \\
\text { e Moro (2008) }\end{array}$} & $y_{1}$ & 98,0728 & 0,99963 \\
\hline & $y_{2}$ & 96,6428 & 0,99887 \\
\hline \multirow{2}{*}{ Caso 5 - controlador $(P 3)$} & $y_{1}$ & 98,0588 & 0,99962 \\
\hline & $y_{2}$ & 96,7056 & 0,99891 \\
\hline
\end{tabular}

Na figura 4.27 são apresentadas as respostas ao degrau obtidas no trabalho de Sotomayor; Odloak e Moro (2008), para comparação com os resultados obtidos nos testes da presente seção.

De modo geral, os resultados da identificação produzidos neste teste foram bastante satisfatórios. Em comparação com os resultados apresentados no trabalho de Sotomayor; Odloak e Moro (2008), observa-se que os índices de ajuste verificados no teste de validação cruzada resumidos na tabela 4.6 foram muito similares. $O$ mesmo se observa nos gráficos de respostas ao degrau das figuras 4.26 e 4.27.

É importante lembrar que o controlador do Problema (P3) empregado nos testes desta seção trabalha com "set-points" fixos, enquanto o controlador usado no citado artigo trabalha com faixas de controle para as saídas, num algoritmo tal que enquanto as saídas se encontram dentro das respectivas faixas elas não são consideradas na função-objetivo do controlador. Com isto, o teste de identificação executado naquele trabalho é muito próximo de uma situação de teste em malha aberta. 

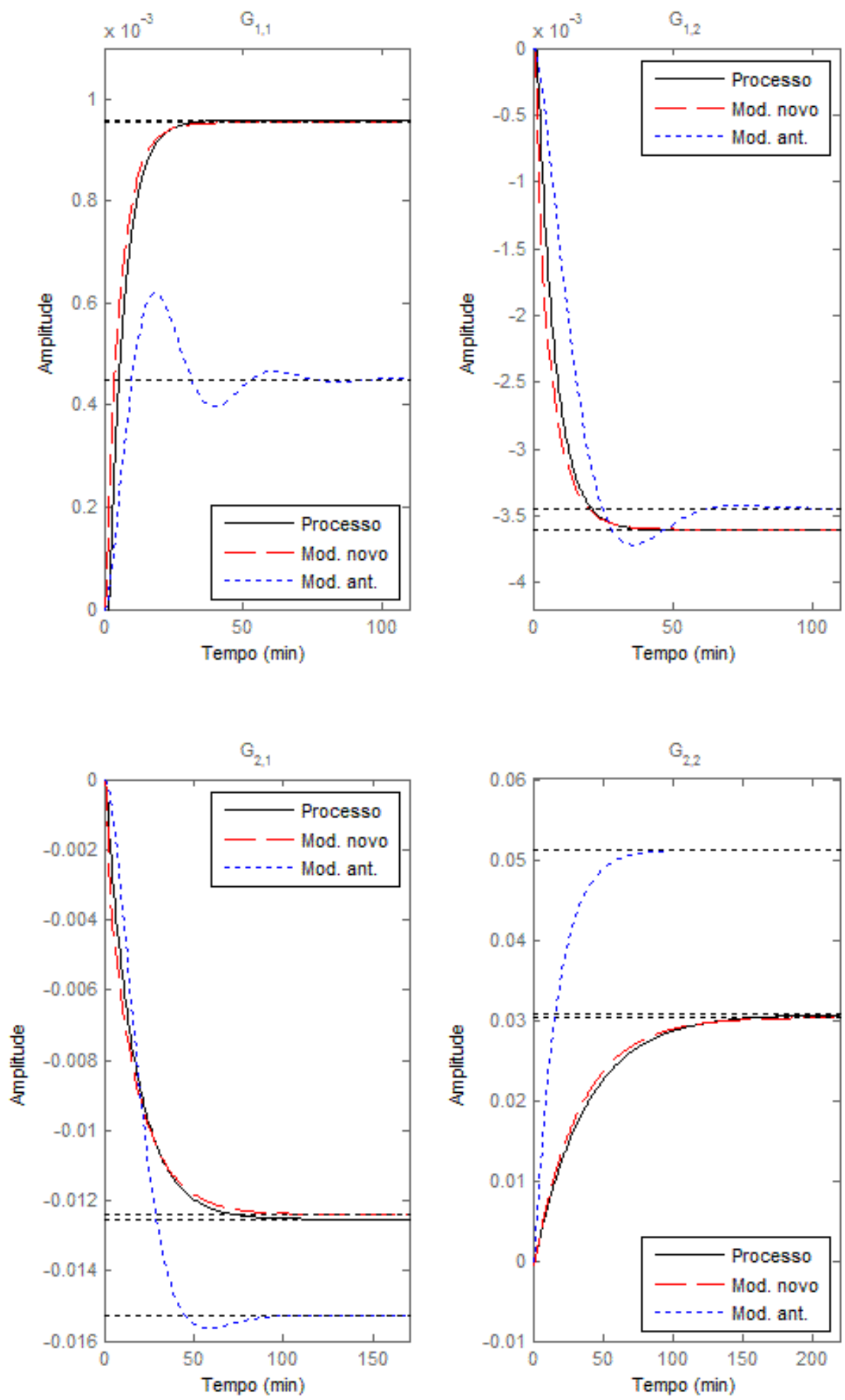

Figura 4.27 - Respostas ao degrau dos modelos da coluna C3/C4 obtidas nos trabalhos de Sotomayor; Odloak e Moro (2008) 


\subsubsection{Caso 6 - Controlador (P4) aplicado à coluna C3/C4}

Nesta seção são descritos os resultados do teste de identificação utilizando o controlador com a formulação do Problema (P4) aplicado à coluna C3/C4 descrita na seção 4.2.1. Os procedimentos de identificação foram os mesmos já detalhados na seção anterior. A sintonia empregada no controlador foi a mesma apresentada na tabela 4.5, com a diferença que neste caso não são definidos valores fixos para os "set-points", mas os seguintes limites para as variáveis controladas: $y^{\max }=\left[\begin{array}{ll}1 & 50\end{array}\right] \mathrm{e}$ $\boldsymbol{y}^{\min }=\left[\begin{array}{ll}0.5 & 48\end{array}\right]$. O erro limite $\left(\boldsymbol{e}_{\lim }\right)$ usado no cálculo do $J_{K \lim }$ para fins de controle da excitação máxima foi definido igual à metade da largura da faixa das controladas.

As figuras 4.28 e 4.29 ilustram o comportamento das variáveis controladas e manipuladas durante o teste, onde se pode perceber um melhor respeito às faixas definidas em relação ao caso da seção anterior.
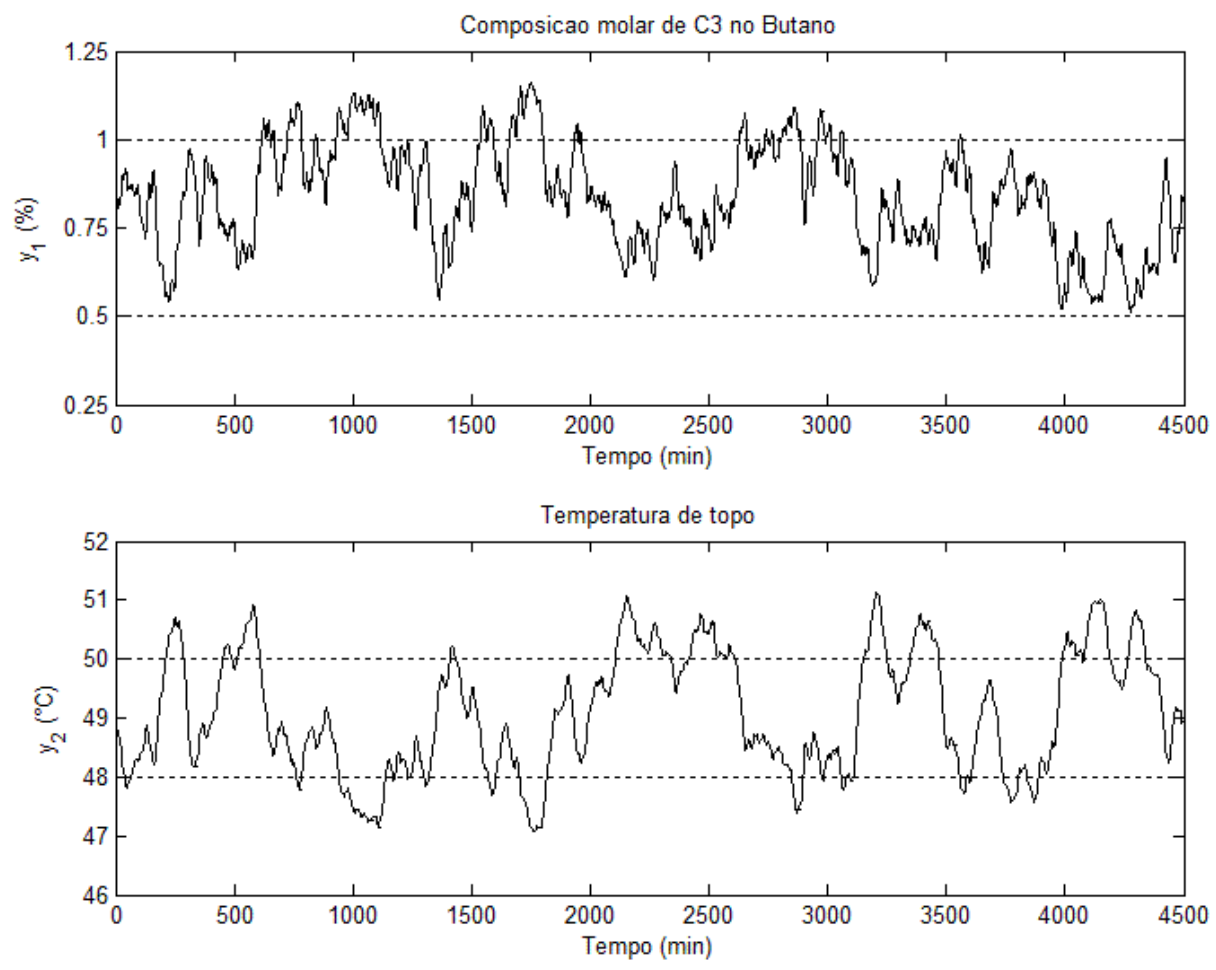

Figura 4.28 - Variáveis controladas da coluna C3/C4 - Caso 6 

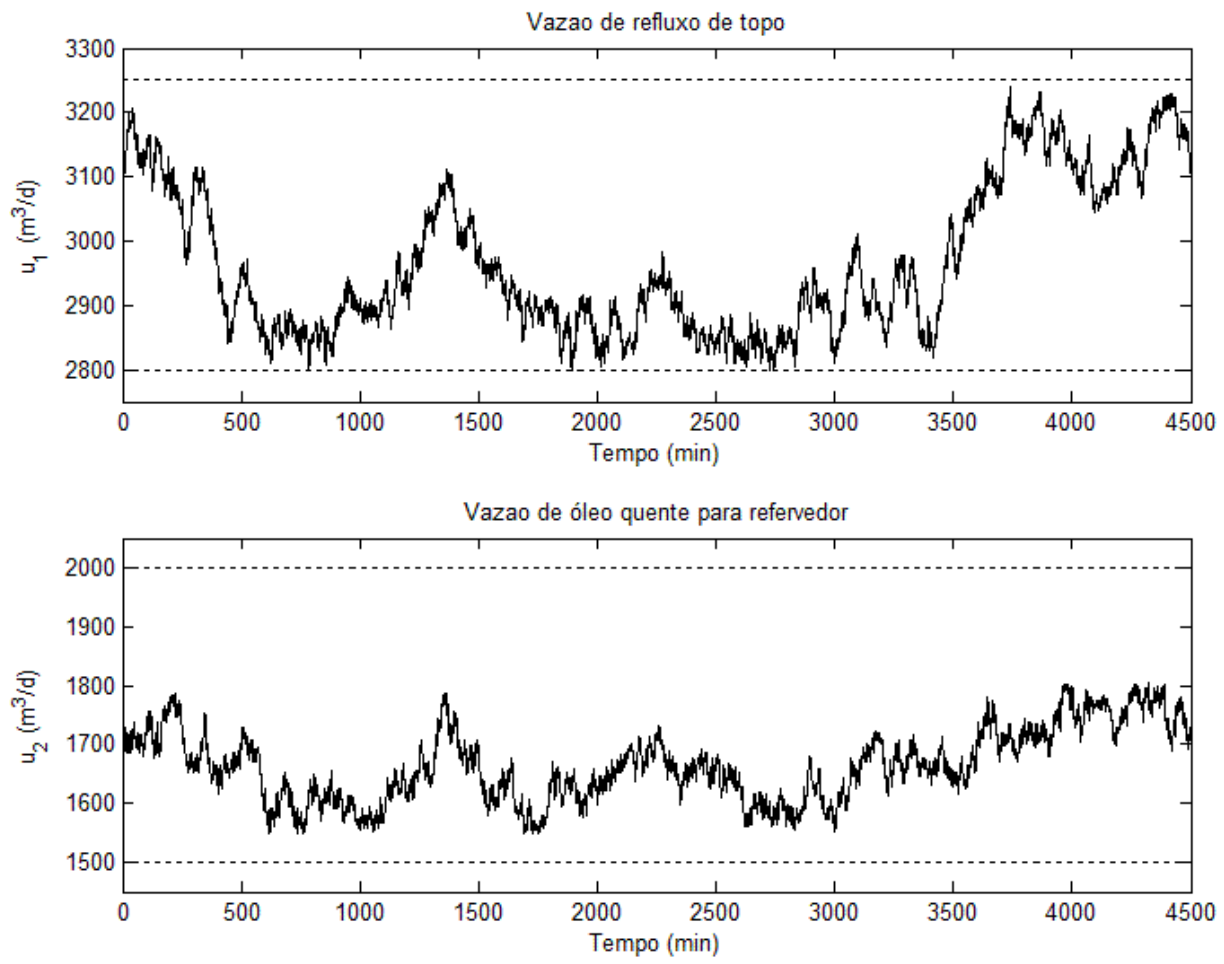

Figura 4.29 - Variáveis manipuladas da coluna C3/C4 - Caso 6

A figura 4.30 apresenta os resultados dos testes de validação cruzada do modelo identificado em comparação com a resposta do modelo antigo (nominal), enquanto as respostas ao degrau do modelo teórico da planta, do modelo identificado e do modelo nominal são comparadas na figura 4.31 .

Pode-se observar na figura 4.30 uma boa aderência da saída do modelo identificado aos dados de validação, com um comportamento muito melhor que o do modelo antigo.

O teste de resposta ao degrau ilustrado na figura 4.31 indica que o modelo identificado reproduziu as características do modelo teórico de uma forma bem melhor que o modelo nominal em uso pelo MPC. A comparação com os resultados do teste da seção anterior com o controlador usando a formulação do Problema (P3) nos mostra que os ajustes foram muito parecidos, com uma pequena degradação na identificação do par $y_{2} \times u_{1}$ no presente teste. 

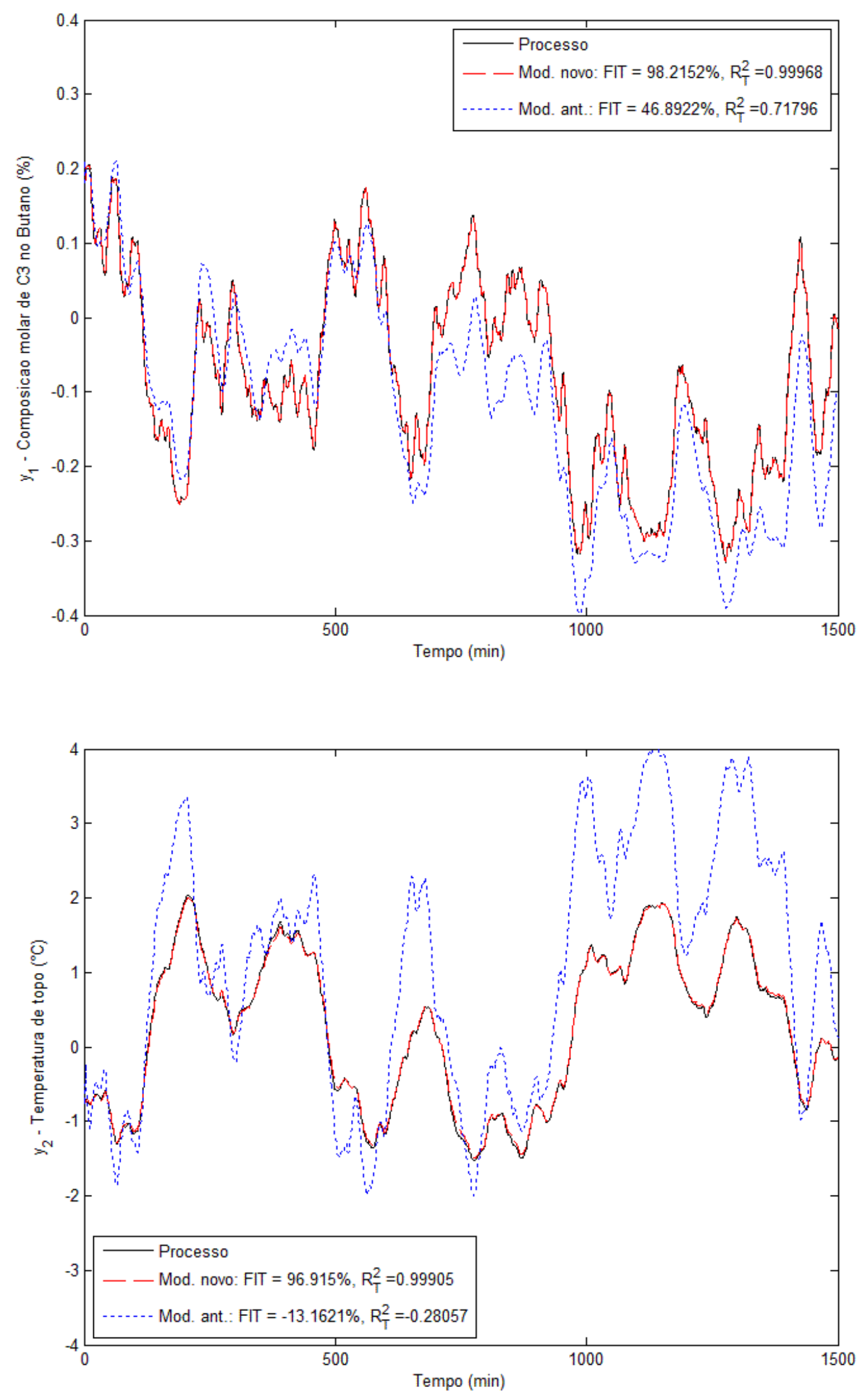

Figura 4.30 - Validação cruzada dos modelos identificados - Caso 6 

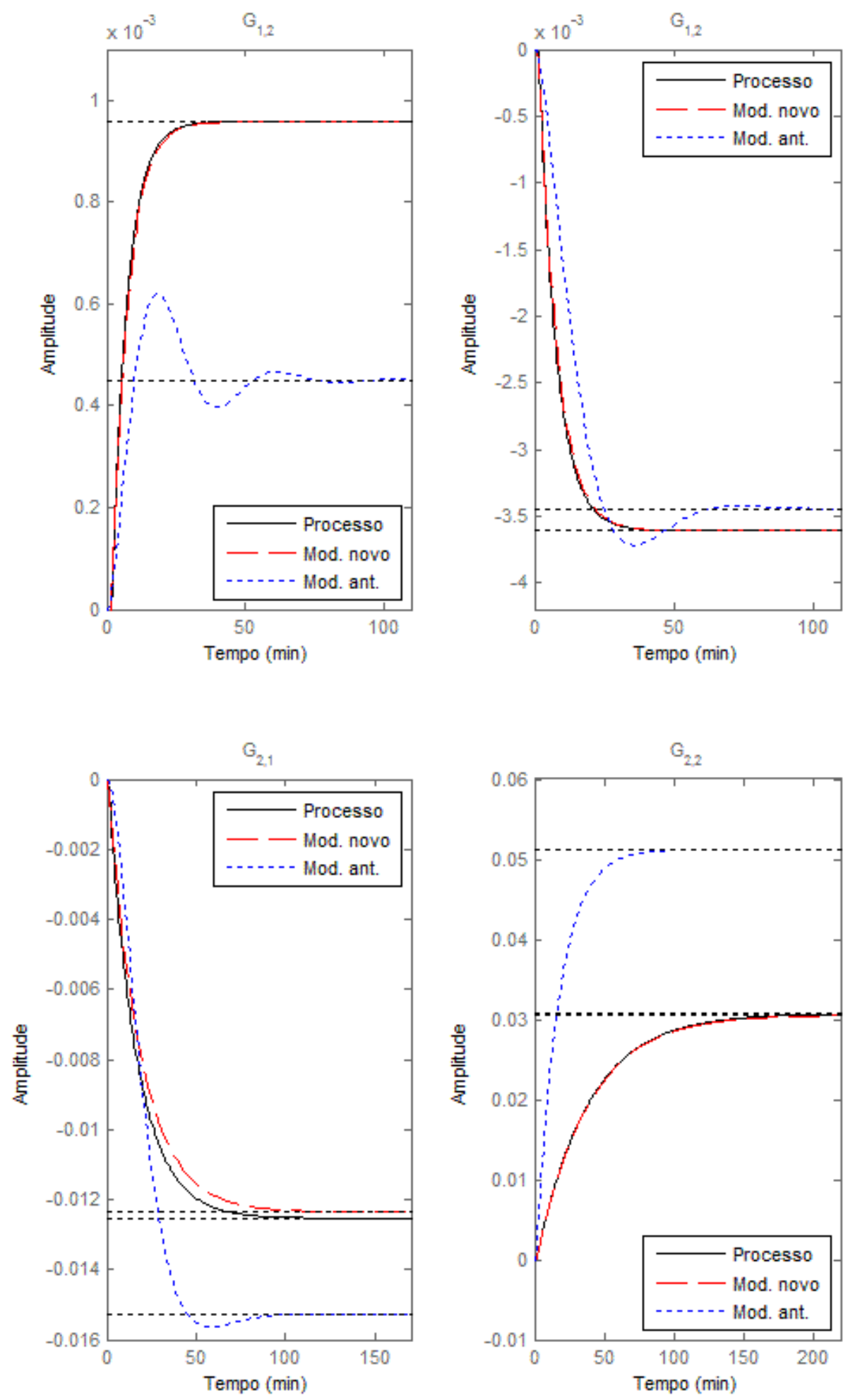

Figura 4.31 - Respostas ao degrau dos modelos da coluna C3/C4 - Caso 6

Outra característica que se pode observar neste teste em relação ao realizado com o uso do controlador com a formulação (P3) é a menor variância dos dados, resultado 
do compromisso de respeito às faixas definidas para as variáveis controladas. Este compromisso entre a variação dos sinais desejável para fins de identificação e o respeito aos requisitos de controle do sistema em teste será uma preocupação sempre presente em qualquer procedimento de identificação em malha fechada.

Novamente, a comparação com os resultados apresentados no trabalho de Sotomayor; Odloak e Moro (2008) mostra resultados muito similares como se pode observar confrontando as figuras 4.31 e 4.27 e os índices de desempenho obtidos nos testes de validação cruzada constantes na tabela 4.7 .

Tabela 4.7 - Comparação dos índices de ajuste obtidos no teste de validação cruzada para a Coluna C3/C4

\begin{tabular}{|l|l|l|l|}
\cline { 3 - 4 } \multicolumn{2}{c|}{} & FIT (\%) & $R_{T}{ }^{2}$ \\
\hline \multirow{2}{*}{$\begin{array}{l}\text { Sotomayor; Odloak } \\
\text { e Moro (2008) }\end{array}$} & $y_{1}$ & 98,0728 & 0,99963 \\
\cline { 2 - 4 } & $y_{2}$ & 96,6428 & 0,99887 \\
\hline \multirow{2}{*}{ Caso 5 - controlador (P3) } & $y_{1}$ & 98,0588 & 0,99962 \\
\cline { 2 - 4 } & $y_{2}$ & 96,7056 & 0,99891 \\
\hline \multirow{2}{*}{ Caso 6 - controlador (P4) } & $y_{1}$ & 98,2152 & 0,99968 \\
\cline { 2 - 4 } & $y_{2}$ & 96,9150 & 0,99905 \\
\hline
\end{tabular}

Embora não tenha prejudicado o procedimento de identificação no presente teste, uma desvantagem do controlador do Problema (P4) em relação ao empregado no trabalho de Sotomayor; Odloak e Moro (2008) é a maior probabilidade de correlação entre as variáveis, pois, como já comentado na seção anterior, o controlador do citado trabalho utiliza um algoritmo no qual as saídas que se encontram dentro das respectivas faixas não são consideradas na função-objetivo do controlador, levando a uma condição de teste de identificação muito próxima a testes em malha aberta. Já o controlador formulado no Problema (P4) e usado neste teste utiliza um conceito novo onde os "set-points" das controladas são variáveis de decisão adicionais calculadas a cada instante de tempo para fornecer o melhor ajuste às saídas enquanto estas estão dentro das respectivas faixas de controle. Com isto, as variáveis controladas nunca estarão "livres", pois mesmo quando dentro das faixas, sempre haverá um erro mínimo considerado na função-objetivo do controlador. 
Uma característica interessante do controlador com a formulação (P4) é justamente a capacidade de reduzir o nível de excitação produzido quando as controladas tendem a se distanciar das faixas. Esta característica, embora possa eventualmente prejudicar a qualidade dos dados gerados para fins de identificação, pode ser interessante numa aplicação real, onde existem outras variáveis de interferência e perturbações que podem, em conjunto com uma excitação fixa e constante, levar a condições de instabilidade na malha de controle.

Concluindo, num controlador com excitação interna como o proposto neste trabalho, sempre será fundamental a análise do engenheiro de controle quanto à qualidade dos dados produzidos e a decisão final da sua utilização ou não para fins de identificação de processos. Exemplos de características dos sinais e/ou do processo a serem consideradas nesta decisão incluem: a variabilidade natural do processo, a presença de perturbações que possam prejudicar o procedimento de identificação, as variâncias dos sinais coletados, o conhecimento das restrições do controlador ativas durante o teste e alguma variável que indique o nível de excitação produzido a cada instante de tempo, entre outros. 


\section{RESULTADOS, CONCLUSÕES E SUGESTÕES DE CONTINUIDADE}

\subsection{CONCLUSÕES}

Este trabalho abordou o problema de reidentificação dos modelos do processo como forma de garantir as especificações de desempenho de controladores preditivos baseados em modelos (MPCs). Os estudos foram desenvolvidos na busca de uma alternativa que propiciasse a geração dos sinais de excitação internamente ao controlador MPC, preservando aspectos de controle e segurança dos processos envolvidos, ou seja, tratando num mesmo problema de otimização requisitos de controle e identificação de sistemas.

Ao término do trabalho, ou pelo menos desta etapa, podem ser listadas as seguintes contribuições principais:

- Foram apresentadas quatro possíveis formulações para inclusão de restrições de excitação num controlador MPC de horizonte finito para fins de identificação, das quais 3 possuem reais perspectivas de aplicação: abordagens (P2), (P3) e (P4). A formulação do Problema (P1) foi de caráter introdutório e prestou-se apenas para avaliar os efeitos da restrição de excitação em estudo no comportamento do MPC.

- Os resultados dos testes realizados e discutidos no capítulo 4 sinalizam que é viável a utilização do controlador com restrição de excitação proposto para fins de identificação de modelos de processos em malha fechada.

- Das formulações estudadas, as que se mostraram mais interessantes foram as abordagens dos Problemas (P3) e (P4), que contornam o problema da não convexidade do Problema (P2) com a execução de dois problemas de otimização a cada instante de tempo. Os problemas $(P 3)$ e $(P 4)$ e garantem que numa 
situação em que o problema com restrição de excitação não seja viável, seja aplicada ao processo a solução do MPC convencional.

- Entre as abordagens dos problemas (P3) e (P4) esta última apresentou melhores características de controle da excitação produzida, o que é desejável do ponto de vista de manutenção das variáveis controladas dentro de limites aceitáveis de afastamento de seus "set-points" ou faixas de controle. O efeito da redução da excitação na qualidade dos dados gerados para fins de identificação deve sempre ser considerado pelo engenheiro de controle.

- Os casos em que se tem a presença de ruídos nos dados devem ser melhor estudados, inclusive com a busca de alternativas para tratamento das amostras e a pesquisa das melhores técnicas de identificação a serem empregadas.

- Entre os inconvenientes observados, o principal diz respeito à estrutura do controlador em todas as formulações estudadas, que não permite a adaptação fácil de controladores normalmente usados na indústria, baseados em problemas de programação linear ou quadrática. As abordagens tratadas neste estudo utilizaram algoritmos de programação quadrática seqüencial (SQP, do inglês "Sequential Quadratic Programming"), cujos tempos de execução são bem maiores que os verificados com o uso de algoritmos LP ou QP.

- Outro ponto a ser considerado com o devido cuidado é quanto ao uso com sistemas de grande porte, que possam exigir matrizes de informação com rank muito elevado. Isto porque, como enfatizado no desenvolvimento das abordagens do capítulo 3, o rank da matriz de informação define diretamente o horizonte de controle mínimo a ser usado $(m>\operatorname{rank}(\Psi))$ e horizontes de controle longos aumentam a carga computacional e o tempo de execução dos controladores MPC. 


\subsection{CONTINUIDADE DOS TRABALHOS}

O assunto da identificação de sistemas em malha fechada, embora bastante estudado e com inúmeras e relevantes contribuições nos últimos anos, ainda apresenta um grande espaço para pesquisa e desenvolvimento, principalmente associado ao uso de controladores MPC. Assim, focando apenas a linha de pesquisa desenvolvida neste trabalho, pode-se apontar as seguintes possibilidades de continuidade:

- Aplicação prática de um controlador com uma das formulações estudadas neste trabalho a um processo real.

- Estudo da influência do rank da matriz de informação (ou da pseudo-matriz de informação) na qualidade ou viabilidade da identificação.

- Estudo do comportamento dos controladores com excitação interna em presença de ruído ou de perturbações não medidas.

- Estudo da relação carga computacional x dimensão do sistema sob identificação, visando avaliar a possibilidade de aplicação das formulações propostas a sistemas reais com elevado número de variáveis.

- Desenvolvimento de critérios de desempenho para controladores MPC que possam apontar a necessidade de ajustes na sintonia ou de reidentificação dos modelos. Tais critérios poderiam inclusive disparar rotinas automáticas de identificação.

- Desenvolvimento de outras formas de excitação interna, que não demandem alteração significativa da estrutura dos controladores. 


\section{REFERÊNCIAS BIBLIOGRÁFICAS}

AGGELOGIANNAKI, E.; SARIMVEIS, H. Multiobjective constrained MPC with simultaneous closed-loop identification. International Journal of Adaptive Control and Signal Processing, v. 20, p. 145-173, 2006.

AGUIRRE, L. A. Introdução à identificação de sistemas: técnicas lineares e nãolineares aplicadas a sistemas reais. $2^{\mathrm{a}}$ ed. Editora UFMG, Belo Horizonte, 2004.

ALMEIDA NETO, E. Controle preditivo multimodelos de uma torre debutanizadora. 1999. 200 p. Dissertação (Mestrado) - Escola Politécnica, Universidade de São Paulo. São Paulo, 1999.

ASTROM, K. J.; WITTENMARK, B. Computer controlled systems: theory and design. $2^{\text {nd }}$ ed. Prentice-Hall, 1990.

CARRAPIÇO, O. L. Controle preditivo de horizonte infinito para processos integradores com tempo morto. 2004. 102 p. Dissertação (Mestrado) - Escola Politécnica, Universidade de São Paulo. São Paulo, 2004.

COLEMAN, T. F.; ZHANG, Y. Optimization toolbox user's guide. Version 3. The Mathworks, Inc., Natick, MA, 2007.

EKER, S. A.; NIKOLAOU, M. Adaptive control through on-line optimization: the MPCI paradigm and variants. International Journal of Applied Mathematics and Computer Science - AMCS, v. 9, n. 1, p. 101-128, 1999.

FORSSELL, U.; LJUNG, L. Closed-loop identification revisited. Automatica, v. 35, n. 7, p. 1215-1241, 1999.

GARCIA, C. Identificação de sistemas. Notas de aula. Universidade de São Paulo, 2005.

GARNIER, H.; GILSON, M.; CERVELLIN, O. Latest developments for the Matlab CONTSID toolbox. In: $14^{\text {th }}$ IFAC SYMPOSIUM ON SYSTEM IDENTIFICATION (SYSID 2006), 2006, Newcastle, Australia. Anais. CD-ROM. 
GENCELI, H.; NIKOLAOU; M. New approach to constrained predictive control with simultaneous model identification. AIChE Journal, v. 42, n. 10, p. 2857-2868, 1996.

GEVERS, M. A personal view of the development of system identification. IEEE Control Systems Magazine, v. 26, n. 6, p. 93-105, 2006.

GONZÁLEZ, A. H.; ODLOAK, D. A stable MPC with zone control. Journal of Process Control, 2008. Artigo aprovado para publicação.

GOODWIN, G. C.; SIN, K. S. Adaptive filtering prediction and control. PrenticeHall, Englewood Cliffs, New Jersey, 1984.

GUSTAVSSON, I. Survey of applications of identification in chemical and physical processes. Automatica, v. 11, n. 1, p. 3-24, 1975.

GUSTAVSSON, I.; LJUNG, L.; SÖDERSTRÖM, T. Identification of processes in closed loop - identifiability and accuracy aspects. Automatica, v. 13, n. 1, p. 59-75, 1977.

LANDAU, I. D. Identification in closed loop: a powerful design tool (better design models, simpler controllers). Control Engineering Practice, v. 9, n. 1, p. 51-65, 2001.

LEE, J. H.; MORARI, M.; GARCIA, C. E. State-space interpretation of model predictive control. Automatica, v. 30, n. 4, p. 707-717, 1994.

LJUNG, L. System identification: theory for the user. $2^{\text {nd }}$ ed. Prentice Hall PTR, New Jersey, 1999.

LJUNG, L. System identification toolbox user's guide. Version 7. The Mathworks, Inc., Natick, MA, 2007.

MACIEJOWSKI, J.M. Predictive Control with Constraints. Prentice Hall, New Jersey, 2002.

MIRANDA, F. C. P. R. Identificação de sistemas em malha fechada usando controlador preditivo multivariável: um caso industrial. 2005. 100 p. Dissertação (Mestrado) - Escola Politécnica, Universidade de São Paulo. São Paulo, 2005. 
MORARI, M.; LEE, J. H. Model predictive control: past, present and future. Computers \& Chemical Engineering, v. 23, p. 667-682, 1999.

ODLOAK, D. A new state space approach to model predictive control. Brazilian Journal of Chemical Engineering, v. 13, n. 3, p. 152-167, 1996.

PORFÍRIO, C. R.; ALMEIDA NETO, E.; ODLOAK, D. Multi-model predictive control of an industrial C3/C4 splitter. Control Engineering Practice, v. 11, n. 7, p. 765-779, 2003.

RIVERA, D. E.; Lee, H.; BRAUN, M. W.; MITTELMANN, H. D. "Plant- friendly" system identification: a challenge for the process industries. In: 13th IFAC SYMPOSIUM ON SYSTEM IDENTIFICATION (SYSID 2003), August, 2003, Rotterdam. Preprints. p. 917-922.

RODRIGUES, M. A.; ODLOAK, D. Stable MPC via dynamic output feedback. In: INTERNATIONAL SYMPOSIUM ON ADVANCED CONTROL OF CHEMICAL PROCESSES (ADCHEM-2000), June, 2000, Pisa, Italy. Preprints.

RODRIGUES, M. A. Estabilidade robusta de controladores preditivos. 2001. 147 p. Tese (Doutorado) - Escola Politécnica, Universidade de São Paulo. São Paulo, 2001.

RODRIGUES, M. A.; ODLOAK, D. An infinite horizon model predictive control for stable and integrating processes. Computers \& Chemical Engineering, v. 27, p. 1113-1128, 2003.

SAGIAS, D.; NIKOLAOU, M. Closed-loop identification of processes operating under model predictive control without external excitation. 2001 AIChE Annual Meeting, paper 279i, Reno - NV, 2001.

SHOUCHE, M. S. Closed-loop identification and predictive control of chemical processes. 1996. $162 \mathrm{p}$. PhD Thesis - Chemical Engineering, Texas A\&M University. 1996.

SHOUCHE, M. S.; GENCELI, H.; NIKOLAOU, M. Effect of on-line optimization techniques on model predictive control and identification (MPCI). Computers \& Chemical Engineering, v. 26, p. 1241-1252, 2002. 
SHOUCHE, M. S.; GENCELI, H.; VUTHANDAM, P.; NIKOLAOU, M. Simultaneous constrained model predictive control and identification of DARX processes.

Automatica, v. 34, n. 12, p. 1521-1530, 1998.

SÖDERSTRÖM, T.; STOICA, P. System identification. Prentice Hall International, Hemel Hempstead, UK, 1989.

SOTOMAYOR, O. A. Z.; ODLOAK, D.; MORO, L. F. L. Closed-loop identification of processes under model predictive control. Control Engineering Practice, 2008. Artigo submetido.

TREIBER, S.; STTELMAIER, R.; STARLING, M. Sustaining benefits of process automation. 2003 AlChE Spring National Meeting, New Orleans, LA, 2003.

TVRZSKÁ DE GOUVÊA, M.; ODLOAK, D. ROSSMPC : A new way of representing and analyzing predictive controllers. Trans/ChemE, v. 75, p. 693-708, 1997.

UNIVERSIDADE DE SÃO PAULO. ESCOLA POLITÉCNICA. DIVISÃO DE BIBLIOTECA. Diretrizes para apresentação de dissertações e teses. São Paulo, 2006.

VAN DEN HOF, P. Closed-loop issues in system identification. Annual Reviews in Control, v. 22, p. 173-186, 1998. 